\title{
Lanthanide tri- and tetrathionates and their complexes with triphenylphosphine oxide
}

Simon J Coles ${ }^{1}$, Sarah J. Fieldhouse ${ }^{2}$, Wim T. Klooster ${ }^{1}$, Anthony M.J.Lees ${ }^{2}$ and Andrew W. G. Platt $^{2 *}$

1. UK National Crystallography Service, Chemistry, University of Southampton, Highfield Campus, Southampton, SO17 1BJ, UK

2. School of Law Policing and Forensic Science, Staffordshire University, Leek Road, Stoke-onTrent, ST4 2DF, UK

doi.org/10.1016/j.poly.2020.114404

\section{Abstract}

Lanthanide tri- and tetrathionate containing species have been prepared in solution by the reaction of potassium tri- and tetrathionate with lanthanide perchlorates. Unstable solid materials from these reactions have been examined by infrared spectroscopy and electrospray mass spectrometry (ESI MS). There was evidence of the formation of $\mathrm{S}_{3} \mathrm{O}_{6}{ }^{2-}$ and $\mathrm{S}_{4} \mathrm{O}_{6}{ }^{2-}$ coordination to lanthanide ions, but it was not possible to produce material suitable for single crystal x-ray studies. The reaction of ethanol solutions from $\mathrm{Ln}\left(\mathrm{ClO}_{4}\right)_{3} / \mathrm{K}_{2} \mathrm{~S}_{\mathrm{n}} \mathrm{O}_{6}(\mathrm{n}=3,4)$ with triphenylphosphine oxide yielded crystalline materials which have been characterised by infrared spectroscopy, electrospray mass spectrometry, elemental analysis and single crystal X-ray diffraction. The trithionate complexes $\left[\mathrm{Ln}\left(\mathrm{S}_{3} \mathrm{O}_{6}\right)\left(\mathrm{Ph}_{3} \mathrm{PO}\right)_{4}\right] \mathrm{ClO}_{4} .3 \mathrm{H}_{2} \mathrm{O}(\mathrm{Ln}=\mathrm{Nd}, \mathrm{Tb})$ have been isolated. The tetrathionate / $\mathrm{Ph}_{3} \mathrm{PO}$ reaction gave rise to significant disproportionation giving a small quantity of $\left[\mathrm{Nd}\left(\mathrm{S}_{4} \mathrm{O}_{6}\right)\left(\mathrm{Ph}_{3} \mathrm{PO}\right)_{4}\left(\mathrm{H}_{2} \mathrm{O}\right)\right] \mathrm{ClO}_{4}$, a different isomer of $\left[\mathrm{Nd}\left(\mathrm{S}_{3} \mathrm{O}_{6}\right)\left(\mathrm{Ph}_{3} \mathrm{PO}\right)_{4} \mathrm{H}_{2} \mathrm{O}\right] \mathrm{ClO}_{4}$, neodymium sulfate and elemental sulfur. The crystal structures of the two isomers of $\left[\mathrm{Nd}\left(\mathrm{S}_{3} \mathrm{O}_{6}\right)\left(\mathrm{Ph}_{3} \mathrm{PO}\right)_{4} \mathrm{H}_{2} \mathrm{O}\right] \mathrm{ClO}_{4}$ and that of $\left[\mathrm{Nd}\left(\mathrm{S}_{4} \mathrm{O}_{6}\right)\left(\mathrm{Ph}_{3} \mathrm{PO}\right)_{4} \mathrm{H}_{2} \mathrm{O}\right] \mathrm{ClO}_{4}$ are reported. The structure of $\left[\mathrm{Er}\left(\mathrm{H}_{2} \mathrm{O}\right)_{5}\left(\mathrm{Ph}_{3} \mathrm{PO}\right)_{2}\right] .5 \mathrm{Ph}_{3} \mathrm{PO} .3\left[\mathrm{ClO}_{4}^{-}\right]$, formed during the attempted preparation of erbium trithionate complexes, is also described.

\section{Introduction}

Metal complexes involving di-, tri- and tetrathionate ions, $\left(\mathrm{S}_{\mathrm{n}} \mathrm{O}_{6}{ }^{2-}, \mathrm{n}=2,3,4\right)$, are largely limited to their role as anions in cationic complexes. There are a considerable number of compounds 
containing dithionate in which the $\left[\mathrm{S}_{2} \mathrm{O}_{6}\right]^{2-}$ ion acts as an uncoordinated counterion. The coordinated ion occurs less frequently, but has been reported as a monodentate ligand with copper(II) [1-4] and zinc(II) [5] in complexes with N-containing macrocycles. Bridging dithionate has also been reported, and is found mainly in the $\kappa^{2}-O O^{\prime}$ mode [3, 6-9] but also as bridging $\kappa^{2}-O^{2}$ [10], again coordinated to $\mathrm{Cu}(\mathrm{II})$. Structures where a higher polythionate ion is coordinated to a metal are limited to bridging $\mathrm{S}_{3} \mathrm{O}_{6}{ }^{2-}$ in $[\mathrm{Na}(18-\mathrm{C}-6)]_{2} \mathrm{~S}_{3} \mathrm{O}_{6}[11]$ and $\left[\mathrm{Cu}\left(\mathrm{S}_{3} \mathrm{O}_{6}\right)\left(\mathrm{H}_{2} \mathrm{O}\right)_{4}\right]_{n}[12]$, bridging $\mathrm{S}_{4} \mathrm{O}_{6}{ }^{2-}$ in $\mathrm{Cu}(1,10-\text { phen })_{2} \mathrm{~S}_{4} \mathrm{O}_{6}$ [13], and bidentate $\mathrm{S}_{4} \mathrm{O}_{6}{ }^{2-}$ in $\mathrm{Mn}(\text { bipy })_{2} \mathrm{~S}_{4} \mathrm{O}_{6}$ [14] and in $\mathrm{M}(4,4 \text { ' dimethyl-2,2'-bipy })_{2} \mathrm{~S}_{4} \mathrm{O}_{6} \mathrm{M}=\mathrm{Zn}, \mathrm{Cd}[15]$.

We have previously reported the preparation and structures of some lanthanide dithionates which gave a polymeric structure for $\mathrm{Nd}_{2}\left(\mathrm{~S}_{2} \mathrm{O}_{6}\right)_{3} \cdot 14 \mathrm{H}_{2} \mathrm{O}$ and their triphenylphosphine oxide complexes $\mathrm{Nd}_{2}\left(\mathrm{~S}_{2} \mathrm{O}_{6}\right)_{3}\left(\mathrm{Ph}_{3} \mathrm{PO}\right)_{4} \cdot 8 \mathrm{H}_{2} \mathrm{O}[16]$ and $\mathrm{Pr}_{2}\left(\mathrm{~S}_{2} \mathrm{O}_{6}\right)_{3}\left(\mathrm{Ph}_{3} \mathrm{PO}\right)_{6} \cdot 6 \mathrm{H}_{2} \mathrm{O}$ [17]. Other than these dithionates, there are no reports of lanthanide polythionates $\left({ }^{-} \mathrm{O}_{3} \mathrm{~S}(\mathrm{~S})_{n} \mathrm{SO}_{3}{ }^{-}\right)$or their coordination complexes, and we report here our investigation into these under-represented compounds with the aim of obtaining materials suitable for structural determination.

\section{Results and discussion}

\subsection{Attempted preparation of lanthanide trithionates and tetrathionates}

The preparation of lanthanide trithionates and tetrathionates was attempted by metathesis between $\mathrm{Ln}\left(\mathrm{ClO}_{4}\right)_{3}(\mathrm{Ln}=\mathrm{La}, \mathrm{Pr}, \mathrm{Nd}, \mathrm{Tb}, \mathrm{Er}, \mathrm{Yb})$ and $\mathrm{K}_{2} \mathrm{~S}_{\mathrm{n}} \mathrm{O}_{6}$ in aqueous solution followed by precipitation of the potassium salts with ethanol in which the lanthanide polythionate species appeared soluble. On addition of ethanol, the ethanolic solutions retained the characteristic colour of the lanthanide ion $(\mathrm{Pr}$ and $\mathrm{Nd}$ ) while precipitated material was white, indicating that it contained little or no insoluble lanthanide compounds. Attempted crystallisation of these solutions by fractional crystallisation led to the isolation of small quantities of materials which were analysed by infrared spectroscopy. The infrared spectra showed, in addition to peaks due to coordinated water at 3300 and $1630 \mathrm{~cm}^{-1}$ respectively, strong absorptions due to $\mathrm{SO}_{3}$ or $\mathrm{ClO}_{4}^{-}$groups between $1110-1080 \mathrm{~cm}^{-}$

${ }^{1}, 1010-1005 \mathrm{~cm}^{-1}, 930-920 \mathrm{~cm}^{-1}$ and $835-830 \mathrm{~cm}^{-1}$ for the trithionates and 1220, 1180, 1050 and 
$1010 \mathrm{~cm}^{-1}$ for the tetrathionates. These absorption bands are shifted to lower wavenumber in comparison to those observed in $\mathrm{Na}_{2} \mathrm{~S}_{3} \mathrm{O}_{6}$ and $\mathrm{K}_{2} \mathrm{~S}_{4} \mathrm{O}_{6}$ (which have strong bands at 1251,1213 , $1066,1024 \mathrm{~cm}^{-1}$ and $1255,1224,1195,1037$ and $1012 \mathrm{~cm}^{-1}$ respectively), indicating that both $\mathrm{S}_{3} \mathrm{O}_{6}{ }^{2-}$ and $\mathrm{S}_{4} \mathrm{O}_{6}{ }^{2-}$ are coordinated to the lanthanide ion.

Reactions with heavier lanthanides led to the isolation of materials whose infrared spectra indicated that they contained significant amounts of sulfate [18]. We attribute this to the decomposition of both trithionate and tetrathionate in mildly acidic aqueous solution, which is known to produce thiosulfate and sulfate [19].

Electrospray mass spectra were obtained from methanol solutions of the materials obtained from the Pr and Nd reactions. These ESI-MS spectra confirm the presence of both lanthanide ion and polythionate in the isolated materials. Details of the observed ions and their assignments are given in Table S1. The spectra show that $\left[\mathrm{LnO}\left(\mathrm{H}_{2} \mathrm{O}\right)_{\mathrm{n}}\right]^{+}$and $\left[\mathrm{LnO}(\mathrm{MeOH})_{\mathrm{n}}\right]^{+}$are the most abundant ions in the positive ion mode. This behaviour is typical of the behaviour of lanthanide complexes with weakly coordinating anions under electrospray conditions [20]. There are a series of low abundance ions containing the trithionate and tetrathionate ions corresponding to $\left[\mathrm{Ln}\left(\mathrm{S}_{3} \mathrm{O}_{6}\right)(\mathrm{MeOH})_{\mathrm{m}}\left(\mathrm{H}_{2} \mathrm{O}\right)_{\mathrm{n}}\right]^{+}(\mathrm{m}=0-2, \mathrm{n}=1-4)$ and $\left[\mathrm{Ln}\left(\mathrm{S}_{4} \mathrm{O}_{6}\right)\left(\mathrm{H}_{2} \mathrm{O}\right)_{2}\right]^{+} \mathrm{Ln}=\mathrm{Nd}$. Similarly the negative ion spectra (Table S2) show peaks associated with the trithionate and tetrathionate ions $\left[\mathrm{S}_{3} \mathrm{O}_{6}(\mathrm{MeOH})_{\mathrm{n}}\right]^{2-}$ and $\left[\mathrm{S}_{4} \mathrm{O}_{6} \mathrm{H}\right]^{-}$as the most abundant ions in the spectra, with lower abundance ions from $\left[\mathrm{Ln}\left(\mathrm{S}_{3} \mathrm{O}_{6}\right)_{2}(\mathrm{MeOH})_{\mathrm{m}}\right]^{-},\left[\mathrm{Ln}\left(\mathrm{S}_{3} \mathrm{O}_{6}\right)_{2}\left(\mathrm{H}_{2} \mathrm{O}\right)_{\mathrm{n}}\right]^{-}(\mathrm{Ln}=\mathrm{Pr}, \mathrm{Nd})$ and $\left[\mathrm{Nd}\left(\mathrm{S}_{4} \mathrm{O}_{6}\right)_{2}\right]^{-}$and $\left[\mathrm{ClO}_{4}\right]^{-}$. Assignments are based on the calculated $\mathrm{m} / \mathrm{z}$ values and comparison of theoretical and observed isotope profiles An example is shown in Figure 1 for the $\left[\mathrm{Nd}\left(\mathrm{S}_{3} \mathrm{O}_{6}\right)_{2}\right]^{-}$ion. 

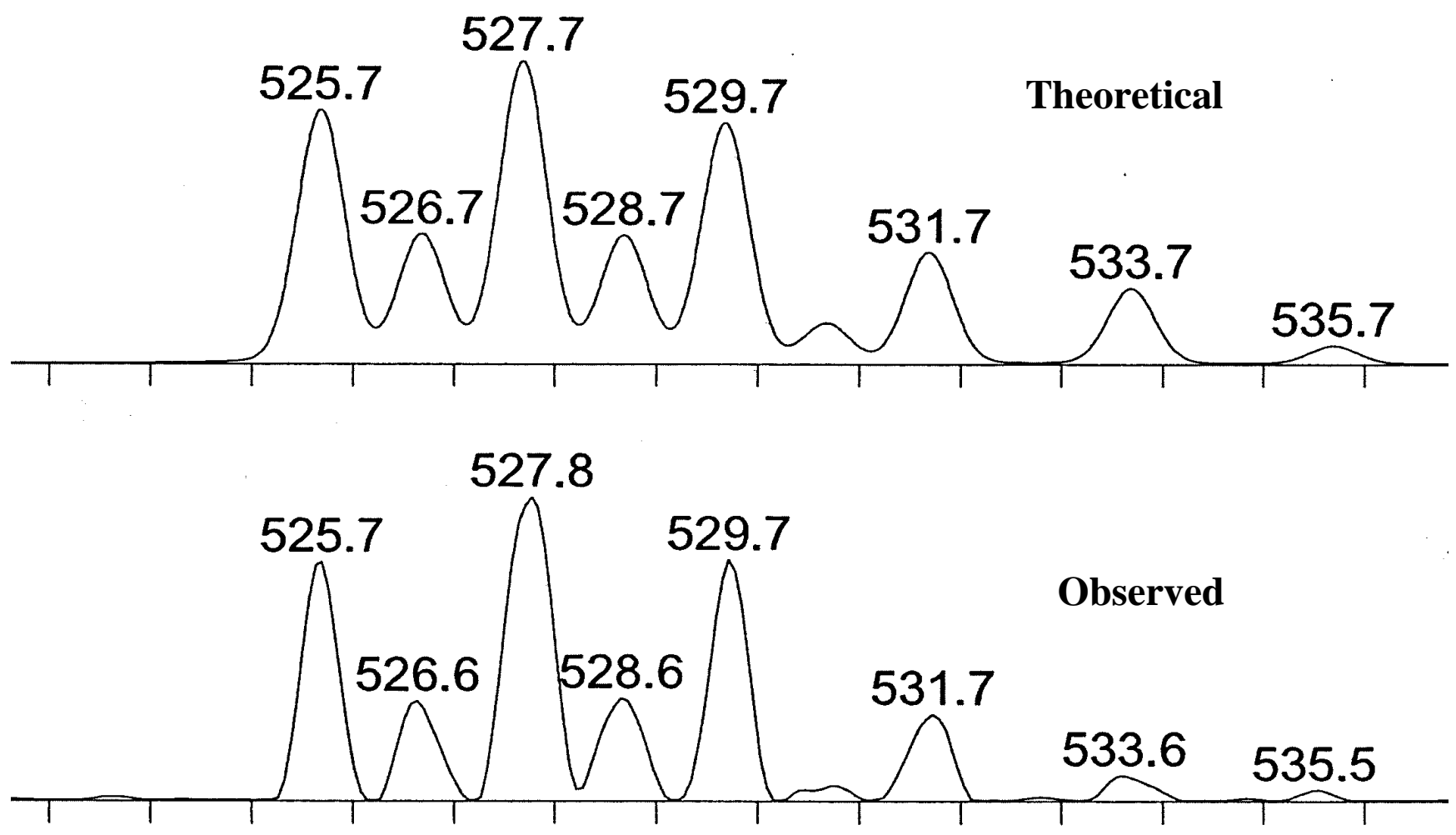

Figure 1 Theoretical and Observed Isotope Profiles for $\left[\mathrm{Nd}\left(\mathrm{S}_{3} \mathrm{O}_{6}\right)_{2}\right]^{2-}$

Unfortunately, unlike the dithionates previously reported [16], and despite many attempts, it has proved impossible to produce crystalline material suitable for single crystal X-ray studies. From the available evidence (and see also below) it seems that full replacement of perchlorate by the polythionate ions has not occurred and the species produced are tentatively assigned as “[ $\left[\mathrm{Ln}\left(\mathrm{S}_{\mathrm{n}} \mathrm{O}_{6}\right)\left(\mathrm{ClO}_{4}\right)\right] \cdot \mathrm{xH}_{2} \mathrm{O} "$.

\subsection{Coordination complexes of lanthanide trithionates with triphenylphosphine oxide}

In view of the lack of success in preparing stable tri- and tetrathionates we attempted the in situ synthesis of coordination complexes in the hope that these might be more stable and give crystalline materials suitable for X-ray diffraction studies. Triphenylphosphine oxide often produces crystalline complexes with organic materials [21] and with lanthanide salts [22]. Thus, complex formation with $\mathrm{Ph}_{3} \mathrm{PO}$ seemed a potential route to the synthesis of stable compounds which could be characterised by X-ray crystallography. 
Ethanolic solutions of lanthanide trithionate species from the reaction of lanthanide perchlorates and the potassium trithionate, described above, were added to solutions of triphenylphosphine oxide. The bulk materials isolated by fractional crystallisation of the solutions gave elemental analyses which indicated that $\left[\mathrm{Ln}\left(\mathrm{S}_{3} \mathrm{O}_{6}\right)\left(\mathrm{Ph}_{3} \mathrm{PO}\right)_{4}\left(\mathrm{H}_{2} \mathrm{O}\right)\right] \mathrm{ClO}_{4} \cdot 2 \mathrm{H}_{2} \mathrm{O}$ could be produced up to $\mathrm{Ln}=$ Tb.

The infrared spectra of these materials are consistent with the formation of coordination complexes. The IR spectra show bands due to coordinated water,the $\mathrm{P}=\mathrm{O}$ stretch at around 1140 $\mathrm{cm}^{-1}$, and a complex series of intense absorptions at around 1290, 1090, 1040 and $660 \mathrm{~cm}^{-1}$ due to $\mathrm{S}-\mathrm{O}$ and $\mathrm{Cl}-\mathrm{O}$ stretches. The $\mathrm{P}=\mathrm{O}$ stretch is significantly lower than observed in free $\mathrm{Ph}_{3} \mathrm{PO}(1190$ $\left.\mathrm{cm}^{-1}\right)$.

The isolated complexes are poorly soluble in most common organic solvents and it has not been possible to obtain NMR spectra in any organic solvent but methanol. The ${ }^{31} \mathrm{P}$ NMR spectra of methanolic solutions of the complexes showed a single signal close to that of free $\mathrm{Ph}_{3} \mathrm{PO}$. The absence of significant paramagnetic shifts indicates that in this solvent extensive dissociation occurs on dissolution.

Fractional crystallisation of the original ethanol solution of the $\mathrm{Nd}$ complex gave crystals suitable for X-ray diffraction studies. Full descriptions of the data collection and refinement are given in the supplementary information, as are figures of the structures with thermal ellipsoids at $50 \%$ probability together with full listings of bond distances and angles. The crystalline material was shown to be aqua trithionato- $\kappa^{4} O, O, O^{\prime}, O^{\prime}$ tetrakis-triphenylphosphine oxide neodymium(III) perchlorate. The cation, $\left.\left[\mathrm{Nd}\left(\mathrm{S}_{3} \mathrm{O}_{6}\right)\left(\mathrm{Ph}_{3} \mathrm{PO}\right)_{4}\right)\left(\mathrm{H}_{2} \mathrm{O}\right)\right]^{+}$, is 9-coordinate:the neodymium ion is bound to the 4 oxygen atoms from the $\mathrm{Ph}_{3} \mathrm{PO}$ ligands, the oxygen atom of the water molecule, and 4 oxygen atoms from the trithionate ion (two from each $\mathrm{SO}_{3}$ group),

Table 1 Selected bond distances ${ }^{\mathrm{a}}(\AA)$ in $\left[\mathrm{Nd}\left(\mathrm{S}_{3} \mathrm{O}_{6}\right)\left(\mathrm{Ph}_{3} \mathrm{PO}\right)_{4}\left(\mathrm{H}_{2} \mathrm{O}\right)\right]^{+}$

\begin{tabular}{|c|c|c|c|c|c|}
\hline \multirow[t]{2}{*}{ Bond } & \multicolumn{2}{|c|}{$\left.\mathrm{Nd}\left(\mathrm{S}_{3} \mathrm{O}_{6}\right)\left(\mathrm{Ph}_{3} \mathrm{PO}\right)_{4}\left(\mathrm{H}_{2} \mathrm{O}\right)\right]^{+}$} & $\mathrm{K}_{2} \mathrm{~S}_{3} \mathrm{O}_{6}{ }^{\mathrm{b}}$ & $(\mathrm{Na}-18 \mathrm{C}-6)_{2}-$ & {$\left[\mathrm{Cu}\left(\mathrm{H}_{2} \mathrm{O}\right)_{4} \mu \mathrm{S}_{3} \mathrm{O}_{6}\right]_{\mathrm{n}}{ }^{\mathrm{d}}$} \\
\hline & $K^{4}-O, O, O^{\prime}, O^{\prime}$ & $K^{3}-O, O, O^{\prime \mathrm{e}}$ & & & \\
\hline $\mathrm{O}_{3} \mathrm{~S}-\mathrm{S}$ & $2.10(2)$ & $2.11(4)$ & $2.083(22)$ & $2.111(8)$ & $2.132(18)$ \\
\hline
\end{tabular}




\begin{tabular}{|c|c|c|l|l|l|}
\hline & & & & & \\
\hline $\mathrm{S}-\mathrm{O}(\mathrm{M})$ & $1.50(9)$ & $1.46(2)$ & & $1.445(2)$ & $1.452(3)$ \\
\hline $\mathrm{S}=\mathrm{O}$ & $1.43(3)$ & $1.40(3)$ & $1.450(6)$ & $1.440(4)$ & $1.450(4)$ \\
\hline $\mathrm{Nd}-\mathrm{O}(\mathrm{S})$ & $2.59(11)$ & $2.59(15)$ & & & \\
\hline $\mathrm{Nd}-\mathrm{O}(\mathrm{P})$ & $2.334(18)$ & $2.36(1)$ & & & \\
\hline $\mathrm{P}=\mathrm{O}$ & $1.504(1)$ & $1.504(5)$ & & & \\
\hline $\mathrm{Nd}-\mathrm{O}\left(\mathrm{H}_{2} \mathrm{O}\right)$ & $2.65(13)$ & $2.51(4)$ & & & \\
\hline & & & & & \\
\hline
\end{tabular}

a. Values in parenthesis are standard deviations which reflect the variation in bond distances rather than uncertainties in the data collection. b. data from ref 23 c. data from ref 14. d. data from ref 15 e. see below

The uncoordinated perchlorate ion is hydrogen bonded to the coordinated water by two oxygen atoms, the O..... distances are 2.856 and $2.783 \AA$. The bond distances are summarised in Table 1 and compared with those of $\mathrm{K}_{2} \mathrm{~S}_{3} \mathrm{O}_{6}$ [23], the $\kappa^{3}$ complex discussed in section 2.3, and two other published trithionato-complexes. The $\mathrm{S}_{-} \mathrm{SO}_{3}$ distances do not differ significantly from the previously published structures, but the $\mathrm{S}=\mathrm{O}$ bond is slightly shorter in the $\mathrm{Nd}$ complex. The $\mathrm{S}$ $\mathrm{O}(\mathrm{Nd})$ distance is significantly longer than the $\mathrm{S}=\mathrm{O}$ length as expected on coordination to the more highly charged lanthanide ion. The geometry of the cation was analysed using the SHAPE programme which locates the idealised structure which best conforms to the observed geometry $[22,23]$. In this case two geometries give good descriptions of the structure, a 1,5,3 "muffin", and a capped square antiprism [24]. Both representations of the structure are shown in Figure 2.

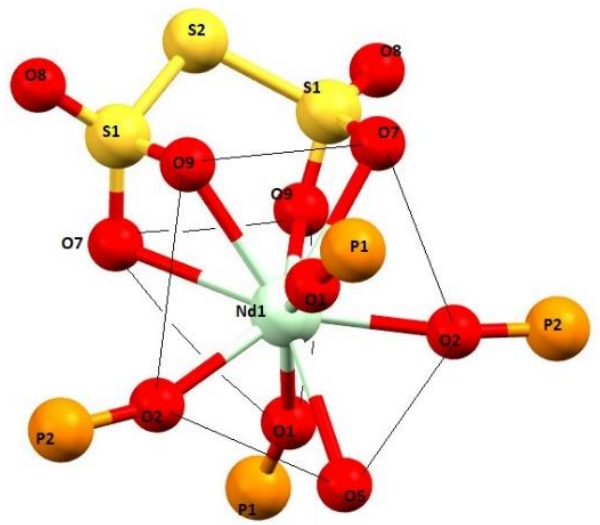

A

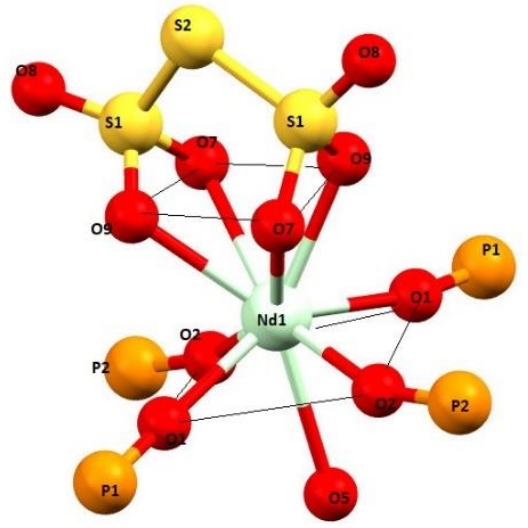

B 
Figure 2 The core structures of A. the "muffin" and B. the capped square antiprism views of $\left[\kappa^{4}-O, O, O^{\prime}, O^{\prime}-\mathrm{Nd}\left(\mathrm{S}_{3} \mathrm{O}_{6}\right)\left(\mathrm{H}_{2} \mathrm{O}\right)\left(\mathrm{Ph}_{3} \mathrm{PO}\right)_{4}\right]^{+}$. Hydrogen atoms, phenyl groups and perchlorate ions omitted for clarity.

In the 1,5,3 "muffin" geometry $\mathrm{O} 1$ is the 1 - atom, the 5-plane is defined by $\mathrm{O} 2, \mathrm{O} 2, \mathrm{O} 5, \mathrm{O} 7$ and $\mathrm{O} 9$ and the 3-plane by $\mathrm{O} 1, \mathrm{O} 7$ and $\mathrm{O} 9$. The angle between the 5- and 3-planes is $14.3^{\circ}$ and the deviation of the oxygen atoms in the 5-plane ranges between $0.006-0.320 \AA$. In the square antiprismatic representation the square planes are defined by the $\mathrm{O} 1, \mathrm{O} 1, \mathrm{O} 2, \mathrm{O} 2$ from the $\mathrm{Ph}_{3} \mathrm{PO}$ and the $\mathrm{O} 7,07, \mathrm{O} 9$ and $\mathrm{O} 9$ atoms from the trithionate. The planes are parallel with the deviation of the oxygen atoms from the mean planes of $0.052 \AA$ and $0.114 \AA$ respectively.

The Nd and three sulfur atoms are coplanar with the coordinated oxygen atoms lying on average $1.03 \AA$ above and below this plane, such that the 6-membered ring adopts a chair configuration.

Attempted preparation of the erbium trithionate - triphenylphosphine oxide complex led to a solid whose elemental analysis did not correspond to any simple trithionate complex. The absence of strong S-O stretches at around $1290 \mathrm{~cm}^{-1}$ in the infrared spectrum of the isolated solid indicated that the trithionate ion was not present. Small amounts of crystalline material were obtained which single crystal x-ray diffraction showed to be $\left[\mathrm{Er}\left(\mathrm{H}_{2} \mathrm{O}\right)_{5}\left(\mathrm{Ph}_{3} \mathrm{PO}\right)_{2}\right] \cdot\left(\mathrm{Ph}_{3} \mathrm{PO}\right)_{5} \cdot 3\left[\mathrm{ClO}_{4}\right]$. The structure is shown in Figure 3. The erbium ion is 7-coordinate with a pentagonal bipyramidal geometry made up by 5 water molecules in the equatorial plane and 2 axial $\mathrm{Ph}_{3} \mathrm{PO}$ in the first coordination sphere. The remaining $5 \mathrm{Ph}_{3} \mathrm{PO}$ molecules are $\mathrm{H}$-bonded to the coordinated waters. The structure is similar to those of $\left[\mathrm{Ln}\left(\mathrm{H}_{2} \mathrm{O}\right)_{5}\left(\mathrm{Cy}{ }_{3} \mathrm{PO}\right)_{2}\right] \cdot 2 \mathrm{Cy}_{3} \mathrm{PO} \cdot \mathrm{X}_{3} \mathrm{X}=\mathrm{CF}_{3} \mathrm{SO}_{3}[25], \mathrm{Cl}[26,27]$ and $\mathrm{Br}[26,28]$. The coordinated water molecules lie within $0.06 \AA$ of the mean O8, O9, O10, O11, O12 plane. This is the first time this type of complex has been reported with a relatively smaller and lower basicity triaryl phosphine oxide. The structure is unusual in that $5 \mathrm{Ph}_{3} \mathrm{PO}$ are hydrogen bonded and presumably this is reflection of the lower steric demands of $\mathrm{Ph}_{3} \mathrm{PO}$ compared to $\mathrm{Cy}_{3} \mathrm{PO}$. 


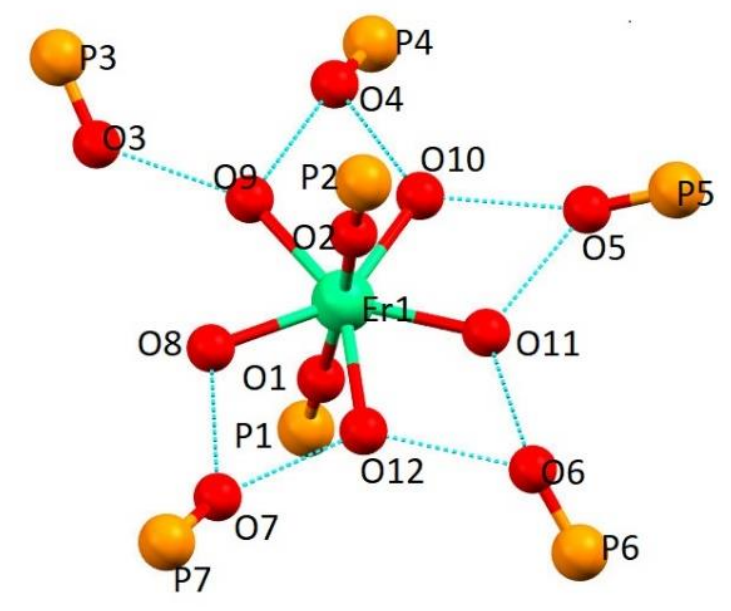

Figure 3 The core structure of the $\left[\mathrm{Er}\left(\mathrm{H}_{2} \mathrm{O}\right)_{5}\left(\mathrm{Ph}_{3} \mathrm{PO}\right)_{2} \cdot\left(\mathrm{Ph}_{3} \mathrm{PO}\right)_{5}\right]^{3+}$ cation. Blue broken linies represent hydrogen bonds. Hydrogen atoms, phenyl groups and perchlorate ions omitted for clarity

\subsection{Coordination complexes of lanthanide tetrathionates with triphenylphosphine oxide}

Attempted preparation of triphenylphosphine oxide complexes of tetrathionates by the same method as the trithionates led to mixtures of compounds. The reaction of triphenylphosphine oxide with the ethanolic solutions obtained from the mixing of potassium tetrathionate and neodymium perchlorate was studied in detail. Four different solid products were identified from slow evaporation of the reaction mixture and a sample was mechanically separated into individual components. A small quantity of crystals formed at the air solution interface during slow evaporation of the solution which single crystal X-ray diffraction showed to be aqua tetrathionato$\kappa^{2}-O, O^{\prime}$-tetrakis-triphenylphosphine oxide neodymium(III) perchlorate, $\left[\mathrm{Nd}\left(\mathrm{S}_{4} \mathrm{O}_{6}\right)\left(\mathrm{Ph}_{3} \mathrm{PO}\right)_{4}\left(\mathrm{H}_{2} \mathrm{O}\right)\right] \mathrm{ClO}_{4}$. Electrospray mass spectrometry of this complex in methanol shows that extensive dissociation and ligand redistribution occurs under the experimental 
conditions. Details of the ions identified are given in supplementary material in Table S3.

Filtration of the reduced solution produced larger quantities of a different crystalline complex, elemental sulfur and a lilac powder. The infrared spectrum of the crystalline material was very similar to that of the trithionate complex reported in section 2.2 and showed only slight differences in the positions of absorption maxima and their relative intensities. (See Figure S1 in the supplementary information). On analysis by single crystal x-ray diffraction this proved to be aqua trithionato- $\kappa^{3} O, O, O^{\prime}$-tetrakis-triphenylphosphine oxide neodymium(III) perchlorate, $\left[\mathrm{Nd}\left(\mathrm{S}_{3} \mathrm{O}_{6}\right)\left(\mathrm{Ph}_{3} \mathrm{PO}\right)_{4}\left(\mathrm{H}_{2} \mathrm{O}\right)\right] \mathrm{ClO}_{4}$ (discussed below). The presence of elemental sulfur was confirmed by gas chromatography - mass spectrometry of a carbon disulfide solution which had an identical retention time and mass spectrum to that of an authentic sample of sulfur, $\mathrm{m} / \mathrm{z} 256$ with an isotope profile matching that of $\mathrm{S}_{8}$. The lilac powder had an infrared spectrum which showed the presence of coordinated water but no triphenylphosphine oxide, and no bands in the $1200 \mathrm{~cm}^{-1}$ region which could be associated with tri- or tetrathionate. Instead a series of 4 intense bands at 1101, 996, 934 and $752 \mathrm{~cm}^{-1}$ were observed. The presence of $4 \mathrm{~S}-\mathrm{O}$ stretches is expected for bridging sulfate and the spectra were similar to those reported for $\operatorname{Ln}_{2}\left(\mathrm{SO}_{4}\right)_{3} \cdot 8 \mathrm{H}_{2} \mathrm{O}[31,32]$. The presence of trithionate, elemental sulfur and sulfate indicates that a complex disproportionation reaction or series of reactions is occurring. The decomposition of tetrathionate to trithionate and thiosulfate under a variety of conditions has been reported [19] and the thiosulfate produced in these reactions could further decompose in mildly acidic solution to elemental sulfur and sulfur dioxide, although the latter was not detected in our system.

The cation in $\left[\mathrm{Nd}\left(\mathrm{S}_{4} \mathrm{O}_{6}\right)\left(\mathrm{H}_{2} \mathrm{O}\right)\left(\mathrm{Ph}_{3} \mathrm{PO}\right)_{4}\right] \mathrm{ClO}_{4}$ is 7 -coordinate: the central $\mathrm{Nd}$ ion is bound to 2 oxygen atoms of the tetrathionate ligand (one from each $\mathrm{SO}_{3}$ group), 4 oxygen atoms from the $\mathrm{Ph}_{3} \mathrm{PO}$ and the oxygen atom of the water molecule. Analysis of the geometry of the cation, as above, gives a bicapped trigonal prism as the best fit to common 7-coordinate structures [33]. The trigonal faces are defined by the $\mathrm{O} 1, \mathrm{O} 2$, and $\mathrm{O} 4$ atoms from the two phoshine oxides and the tetrathionate respectively. The angle between the triangular faces is $5.03^{\circ}$. The water oxygen atom, 
$\mathrm{O} 3$, caps the $\mathrm{O} 1, \mathrm{O} 1, \mathrm{O} 2, \mathrm{O} 2$ square face. The angle between the square faces ranges between 68.3

to $55.9^{\circ}$ with an average of $60^{\circ}$. The core coordination in the complex cation is shown in Figure 4.

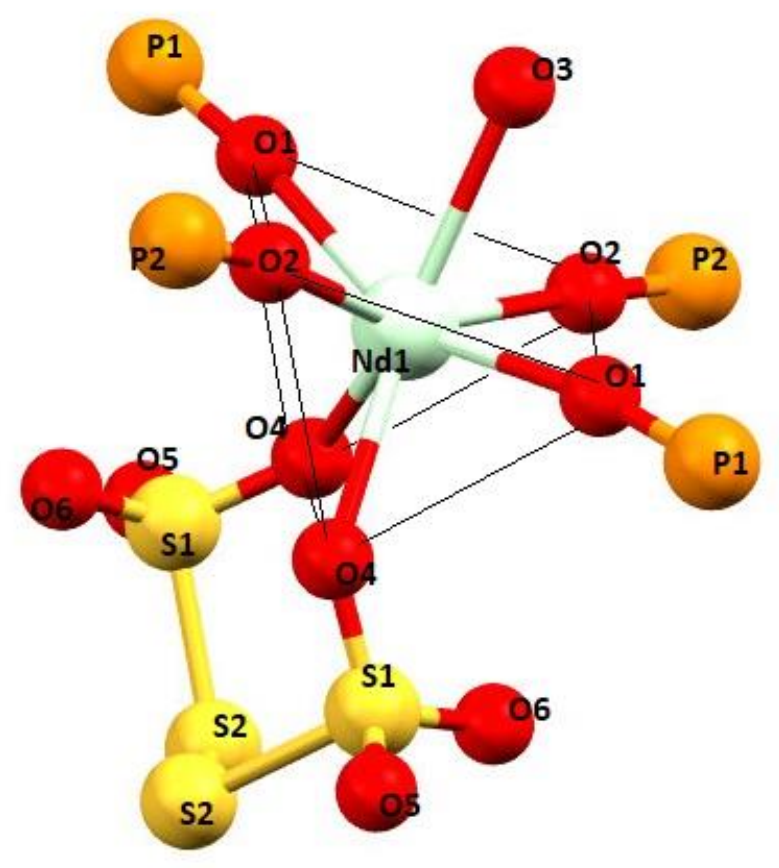

Figure 4 The core structure of the capped trigonal prismatic $\left[\mathrm{Nd}\left(\mathrm{S}_{4} \mathrm{O}_{6}\right)\left(\mathrm{H}_{2} \mathrm{O}\right)\left(\mathrm{Ph}_{3} \mathrm{PO}\right)_{4}\right]^{+}$. Hydrogen atoms, phenyl groups and perchlorate ions omitted for clarity.

In a similar manner to the trithionate analogue, the perchlorate ion is hydrogen bonded via two oxygen atoms $(\mathrm{O} \ldots \mathrm{O} 2.868 \AA$ ) and this is reflected in a slight lengthening of the $\mathrm{Cl}-\mathrm{O}$ bonds (1.434 compared to $1.399 \AA$ ). The bond distances are summarised in Table 2 and compared with those found in $\mathrm{K}_{2} \mathrm{~S}_{4} \mathrm{O}_{6}$ [34] and complexes where the tetrathionate ion acts as a chelate [14, 15]. As observed for the trithionate complex, the $\mathrm{S}_{-} \mathrm{SO}_{3}$ bond distances are little affected by coordination to the neodymium compared to the tetrathionate ion in $\mathrm{K}_{2} \mathrm{~S}_{4} \mathrm{O}_{6}$ but are marginally longer than in complexes with divalent metal ions. The bond distances within the coordinated tetrathionate ion are little affected by binding to the metal in the Nd complex and those with Mn [14], $\mathrm{Zn}$ and $\mathrm{Cd}[15]$. The exception to this is the $\mathrm{S}-\mathrm{O}(\mathrm{M})$ length which is significantly longer due to coordination to the trivalent $\mathrm{Nd}$ centre.

Table 2 Selected bond distances $(\AA)$ and $\operatorname{angles}\left({ }^{\circ}\right)$ in $\left[k^{2}-O, O^{\prime} \mathrm{Nd}\left(\mathrm{S}_{4} \mathrm{O}_{6}\right)\left(\mathrm{H}_{2} \mathrm{O}\right)\left(\mathrm{Ph}_{3} \mathrm{PO}\right)_{4}\right]^{+}$ compared with known structures

\begin{tabular}{|l|l|l|l|l|l|}
\hline & {$\left[\mathrm{Nd}\left(\mathrm{S}_{4} \mathrm{O}_{6}\right)\left(\mathrm{H}_{2} \mathrm{O}\right)\left(\mathrm{Ph}_{3} \mathrm{PO}\right)_{4}\right]^{+}$} & $\mathrm{K}_{2} \mathrm{~S}_{4} \mathrm{O}_{6}{ }^{\mathrm{b}}$ & $\mathrm{Mn}\left(\mathrm{S}_{4} \mathrm{O}_{6}\right)(\text { bipy })_{2}{ }^{\mathrm{c}}$ & $\mathrm{Zn}\left(\mathrm{S}_{4} \mathrm{O}_{6}\right) \mathrm{L}_{2}{ }^{\mathrm{d}}$ & $\mathrm{Cd}_{\left(\mathrm{S}_{4} \mathrm{O}_{6}\right) \mathrm{L}_{2}{ }^{\mathrm{d}}}$ \\
\hline
\end{tabular}




\begin{tabular}{|l|l|l|l|l|l|}
\hline $\mathrm{O}_{3} \mathrm{~S}-\mathrm{S}$ & $2.115(3)^{\mathrm{a}}$ & 2.117 & 2.066 & 2.106 & 2.101 \\
\hline $\mathrm{S}-\mathrm{S}$ & $2.011(4)$ & 2.017 & 1.963 & 2.016 & 2.017 \\
\hline $\mathrm{S}-\mathrm{O}(\mathrm{M})$ & $1.477(2)$ & 1.446 & 1.446 & 1.441 & 1.430 \\
\hline $\mathrm{S}=\mathrm{O}$ & $1.425(16)$ & 1.446 & 1.410 & 1.421 & 1.411 \\
\hline $\mathrm{M}-\mathrm{O}(\mathrm{S})$ & $2.431(20)$ & 2.89 & 2.122 & 2.277 & 2.120 \\
\hline $\mathrm{M}-\mathrm{O}(\mathrm{P})$ & $2.348(30)$ & & & & \\
\hline $\mathrm{P}=\mathrm{O}$ & $1.512(9)$ & & & & \\
\hline $\mathrm{M}-\mathrm{O}\left(\mathrm{H}_{2} \mathrm{O}\right)$ & $2.547(16)$ & & & & \\
\hline$(\mathrm{M})-\mathrm{O}-\mathrm{S}-\mathrm{S}$ & 104.18 & & 107.13 & 107.26 & 107.56 \\
\hline $\mathrm{M}-\mathrm{O}-\mathrm{S}$ & 133.00 & & 155.45 & 155.99 & 149.52 \\
\hline $\mathrm{O}_{3} \mathrm{~S}-\mathrm{S}-\mathrm{S}$ & 104.41 & & 102.77 & 103.50 & 103.74 \\
\hline $\mathrm{O}-\mathrm{M}-\mathrm{O}$ & 76.75 & & 93.33 & 93.27 & 94.03 \\
\hline & & & & & \\
\hline
\end{tabular}

a. Values in parenthesis are standard deviations which reflect the variation in bond distances rather than uncertainties in the data collection. b. data from ref 33 , c. data from ref 14 , d. data from ref $15 \mathrm{~L}=4,4^{\prime}$-dimethyl-2,2'-bipy

The 7-membered $\mathrm{S}_{4} \mathrm{O}_{2} \mathrm{Nd}$ ring adopts a distorted twist chair conformation $[35,36]$ with the $\mathrm{NdO}_{2}$ and the two S-atoms approximately planar, and the $\mathrm{SO}_{3}$ sulfurs about $0.89 \AA$ out of plane. In this the conformation differs from that found in other tetrathionate chelates which do not adopt any of the idealised conformations of the standard cycloheptane ring [35] and instead have a planar arrangement of the $\mathrm{MO}_{2}$ and the $\mathrm{SO}_{3}$ sulfur atoms with the remaining $\mathrm{S}$-atoms between 0.67 to $0.88 \AA$ above and below this plane. The differences in the ring conformations are illustrated in Figure 5. In the transition metal complexes the tetrathionate is relatively free from steric interactions with the remaining ligand set and the conformation is presumably that which the unconstrained tetrathionate adopts. There is considerably more steric congestion in the Nd complex as illustrated in Figure 5. There are no strong interactions between the ring and the $\mathrm{Ph}_{3} \mathrm{PO}$ ligands. The closest contacts which might be considered as H-bonding interactions are between $\mathrm{O}(11)$ and the $\mathrm{H}(48), \mathrm{H}(62)$ and $\mathrm{H}(63)$-atoms of the aromatic rings with an average O....H distance of 2.714 $\AA$, but these values lie outside the sum of Van der Waals radii for O and $\mathrm{H}$ of $2.6 \AA$. 

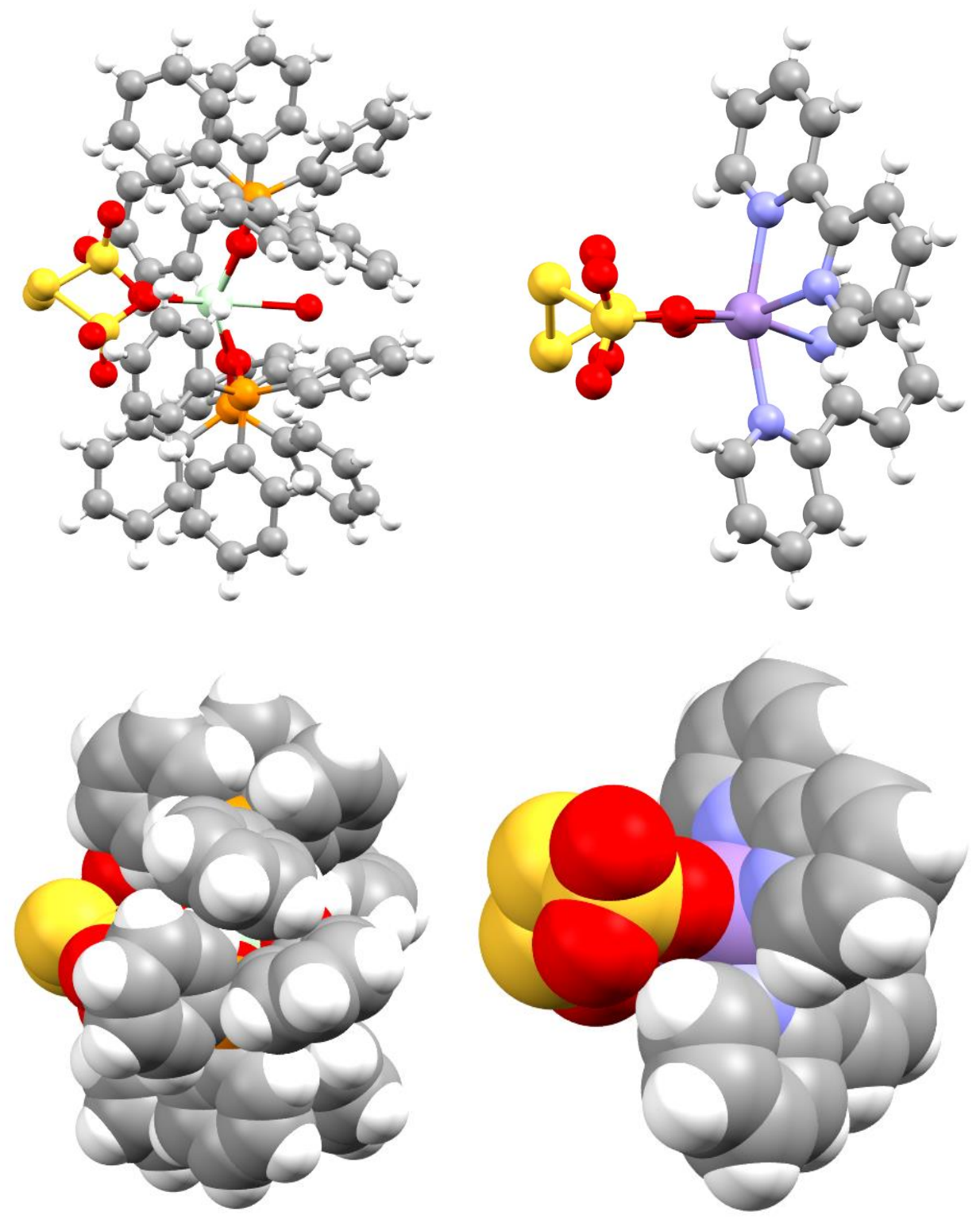

Figure 5 The ring conformation of the tetrathionate in $\left[\mathrm{Nd}\left(\mathrm{S}_{4} \mathrm{O}_{6}\right)\left(\mathrm{Ph}_{3} \mathrm{PO}\right)\left(\mathrm{H}_{2} \mathrm{O}\right]^{+}(\mathrm{left})\right.$ and $\mathrm{Mn}\left(\mathrm{S}_{4} \mathrm{O}_{6}\right)$ (bipy) $)_{2}$ (right, image generated using coordinates from ref 14) in ball and stick (upper) and space filling (lower) representations. Colour coding: sulfur - yellow, oxygen red, carbon - grey, nitrogen - lilac, manganese - purple, phosphorus - orange, neodymium pale green, hydrogen - white

The trithionate complex formed from the tetrathionate reaction mixture differs from that produced by the direct trithionate reaction in that both the cations in the unit cell are 8- rather than 9coordinate with the trithionate bonding in a $\kappa^{3}-O, O, O^{\prime}$ mode. Full details of the data collection and refinement and full listings of bond distances and angles are given in the supplementary information. The bond distances are essentially the same as found for the $\kappa^{4}$ - coordination mode and the data are given in Table 1. The 8-coordinate cation adopts a bicapped trigonal prismatic 
geometry which is illustrated for one of the molecules in the unit cell in Figure 6. The formation of a different isomer in this reaction illustrates the high sensitivity of the final product to the exact conditions during crystallisation and the relatively small energy difference between coordination modes for lanthanide complexes.

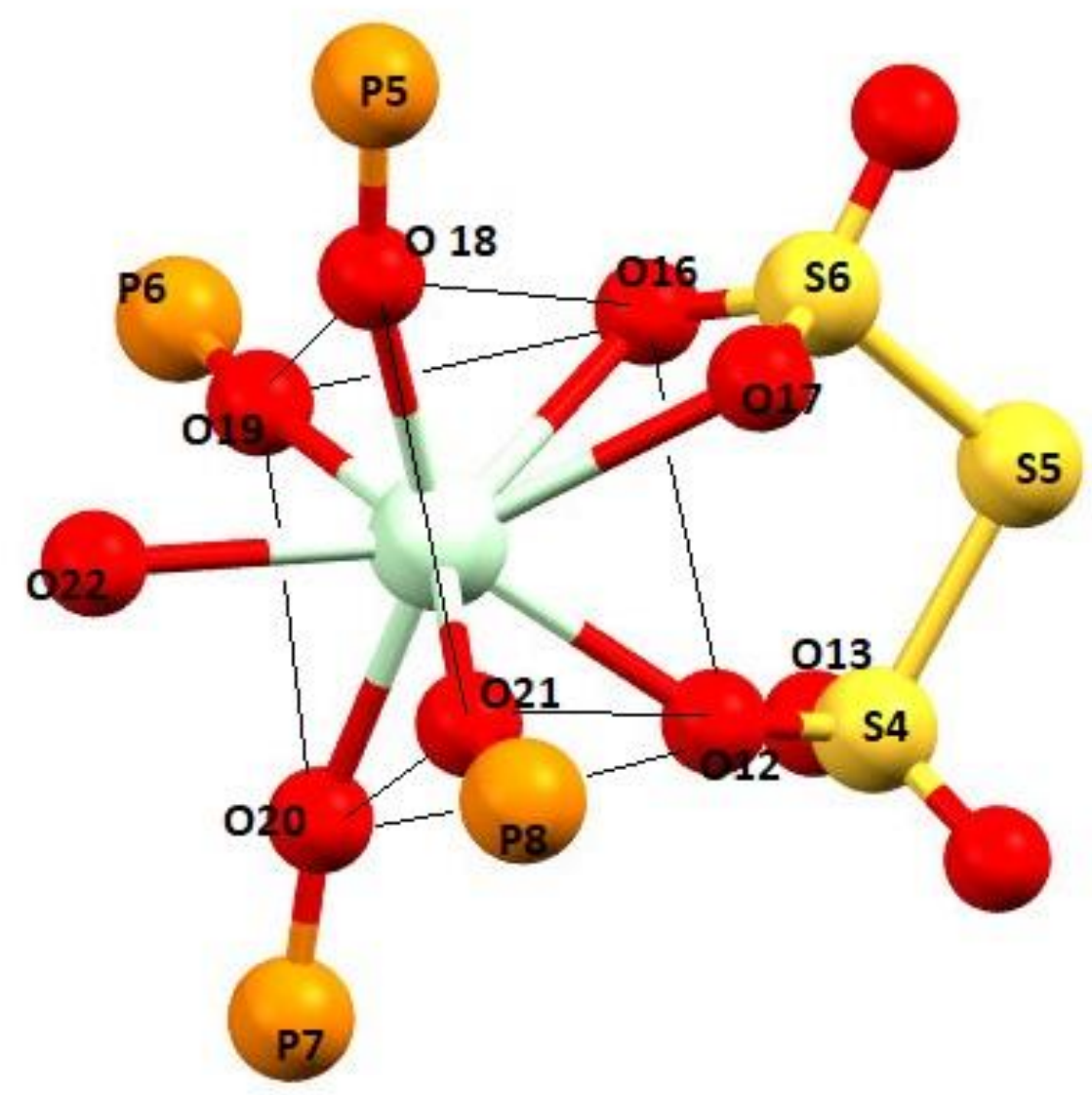

Figure 6 The bicapped trigonal prismatic core geometry of $\left[\mathrm{Nd}\left(\mathrm{S}_{3} \mathrm{O}_{6}\right)\left(\mathrm{Ph}_{3} \mathrm{PO}\right)_{4}\left(\mathrm{H}_{2} \mathrm{O}\right)\right]\left[\mathrm{ClO}{ }_{4}\right]$. Hydrogen atoms, phenyl groups and perchlorate ions omitted for clarity.

\section{Conclusion}

Lanthanide trithionates and tetrathionates have proved impossible to isolate as pure compounds. Coordination complexes of lanthanide trithionates with triphenylphosphine oxide appear to be stable for the lighter lanthanides. Whilst tetrathionate complexes with $\mathrm{Ph}_{3} \mathrm{PO}$ can be isolated in 
small amounts, the predominant reaction appears to be a disproportionation leading to the isolation of a different isomer of the trithionate complex, sulfate and elemental sulfur.

\section{Experimental}

\section{Materials and methods}

Lanthanide perchlorates were prepared from the reaction of perchloric acid with a small excess of the lanthanide oxide. Filtration followed by evaporation and drying in vacuo over $\mathrm{KOH}$ gave the hydrated perchlorates as crystalline solids.

Triphenylphosphine oxide, and potassium tetrathionate were commercial samples and used as received. Potassium trithionate was prepared by the reaction of sodium thiosulphate with hydrogen peroxide and the potassium salt obtained by metathesis with aqueous solutions of sodium trithionate and potassium acetate [37].

$\left[\mathbf{N d}\left(\mathbf{S}_{3} \mathrm{O}_{6}\right)\left(\mathbf{P h}_{3} \mathrm{PO}\right)_{4}\left(\mathbf{H}_{2} \mathrm{O}\right)\right] \mathbf{C l O}_{4} \cdot \mathbf{2 H}_{2} \mathrm{O}$ A solution of $\mathrm{Nd}\left(\mathrm{ClO}_{4}\right)_{3} 0.99 \mathrm{~g}(2.8 \mathrm{mmol})$ in $1.5 \mathrm{~mL}$ and a slurry of $\mathrm{K}_{2} \mathrm{~S}_{3} \mathrm{O}_{6}\left(0.97 \mathrm{~g}, 3.6 \mathrm{mmol}\right.$ in $\left.3 \mathrm{~mL} \mathrm{H} \mathrm{H}_{2} \mathrm{O}\right)$ were and stirred at $0^{\circ} \mathrm{C}$ for 20 minutes. Ethanol $(50 \mathrm{~mL})$ was added and stirring continued for a further 30 minutes at $0^{\circ} \mathrm{C}$. The solution was filtered into a solution of triphenylphosphine oxide $(1.99 \mathrm{~g} 7.2 \mathrm{mmol}$ in $10 \mathrm{~mL}$ ethanol). During the addition the solution became cloudy and on standing a lilac powder together with more crystalline material formed. This was filtered, washed with ethanol and dried at the pump to give 0.86 g (30\% based on $\left.\mathrm{Ph}_{3} \mathrm{PO}\right)$.

Analysis for $\left[\mathrm{Nd}\left(\mathrm{S}_{3} \mathrm{O}_{6}\right)\left(\mathrm{Ph}_{3} \mathrm{PO}\right)_{4}\right] \mathrm{ClO}_{4} \cdot 3 \mathrm{H}_{2} \mathrm{O}$ required(found) $\mathrm{C} 53.94(53.69) \quad \mathrm{H}$ 4.15(3.80) Infrared spectrum (ATR) 3364 (br, w), 3061(w), 1591(w), 1486(w), 1436(s), 1288(m), 1143(s), 1119(s), 1087(s), 1042(s), 996(s), 922(w), 752(m), 722(s), 692(s), 655(m)

$\left[\mathbf{T b}\left(\mathbf{S}_{3} \mathbf{O}_{6}\right)\left(\mathbf{P h}_{3} \mathbf{P O}\right)_{4}\left(\mathbf{H}_{2} \mathrm{O}\right)\right] \mathbf{C l O}_{4} \cdot \mathbf{2 H}_{2} \mathrm{O}$ Using the method above $\mathrm{Tb}\left(\mathrm{ClO}_{4}\right)_{3}(0.89 \mathrm{~g}, 1.6 \mathrm{mmol}$ in 2 $\mathrm{mL}$ water), $\mathrm{K}_{2} \mathrm{~S}_{3} \mathrm{O}_{6}(1.00 \mathrm{~g}, 3.7 \mathrm{mmol}$ in $3 \mathrm{~mL}$ water $)$ and $\mathrm{Ph}_{3} \mathrm{PO}(2.06 \mathrm{~g} 7.4 \mathrm{mmol}$ in $25 \mathrm{~mL})$ gave a white powder on slow evaporation of the solution; $0.67 \mathrm{~g}\left(22 \%\right.$ based on $\left.\mathrm{Ph}_{3} \mathrm{PO}\right)$. Analysis for $\left[\mathrm{Tb}\left(\mathrm{S}_{3} \mathrm{O}_{6}\right)\left(\mathrm{Ph}_{3} \mathrm{PO}\right)_{4}\left(\mathrm{H}_{2} \mathrm{O}\right)\right] \mathrm{ClO}_{4} \cdot 2 \mathrm{H}_{2} \mathrm{O}$ required(found) $\mathrm{C} 53.45(53.49) \quad \mathrm{H}$ 4.11(3.77) 
Infrared spectrum (ATR) 3350 (br, w), 3057(w), 1591(w), 1486(w), 1435(s), 1292(m), 1144(s), 1120(s), 1088(s), 1042(s), 996(s), 922(m), 750(m), 723(s), 689(s), 653(m)

$\left[\mathrm{NdS}_{4} \mathrm{O}_{6}\left(\mathrm{Ph}_{3} \mathrm{PO}\right)_{4}\left(\mathrm{H}_{2} \mathrm{O}\right)\right] \mathrm{ClO}_{4}$ The solution from $\mathrm{Nd}\left(\mathrm{ClO}_{4}\right)_{3}(0.98 \mathrm{~g}$ in $2 \mathrm{~mL}$ water $)$ and $\mathrm{K}_{2} \mathrm{~S}_{4} \mathrm{O}_{6}$ (1.66 g, $5.4 \mathrm{mmol}$ in $5.5 \mathrm{~mL}$ water) was filtered into a solution of triphenylphosphine oxide (1.84 $\mathrm{g}$ $2.8 \mathrm{mmol}$ in $18 \mathrm{~mL}$ ethanol) Crystals of $\left[\mathrm{NdS}_{4} \mathrm{O}_{6}\left(\mathrm{Ph}_{3} \mathrm{PO}\right)_{4}\left(\mathrm{H}_{2} \mathrm{O}\right)\right] \mathrm{ClO}_{4}$ suitable for X-ray diffraction $(<0.01 \mathrm{~g})$ formed at the liquid / air interface during slow evaporation of the solution. Analysis $\left[\mathrm{NdS}_{4} \mathrm{O}_{6}\left(\mathrm{Ph}_{3} \mathrm{PO}\right)_{4}\left(\mathrm{H}_{2} \mathrm{O}\right)\right] \mathrm{ClO}_{4}$ requires (found) $\mathrm{C} 54.08(53.93) \mathrm{H} 3.91(3.81)$ The bulk material, was isolated from the reduced solution by filtration as a lilac powder and mechanically separated into lilac blocks of $\left[\mathrm{Nd}\left(\mathrm{S}_{3} \mathrm{O}_{6}\right)\left(\mathrm{Ph}_{3} \mathrm{PO}\right)_{4} \mathrm{H}_{2} \mathrm{O}\right] \mathrm{ClO}_{4} \cdot 2 \mathrm{H}_{2} \mathrm{O}(0.16 \mathrm{~g})$, a microcrystalline lilac solid $\mathrm{Nd}_{2}\left(\mathrm{SO}_{4}\right)_{3} \cdot 8 \mathrm{H}_{2} \mathrm{O}(0.02 \mathrm{~g})$, and elemental sulfur.

Infrared spectra were recorded with a resolution of $\pm 1 \mathrm{~cm}^{-1}$ on a Thermo Nicolet Avatar 370 FTIR spectrometer operating in ATR mode. The samples were compressed onto the optical window and spectra recorded without further sample pre-treatment.

GC-Mass Spectrometry was carried out on Perkin Elmer Clarus 500 by manual injection of $1 \mu \mathrm{L}$ of a $\mathrm{CS}_{2}$ solution onto a Supelco SLM- 5m fused silica column (30 m x $0.32 \mathrm{~mm}$ x $0.25 \mu \mathrm{m}$ film thickness) using helium carrier gas (flow rate $1.5 \mathrm{~mL} \mathrm{~min}^{-1}$ ). The inlet temperature was $250^{\circ} \mathrm{C}$ and the oven temperature programme $180^{\circ} \mathrm{C}$ for $20 \mathrm{mins}$ and increased at $15^{\circ} \mathrm{C} \mathrm{min}^{-1}$ to $280^{\circ} \mathrm{C}$. The mass spectrometer scanned between $\mathrm{m} / \mathrm{z}$ 50-500.

Electrospray mass spectra were obtained by the EPSRC National Mass Spectrometry Service Centre at Swansea University. The spectra were recorded on a VG Quattro II triple quadrupole mass spectrometer. Samples dissolved in the appropriate solvent (water for lanthanide thoisulfates and polythionates, or $\mathrm{CH}_{2} \mathrm{Cl}_{2}$ for the triphenylphosphine oxide complexes) were loop injected into a stream of $\mathrm{MeOH}$ passing through a steel capillary held at high voltage $(+3.5 \mathrm{kV}$ for positive mode and $-3.0 \mathrm{kV}$ for negative mode). Nebulisation of the resulting spray was pneumatically assisted by a flow of nitrogen bath gas and heated source $\left(70^{\circ} \mathrm{C}\right)$. Declustering and molecular fragmentation were promoted by increasing the cone voltage from 8 to $50 \mathrm{~V}$.

\section{X-Ray crystallography}


Full details of the data collection and refinement, together with ORTEP plots showing the thermal motion at 50\% probability level are given in the supplementary information. Crystallographic data (excluding structure factors) for the structures in this paper have been deposited with the Cambridge Crystallographic Data Centre as supplementary publication numbers CCDC 1957467 for $\left[\mathrm{Er}\left(\mathrm{Ph}_{3} \mathrm{PO}\right)_{2}\left(\mathrm{H}_{2} \mathrm{O}\right)_{5}\right] \cdot 5\left(\mathrm{Ph}_{3} \mathrm{PO}\right) \cdot 3\left[\mathrm{ClO}_{4}\right], \mathrm{CCDC} 1957468$ for $\left[\mathrm{Nd}\left(\kappa^{4}-O, O, O^{\prime}, O^{\prime}-\right.\right.$ $\left.\left.\mathrm{S}_{3} \mathrm{O}_{6}\right)\left(\mathrm{Ph}_{3} \mathrm{PO}\right)_{4}\left(\mathrm{H}_{2} \mathrm{O}\right)\right] \cdot 3\left[\mathrm{ClO}_{4}\right], \mathrm{CCDC} 1957469\left[\mathrm{Nd}\left(\kappa^{2}-O, O^{\prime}-\mathrm{S}_{4} \mathrm{O}_{6}\right)\left(\mathrm{Ph}_{3} \mathrm{PO}\right)_{4}\left(\mathrm{H}_{2} \mathrm{O}\right)\right] \cdot 3\left[\mathrm{ClO}_{4}\right]$, and CCDC $1957470\left[\mathrm{Nd}\left(\kappa^{3}-O, O, O^{\prime}-\mathrm{S}_{3} \mathrm{O}_{6}\right)\left(\mathrm{Ph}_{3} \mathrm{PO}\right)_{4}\left(\mathrm{H}_{2} \mathrm{O}\right)\right] \cdot 3\left[\mathrm{ClO}_{4}\right]$ respectively. Copies of the data can be obtained, free of charge, on application to CCDC, 12 Union Road, Cambridge CB2 1EZ, UK (Fax: p44(0)-1223-336033 or e-mail: deposit@ccdc.cam.ac.uk

\section{Acknowledgements}

We are grateful to the EPSRC for the use of the National Mass Spectrometry Service at Swansea University and the National Crystallography Service at Southampton University [38].

\section{References}

1. P.V.Bernhardt, T.E.Dyahnins; Polyhedron, 23, (2004), 869

2. A.Neels, A.Alfonso, D.G.Montero, H.Stoeckli-Evans; Chimia, 57, (2003), 619

3. L.Schmitt, G.Lahat, H.Stoeckli-Evans; Acta Crystallogr. Sect C: Cryst. Struct. Comm. 66, (2010), m343

4. M.Ishii, Y.D.Daigaku Kiyo; Shiza Kagaku 15, (2001), 1

5. P.V.Bernhardt, T.W.Hambley, G.A.Lawrance; Aust. J. Chem. 43, (1990), 699

6. M.Ishii, Y.D.Daigaku Kiyo; Shiza Kagaku 15, (2001), 7

7. Y.Kim, B.W.Skelton, A.H.White; Acta Crystallogr. Sect. C: Cryst. Struct. Comm. 59, (2003), m546

8. E.B.Rusanov, V.V.Ponomarov, V.V.Komarchuk, H.Stoeckli-Evans, E.Fernadez-Ibanez, F.Stoeckli, J.Sieler, K.V.Domasevich; Angew. Chem. Int. Ed. 59, (2003), 2499

9. A.S.Degtyarenko, P.V.Solntsev, H.Krautscheid, E.B.Rusanov, A.N.Chernega,

K.V.Domasevitch; New J. Chem. 32, (2008), 1910

10. S.Turba, O.Walter, S.Schindler, L.P.Nielsen, A.Hazell, C.J.McKenzie, F.Lloret, J.Cano, M.Julve; Inorg. Chem. 47, (2008), 1612

11. E.Lork, R.Mews, A.V.Zibarev; Mendeleev Comm 19, (2009) 147

12. E.Freire, S.Baggio, R.Baggio, M.T.Garland; ActCrystallogr.,Sect.C:Cryst.Struct.Commun. 54, (1998), 464 
13. E.R.T.Tiekinka; Acta Crystallogr.Sect E: Structure Reports Online 65, (2009), i89

14. E.Freire, S.Baggio, R.Baggio; A.Mombru; Acta Crystallogr.,Sect.C:Cryst.Struct.Commun. 57, (2001), 14

15. M.A.Harvey, S.Suarez, F.Doctorovich, R.Baggio; Crystallogr., Sect.C: Cryst.Struct.Commun. 69, (2013), 745

16. J.Fawcett, A.W.G.Platt and D.R.Russell; Inorg. Chim. Acta 274, (1998) 177

17.R.S.Hughes, J.Fawcett, A.W.G.Platt and D.R.Russell; Inorg. Chim. Acta 295, (1999), 146

18. K.Nakamoto; Infrared and Raman Spectra of Inorganic and Coordination Compounds $6^{\text {th }}$ Ed John Wiley and Sons, New York (2009)

19. H.Zhang, M.L.Jeffrey; Inorg. Chem. 49, (2010), 10273

20. R.Colton, A.D’Agostino, J.C.Traeger; Mass Spectrom. Rev. 14, (1995), 79

21. M.C.Etter, P.W.Baures; J.Amer.Chem.Soc. 110, (1988), 639

22. A.W.G.Platt; Coord. Chem. Rev. 340, (2017), 62

23. P. C. Christidis, P. J. Rentzeperis; Z. Kristallogr., 173, (1985) 59

24. M.Llunell, D.Casanova, J.Cirera, P.Alemany, S.Alvarez; SHAPE - Program for the Stereochemical Analysis of Molecular Fragments by Means of Continuous Shape Measures and Associated Tools, Version 2.1, University of Barcelona, Spain, 2013Shape

25. S.Alvarez, P.Alemany, D.Casanova, J.Cirera, M.Llunell, D.Avnir; Coord. Chem. Rev. 249, (2005), 1693

26. A. Ruiz-Martinez, D.Casanova, S. Alvarez; Chem. Eur. J. 14, (2008), 1291

27. S.J. Coles M. B. Pitak and A. W. G. Platt; Eur. J. Inorg. Chem (2017) 1464

28. A.M.J.Lees, A.W.G.Platt; Polyhedron 67, (2014), 368

29. Y.-C.Chen, J-L.Lui, L.Ungur, Q.-W.Li, L.-F.Wang, Z.-P.Ni, L.F.Chibotaru, X.-M.Chen, M.L.Tong; J. Amer. Chem. Soc. 138, (2016), 2829

30. A.Bowden, A.M.J.Lees, A.W.G.Platt; Polyhedron 91, (2015), 110

31. Z-Y.Li, C.Zhang, F-Q.Zhang, X-F.Zhang, S-Z.Li, G-X.Cao, B.Zhai; J. Mol. Struct. 1108, (2016), 516

32. Z.Deng, F.Bai, Y.Xing, N.Xing, L.Xu; Open J. Inorg. Chem. 3, (2013), 7 
33. D.Casanova, P.Alemany, J.M.Bofill, S.Alvarez; Chem. Eur. J. 9, (2003),1281

34. P. C. Christidis, P. J. Rentzeperis, A. Kirfel, G. Will; Z. Kristallogr., 188, (1989),

35. A. Entrena, J.M.Campos, M.A.Gallo, A.Espinosa; ARKIVOC vi, (2005), 88

36. I.K.Boessenkool, J.C.A. Boeyens; J. Cryst. Mol. Struct. 10, (1980), 11

37. D.P.Kelly A.P.Wood; Methods in Enzymology 243, (1994), 475

38. S.J.Coles, P.A.Gale; Chem. Sci. 3, (2012), 68 


\section{Supplementary Information}

Table S1 Electrospray mass spectra ${ }^{\mathrm{a}}$ of lanthanide tri- and tetrathionate species; positive ion mode

\begin{tabular}{|c|c|c|c|c|c|c|c|c|c|}
\hline & & $\mathrm{n}_{2}\left(\mathrm{~S}_{3} \mathrm{O}_{6}\right)$ & & & & & & $2\left(\mathrm{~S}_{4} \mathrm{O}_{6}\right)_{3}$ & \\
\hline$\left[\mathrm{Ln}\left(\mathrm{S}_{3} \mathrm{O}_{6}\right)(\mathrm{Me}\right.$ & $\mathrm{m}\left(\mathrm{H}_{2} \mathrm{O}\right)_{\mathrm{n}}$ & & & & & & $\left(\mathrm{S}_{4} \mathrm{O}_{6}\right)(\mathrm{I}$ & $\mathrm{eOH})_{\mathrm{m}}($ & $\left.\left.{ }_{2} \mathrm{O}\right)_{\mathrm{n}}\right]^{+}$ \\
\hline $\mathrm{m}=0$ & & & & & & & & & \\
\hline $\mathrm{n}$ & 0 & 1 & 2 & 3 & 4 & $\mathrm{n}$ & 1 & 2 & 3 \\
\hline $\operatorname{Pr}$ & & & & & & & & & \\
\hline $\mathrm{Nd}$ & & & $\begin{array}{l}369.8^{b} \\
(369.8)\end{array}$ & $\begin{array}{l}387.9 \\
(387.9)\end{array}$ & $\begin{array}{l}405.8 \\
(405.8)\end{array}$ & & $\begin{array}{l}383.7 \\
(383.8)\end{array}$ & $\begin{array}{l}401.7 \\
(401.8)\end{array}$ & $\begin{array}{l}419.8 \\
(419.8)\end{array}$ \\
\hline $\mathrm{m}=1$ & & & & & & & & & \\
\hline $\operatorname{Pr}$ & & $\begin{array}{l}382.8 \\
(382.8)\end{array}$ & $\begin{array}{l}400.8 \\
(400.8)\end{array}$ & $\begin{array}{l}418.8 \\
(419.2)\end{array}$ & $\begin{array}{l}436.9 \\
(436.9)\end{array}$ & & & & \\
\hline $\mathrm{Nd}$ & & $\begin{array}{l}384.0 \\
(383.8)\end{array}$ & $\begin{array}{l}401.9 \\
(401.8)\end{array}$ & $\begin{array}{l}419.9 \\
(419.9)\end{array}$ & & & & & \\
\hline $\mathrm{m}=2$ & & & & & & & & & \\
\hline $\operatorname{Pr}$ & $\begin{array}{l}396.8 \\
(396.8)\end{array}$ & $\begin{array}{l}414.8 \\
(414.9)\end{array}$ & $\begin{array}{l}432.8 \\
(432.9)\end{array}$ & & & & & & \\
\hline $\mathrm{Nd}$ & & & & & & & & & \\
\hline$\left[\mathrm{LnO}\left(\mathrm{H}_{2} \mathrm{O}\right)_{\mathrm{n}}\right]^{+}$ & & & & & & & & & \\
\hline $\operatorname{Pr}$ & $\begin{array}{l}156.8 \\
(156.9)\end{array}$ & $\begin{array}{l}174.8 \\
(174.9)\end{array}$ & & & & & & & \\
\hline $\mathrm{Nd}$ & $\begin{array}{l}157.9 \\
(157.9)\end{array}$ & $\begin{array}{l}175.7 \\
(175.9)\end{array}$ & $\begin{array}{l}193.7 \\
(193.9)\end{array}$ & & & & & & \\
\hline
\end{tabular}




\begin{tabular}{|l|l|l|l|l|l|l|l|l|l|}
\hline$\left[\mathrm{LnO}(\mathrm{MeOH})_{\mathrm{n}}\right]^{+}$ & & & & & & & & & \\
\hline Pr & & 188.8 & 220.9 & & & & & & \\
& & $(188.9)$ & $(221.0)$ & & & & & & \\
\hline Nd & & 189.7 & & & & & & & \\
& & $(189.9)$ & & & & & & & \\
\hline
\end{tabular}

a. The most intense peak in the isotope profile is quoted.

b. $\mathrm{m} / \mathrm{z}$ observed (calculated) 
Table S2 Electrospray mass spectra ${ }^{a}$ of of lanthanide tri- and tetrathionate species; negative ion mode

\begin{tabular}{|c|c|c|c|c|c|c|}
\hline$\left[\mathrm{Ln}\left(\mathrm{S}_{3} \mathrm{O}_{6}\right)_{2}\left(\mathrm{H}_{2} \mathrm{O}\right)_{\mathrm{n}}\right]^{-}$ & $\mathrm{n}$ & 0 & 1 & & & \\
\hline $\operatorname{Pr}$ & & $\begin{array}{l}524.0^{b} \\
(524.7)\end{array}$ & & & & \\
\hline $\mathrm{Nd}$ & & $\begin{array}{l}527.8 \\
(527.7)\end{array}$ & $\begin{array}{l}545.7 \\
(545.7)\end{array}$ & & & \\
\hline$\left[\mathrm{Ln}\left(\mathrm{S}_{3} \mathrm{O}_{6}\right)_{2}(\mathrm{MeOH})_{\mathrm{n}}\right]^{-}$ & $\mathrm{n}$ & 1 & 2 & 3 & 4 & 5 \\
\hline $\operatorname{Pr}$ & & $\begin{array}{l}556.8 \\
(556.7)\end{array}$ & $\begin{array}{l}588.7 \\
(588.7)\end{array}$ & $\begin{array}{l}620.6 \\
(620.8)\end{array}$ & $\begin{array}{l}652.6 \\
(652.9)\end{array}$ & $\begin{array}{l}684.6 \\
(684.8)\end{array}$ \\
\hline $\mathrm{Nd}$ & & $\begin{array}{l}557.7 \\
(557.7)\end{array}$ & $\begin{array}{l}589.6 \\
(589.7)\end{array}$ & & & \\
\hline$\left[\mathrm{S}_{3} \mathrm{O}_{6}(\mathrm{MeOH})_{\mathrm{m}}\right]^{2-}$ & & $\begin{array}{l}111.9 \\
(112.0)\end{array}$ & $\begin{array}{l}127.9 \\
(128.0)\end{array}$ & $\begin{array}{l}143.8 \\
(143.7)\end{array}$ & $\begin{array}{l}159.8 \\
(160.0)\end{array}$ & $\begin{array}{l}175.8 \\
(176.0)\end{array}$ \\
\hline
\end{tabular}

a. The most intense peak in the isotope profile quoted for $\mathrm{Nd}$ containing ions

b. $\mathrm{m} / \mathrm{z}$ observed (calculated) 
Figure S1 Comparative infrared spectra of $\left[\kappa^{3}-O, O, O^{\prime}-\mathrm{Nd}\left(\mathrm{S}_{3} \mathrm{O}_{6}\right)\left(\mathrm{Ph}_{3} \mathrm{PO}\right)_{4}\left(\mathrm{H}_{2} \mathrm{O}\right)\right] \mathrm{ClO}_{4}($ red $)$ and $\left[\kappa^{A}-O, O, O^{\prime}, O^{\prime}-\mathrm{Nd}\left(\mathrm{S}_{3} \mathrm{O}_{6}\right)\left(\mathrm{H}_{2} \mathrm{O}\right)\left(\mathrm{Ph}_{3} \mathrm{PO}\right)_{4}\right] \mathrm{ClO}_{4}$ (blue)

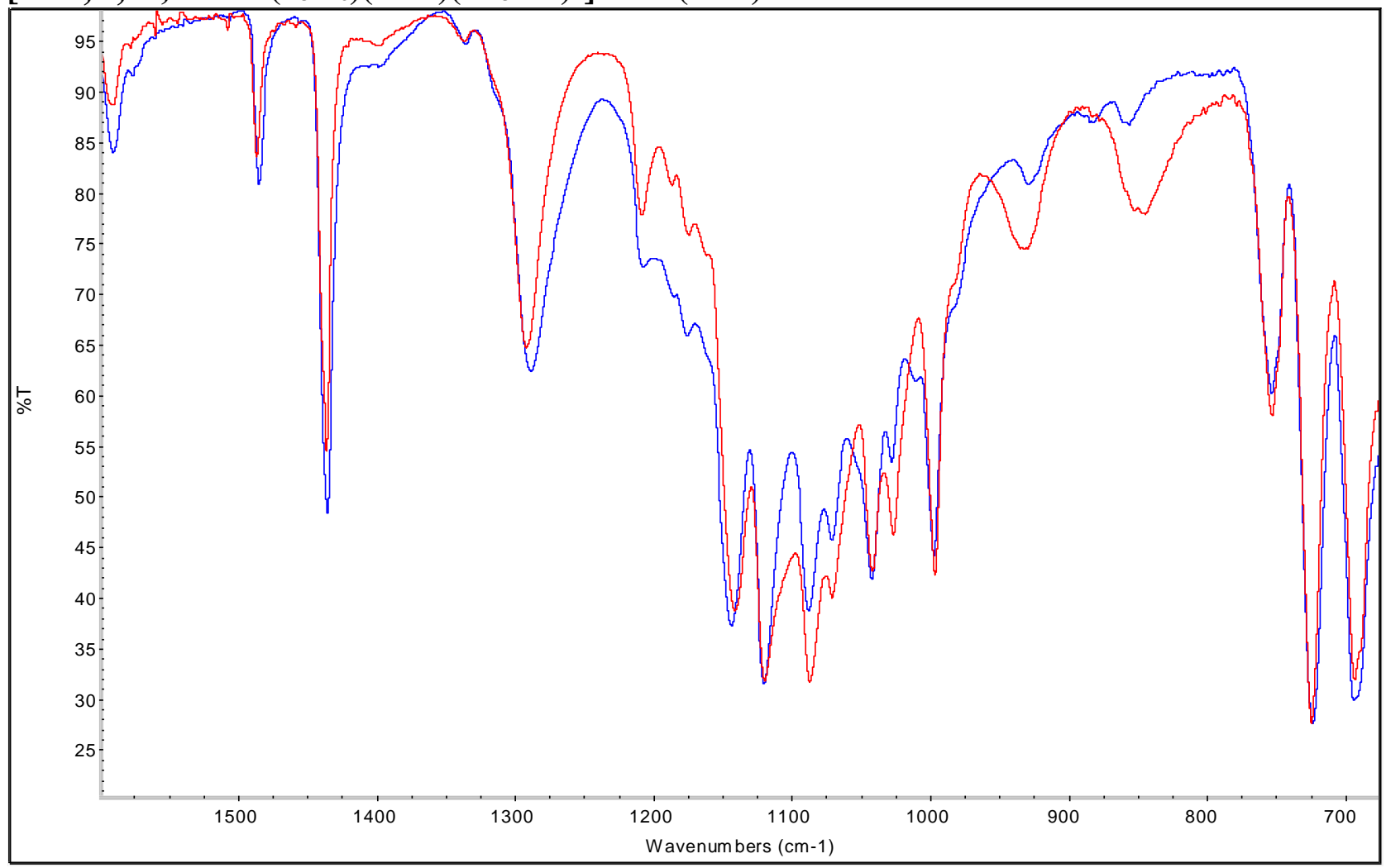


Table S3 Electrospray mass spectra of $\left[\mathrm{Ln}\left(\mathrm{S}_{4} \mathrm{O}_{6}\right)\left(\mathrm{Ph}_{3} \mathrm{PO}\right)_{4} \mathrm{H}_{2} \mathrm{O}\right]\left[\mathrm{ClO}_{4}\right]$

\begin{tabular}{|c|c|c|c|c|c|c|}
\hline & \multicolumn{2}{|c|}{$\left[\mathrm{Ln}\left(\mathrm{S}_{4} \mathrm{O}_{6}\right)\left(\mathrm{Ph}_{3} \mathrm{PO}\right)_{\mathrm{n}}\right]^{+}$} & \multicolumn{2}{|c|}{$\left[\mathrm{Ln}\left(\mathrm{Ph}_{3} \mathrm{PO}\right)_{\mathrm{n}} \mathrm{ClO}_{4}\right]^{2+}$} & \multicolumn{2}{|c|}{$\left[\mathrm{Ln}\left(\mathrm{Ph}_{3} \mathrm{PO}\right)_{\mathrm{n}} \mathrm{ClO}_{4}\right]^{2+}$} \\
\hline $\mathrm{Ln}$ & $\operatorname{Pr}$ & $\mathrm{Nd}$ & $\operatorname{Pr}$ & $\mathrm{Nd}$ & $\operatorname{Pr}$ & $\mathrm{Nd}$ \\
\hline 1 & & & & & & \\
\hline 2 & & & & & $232.4(232.3)$ & $232.4(233.3)$ \\
\hline 3 & $\begin{array}{l}1199.3 \\
(1199.0)\end{array}$ & $1202.4(1202.0)$ & $537.3(537.0)$ & $538.8(538.5)$ & $325.2(325.0)$ & $326.0(326.0)$ \\
\hline 4 & & & $676.3(676.0)$ & $677.7(677.5)$ & $418.0(417.7)$ & $418.9(418.7)$ \\
\hline 5 & & & & & $510.8(510.3)$ & $511.5(511.3)$ \\
\hline 6 & & & & & $603.5(603.3)$ & $604.4(604.0)$ \\
\hline
\end{tabular}




\section{Crystal structures}




\section{Crystal Data and Experimental $\left[\mathrm{Nd}\left(\mathrm{K}^{4}-\mathrm{O}, O, O^{\prime}, O^{\prime}-\right.\right.$ $\left.\left.\mathrm{S}_{3} \mathrm{O}_{6}\right)\left(\mathrm{Ph}_{3} \mathrm{PO}\right)_{4}\left(\mathrm{H}_{2} \mathrm{O}\right)\right] \cdot 3\left[\mathrm{ClO}_{4}\right]$}

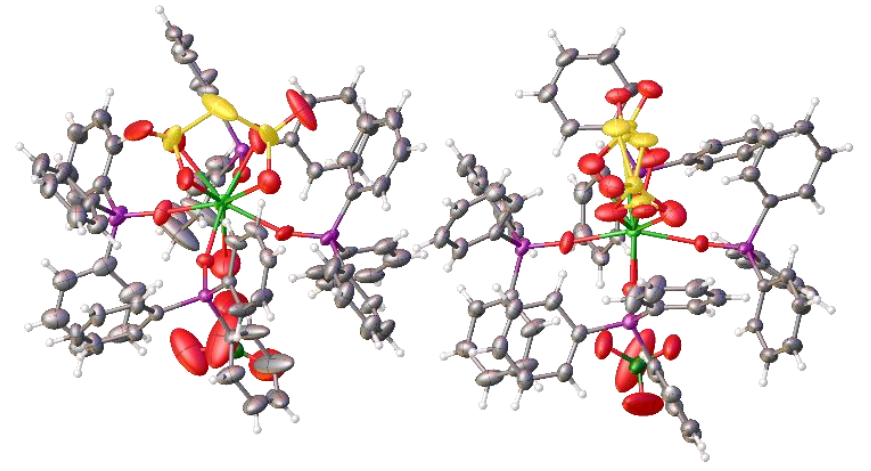

Figure 1: Thermal ellipsoids drawn at the $50 \%$ probability level.

Experimental. A suitable crystal of ssf1275 $\left(0.150 \times 0.100 \times 0.080 \mathrm{~mm}^{3}\right)$ was selected and mounted on a suitable support on an Daresbury SRS station 9.8 diffractometer. The crystal was kept at a steady $T=$ 120(2) K during data collection. The structure was solved with the ShelXT (Sheldrick, 2015) structure solution program using the Intrinsic Phasing solution method and by using Olex2 (Dolomanov et al., 2009) as the graphical interface. The model was refined with version 2014/7 of ShelXL (Sheldrick, 2015) using Least Squares minimisation.

Crystal Data. $\mathrm{C}_{72} \mathrm{H}_{60} \mathrm{ClNdO}_{15} \mathrm{P}_{4} \mathrm{~S}_{3}, \quad M_{r}=1564.95$, monoclinic, $C 2$ (No. 5), a = 19.8173(9) $\AA$, b = 17.2962(8) $\AA$, $\mathrm{c}=19.6261(9) \AA, \quad \beta=90.695(2)^{\circ}, \quad \alpha=\gamma=90^{\circ}, \quad V=$ 6726.6(5) $\AA^{3}, T=120(2) \mathrm{K}, Z=4, Z^{\prime}=1, \mu=1.005 \mathrm{~mm}^{-1}$, 33755 reflections measured, 18225 unique $\left(R_{\text {int }}=0.0530\right)$ which were used in all calculations. The final $w R_{2}$ was 0.1630 (all data) and $R_{1}$ was 0.0614 (I > 2(I)).

\begin{tabular}{|c|c|}
\hline Formula & $\mathrm{C}_{72} \mathrm{H}_{60} \mathrm{ClNdO}_{15} \mathrm{P}_{4} \mathrm{~S}_{3}$ \\
\hline$D_{\text {calc. }} / \mathrm{g} \mathrm{cm}^{-3}$ & 1.545 \\
\hline$\mu / \mathrm{mm}^{-1}$ & 1.005 \\
\hline Formula Weight & 1564.95 \\
\hline Colour & $?$ \\
\hline Shape & ? \\
\hline Size $/ \mathrm{mm}^{3}$ & $0.150 \times 0.100 \times 0.080$ \\
\hline$T / K^{\prime}$ & $120(2)$ \\
\hline Crystal System & monoclinic \\
\hline Flack Parameter & $0.479(13)$ \\
\hline Hooft Parameter & $0.477(6)$ \\
\hline Space Group & $C 2$ \\
\hline$a / \AA$ & $19.8173(9)$ \\
\hline$b / \AA$ & $17.2962(8)$ \\
\hline$c / \AA$ & $19.6261(9)$ \\
\hline$\alpha /^{\circ}$ & 90 \\
\hline$\beta /^{\circ}$ & $90.695(2)$ \\
\hline$\gamma /^{\circ}$ & 90 \\
\hline $\mathrm{V} / \AA^{3}$ & $6726.6(5)$ \\
\hline$Z^{\prime}$ & 4 \\
\hline$Z^{\prime}$ & 1 \\
\hline Wavelength/Å & 0.6926 \\
\hline Radiation type & Synchrotron \\
\hline$\Theta_{\min } /^{\circ}$ & 2.255 \\
\hline$\Theta_{\max } /^{\circ}$ & 29.672 \\
\hline Measured Refl. & 33755 \\
\hline Independent Refl. & 18225 \\
\hline $\begin{array}{l}\text { Reflections with I > } \\
2(\mathrm{I})\end{array}$ & 16848 \\
\hline Rint & 0.0530 \\
\hline Parameters & 892 \\
\hline Restraints & 808 \\
\hline Largest Peak & 1.895 \\
\hline Deepest Hole & -1.471 \\
\hline GooF & 1.042 \\
\hline$w R_{2}$ (all data) & 0.1630 \\
\hline$w R_{2}$ & 0.1590 \\
\hline$R_{1}$ (all data) & 0.0654 \\
\hline$R_{1}$ & 0.0614 \\
\hline
\end{tabular}


Structure Quality Indicators

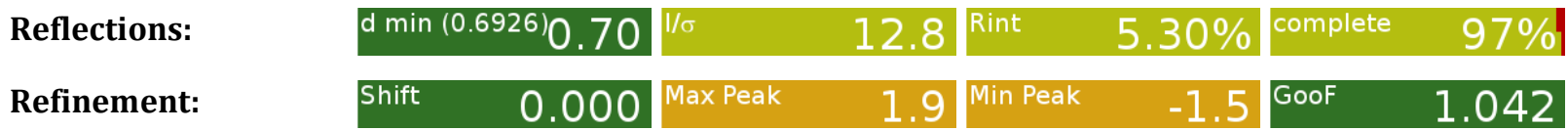

A suitable crystal with dimensions $0.150 \times 0.100 \times 0.080 \mathrm{~mm}^{3}$ was mounted on a suitable support. Data were collected using the Daresbury SRS station 9.8 diffractometer, equipped with a $\mathrm{Si}(111)$ monochromator and Bruker SMART APEX2 CCD detector (Bruker, 2004), and operating at T = 120(2) K.

Data were measured using synchrotron radiation at a wavelength of $0.6911 \AA$ A The maximum resolution that was achieved was $\Theta=29.672^{\circ}(0.70 \AA)$.

The diffraction pattern was indexed using SAINT (Bruker, 2004).

Data reduction, scaling and absorption corrections were performed using Bruker SAINT. The final completeness is $98.30 \%$ out to $29.672^{\circ}$ in $\Theta$. An empirical absorption correction was performed using SADABS (Bruker, 2004). The absorption coefficient $\mu$ of this material is $1.005 \mathrm{~mm}^{-1}$ at this wavelength $(\lambda=$ $0.6926 \AA ̊)$.

The structure was solved and the space group C2 (\# 5) determined by the ShelXT (Sheldrick, 2015) structure solution program using Intrinsic Phasing and refined by Least Squares using version 2014/7 of ShelXL (Sheldrick, 2015). All non-hydrogen atoms were refined anisotropically. Hydrogen atom positions were calculated geometrically and refined using the riding model. The Nd2 S3 bridge is disordered, and treated as disordered. The Nd1 S3 bride also seems disordered, but has not been treated as such.

There are 2 half molecules in the asymmetric unit, which is represented by the reported sum formula. In other words: $\mathrm{Z}$ is 4 and $\mathrm{Z}^{\prime}$ is 1 .

The Flack parameter was refined to $0.479(13)$. Determination of absolute structure using Bayesian statistics on Bijvoet differences using the Olex2 results in 0.477(6). Note: The Flack parameter is used to determine chirality of the crystal studied, the value should be near 0 , a value of 1 means that the stereochemistry is wrong and the model should be inverted. A value of 0.5 means that the crystal consists of a racemic mixture of the two enantiomers. There is inverse twinning, approximately half-half.

\section{Reflection Statistics}

$\begin{array}{ll}\begin{array}{l}\text { Total reflections (after } \\ \text { filtering) }\end{array} & 33755 \\ \text { Completeness } & 0.886 \\ \mathrm{hkl}_{\max } \text { collected } & (27,24,27) \\ \mathrm{hkl}_{\max } \text { used } & (27,24,27) \\ \text { Lim } \mathrm{d}_{\max } \text { collected } & 100.0 \\ \mathrm{~d}_{\max } \text { used } & 8.8 \\ \text { Friedel pairs } & 12302 \\ \text { Inconsistent equivalents } & 0 \\ \mathrm{R}_{\text {sigma }} & 0.0782 \\ \text { Omitted reflections } & 0 \\ \text { Multiplicity } & (28151,2661,94) \\ \text { Removed systematic absences } & 0\end{array}$

$\begin{array}{ll}\text { Unique reflections } & 18225 \\ \text { Mean I/ } \sigma & 9.57 \\ \mathrm{hkl}_{\text {min }} \text { collected } & (-27,-24,-27) \\ \mathrm{hkl}_{\text {min }} \text { used } & (-27,-24,0) \\ \text { Lim } \mathrm{d}_{\text {min }} \text { collected } & 0.35 \\ \mathrm{~d}_{\min } \text { used } & 0.7 \\ \text { Friedel pairs merged } & 0 \\ \mathrm{R}_{\text {int }} & 0.053 \\ \text { Intensity transformed } & 0 \\ \text { Omitted by user (OMIT hkl) } & 0 \\ \text { Maximum multiplicity } & 5 \\ \text { Filtered off (Shel/OMIT) } & 0\end{array}$


Table 1: Bond Lengths in Å.

\begin{tabular}{|c|c|c|}
\hline Atom & Atom & Length/Å \\
\hline$\overline{\mathrm{Nd} 1}$ & $01^{1}$ & $2.349(5)$ \\
\hline $\mathrm{Nd} 1$ & 01 & $2.349(5)$ \\
\hline $\mathrm{Nd} 1$ & 02 & $2.311(5)$ \\
\hline $\mathrm{Nd} 1$ & $02^{1}$ & $2.311(5)$ \\
\hline $\mathrm{Nd} 1$ & 05 & $2.743(15)$ \\
\hline Nd1 & $07^{1}$ & $2.686(10)$ \\
\hline $\mathrm{Nd} 1$ & 07 & $2.686(10)$ \\
\hline $\mathrm{Nd} 1$ & 091 & $2.499(8)$ \\
\hline $\mathrm{Nd} 1$ & 09 & $2.499(8)$ \\
\hline $\mathrm{Nd} 2$ & $\mathrm{~S}^{2}$ & $3.237(4)$ \\
\hline $\mathrm{Nd} 2$ & S3 & $3.237(4)$ \\
\hline $\mathrm{Nd} 2$ & 03 & $2.330(5)$ \\
\hline $\mathrm{Nd} 2$ & $03^{2}$ & $2.330(5)$ \\
\hline $\mathrm{Nd} 2$ & $04^{2}$ & $2.362(5)$ \\
\hline $\mathrm{Nd} 2$ & 04 & $2.362(5)$ \\
\hline $\mathrm{Nd} 2$ & 06 & $2.550(7)$ \\
\hline $\mathrm{Nd} 2$ & 010 & $2.654(9)$ \\
\hline $\mathrm{Nd} 2$ & $010^{2}$ & $2.654(9)$ \\
\hline $\mathrm{Nd} 2$ & $011^{2}$ & $2.550(8)$ \\
\hline $\mathrm{Nd} 2$ & 011 & $2.550(8)$ \\
\hline $\mathrm{Cl} 1$ & $013^{1}$ & $1.363(16)$ \\
\hline $\mathrm{Cl} 1$ & 013 & $1.363(16)$ \\
\hline $\mathrm{Cl} 1$ & 014 & $1.471(17)$ \\
\hline $\mathrm{Cl} 1$ & $014^{1}$ & $1.471(17)$ \\
\hline $\mathrm{Cl} 2$ & $015^{2}$ & $1.423(7)$ \\
\hline $\mathrm{Cl} 2$ & 015 & $1.423(7)$ \\
\hline $\mathrm{Cl} 2$ & $016^{2}$ & $1.364(13)$ \\
\hline $\mathrm{Cl} 2$ & 016 & $1.364(13)$ \\
\hline S5 & $\mathrm{S} 4^{2}$ & $2.118(7)$ \\
\hline S5 & S4 & $1.558(6)$ \\
\hline S5 & $010^{2}$ & $1.955(11)$ \\
\hline S5 & $011^{2}$ & $1.529(8)$ \\
\hline S5 & $012 A^{2}$ & $1.473(13)$ \\
\hline S2 & $\mathrm{S} 1^{1}$ & $2.079(4)$ \\
\hline S2 & $\mathrm{S} 1$ & $2.079(4)$ \\
\hline S4 & $\mathrm{S} 5^{2}$ & $2.118(7)$ \\
\hline S4 & $\mathrm{S} 4^{2}$ & $1.138(11)$ \\
\hline S1 & 07 & $1.578(10)$ \\
\hline S1 & 08 & $1.403(8)$ \\
\hline S1 & 09 & $1.425(8)$ \\
\hline S3 & 010 & $1.439(11)$ \\
\hline S3 & 011 & $1.277(9)$ \\
\hline S3 & 012 & $1.425(12)$ \\
\hline P1 & 01 & $1.507(5)$ \\
\hline P1 & $\mathrm{C} 1$ & $1.805(6)$ \\
\hline P1 & $\mathrm{C} 7$ & $1.785(7)$ \\
\hline P1 & C13 & $1.795(6)$ \\
\hline P2 & 02 & $1.510(6)$ \\
\hline P2 & C19 & $1.772(9)$ \\
\hline P2 & $\mathrm{C} 25$ & $1.801(8)$ \\
\hline P2 & C31 & $1.775(8)$ \\
\hline P3 & 03 & $1.503(5)$ \\
\hline P3 & $\mathrm{C} 37$ & $1.794(6)$ \\
\hline P3 & C43 & $1.806(7)$ \\
\hline P3 & C49 & $1.784(8)$ \\
\hline P4 & 04 & $1.504(5)$ \\
\hline P4 & C55 & $1.793(7)$ \\
\hline P4 & C61 & $1.793(7)$ \\
\hline P4 & $\mathrm{C} 67$ & $1.804(7)$ \\
\hline 010 & $\mathrm{~S} 5^{2}$ & $1.955(11)$ \\
\hline
\end{tabular}

\begin{tabular}{|c|c|c|}
\hline Atom & Atom & Length/Å \\
\hline$\overline{011}$ & $\mathrm{~S}^{2}$ & $1.529(8)$ \\
\hline 012A & $\mathrm{S} 5^{2}$ & $1.473(13)$ \\
\hline 013 & $013^{1}$ & $1.76(4)$ \\
\hline $\mathrm{C} 1$ & $\mathrm{C} 2$ & $1.370(9)$ \\
\hline C1 & C6 & $1.389(10)$ \\
\hline $\mathrm{C} 2$ & $\mathrm{C} 3$ & $1.397(10)$ \\
\hline C3 & $\mathrm{C} 4$ & $1.350(12)$ \\
\hline $\mathrm{C} 4$ & $\mathrm{C} 5$ & $1.361(12)$ \\
\hline $\mathrm{C} 5$ & C6 & $1.388(11)$ \\
\hline $\mathrm{C} 7$ & $\mathrm{C} 8$ & $1.423(12)$ \\
\hline $\mathrm{C} 7$ & $\mathrm{C} 12$ & $1.325(13)$ \\
\hline $\mathrm{C} 8$ & $\mathrm{C} 9$ & $1.389(13)$ \\
\hline $\mathrm{C} 9$ & C10 & $1.388(19)$ \\
\hline C10 & C11 & $1.32(2)$ \\
\hline C11 & $\mathrm{C} 12$ & $1.424(16)$ \\
\hline C13 & C14 & $1.398(9)$ \\
\hline C13 & C18 & $1.391(9)$ \\
\hline C14 & C15 & $1.400(9)$ \\
\hline C15 & C16 & $1.389(12)$ \\
\hline C16 & $\mathrm{C} 17$ & $1.376(11)$ \\
\hline C17 & C18 & $1.405(9)$ \\
\hline C19 & $\mathrm{C} 20$ & $1.389(12)$ \\
\hline C19 & $\mathrm{C} 24$ & $1.435(15)$ \\
\hline C20 & $\mathrm{C} 21$ & $1.404(14)$ \\
\hline C21 & $\mathrm{C} 22$ & $1.382(17)$ \\
\hline $\mathrm{C} 22$ & $\mathrm{C} 23$ & $1.413(16)$ \\
\hline C23 & $\mathrm{C} 24$ & $1.346(15)$ \\
\hline C25 & $\mathrm{C} 26$ & $1.399(10)$ \\
\hline $\mathrm{C} 25$ & C30 & $1.397(12)$ \\
\hline C26 & $\mathrm{C} 27$ & $1.396(11)$ \\
\hline $\mathrm{C} 27$ & $\mathrm{C} 28$ & $1.382(13)$ \\
\hline C28 & $\mathrm{C} 29$ & $1.419(14)$ \\
\hline C29 & C30 & $1.382(12)$ \\
\hline C31 & C32 & $1.377(10)$ \\
\hline C31 & C36 & $1.394(11)$ \\
\hline C32 & C33 & $1.396(14)$ \\
\hline C33 & C34 & $1.349(17)$ \\
\hline C34 & C35 & $1.392(14)$ \\
\hline C35 & C36 & $1.381(13)$ \\
\hline C37 & C38 & $1.395(9)$ \\
\hline C37 & $\mathrm{C} 42$ & $1.402(9)$ \\
\hline C38 & C39 & $1.395(9)$ \\
\hline C39 & $\mathrm{C} 40$ & $1.386(12)$ \\
\hline $\mathrm{C} 40$ & $\mathrm{C} 41$ & $1.418(13)$ \\
\hline C41 & $\mathrm{C} 42$ & $1.367(10)$ \\
\hline C43 & $\mathrm{C} 44$ & $1.399(11)$ \\
\hline C43 & $\mathrm{C} 48$ & $1.388(10)$ \\
\hline C44 & $\mathrm{C} 45$ & $1.381(11)$ \\
\hline $\mathrm{C} 45$ & $\mathrm{C} 46$ & $1.378(12)$ \\
\hline C46 & $\mathrm{C} 47$ & $1.381(12)$ \\
\hline C47 & $\mathrm{C} 48$ & $1.392(10)$ \\
\hline C49 & C50 & $1.396(11)$ \\
\hline C49 & C54 & $1.398(10)$ \\
\hline C50 & C51 & $1.395(11)$ \\
\hline C51 & C52 & $1.392(12)$ \\
\hline C52 & C53 & $1.373(15)$ \\
\hline C53 & C54 & $1.377(13)$ \\
\hline C55 & C56 & $1.404(11)$ \\
\hline C55 & $\mathrm{C} 60$ & $1.380(10)$ \\
\hline C56 & C57 & $1.376(11)$ \\
\hline
\end{tabular}




\begin{tabular}{lll}
\hline Atom & Atom & Length/Å \\
\hline C57 & C58 & $1.383(14)$ \\
C58 & C59 & $1.373(13)$ \\
C59 & C60 & $1.384(10)$ \\
C61 & C62 & $1.419(11)$ \\
C61 & C66 & $1.380(10)$ \\
C62 & C63 & $1.384(12)$ \\
C63 & C64 & $1.375(14)$ \\
C64 & C65 & $1.409(14)$ \\
C65 & C66 & $1.372(10)$
\end{tabular}

\begin{tabular}{lll}
\hline Atom & Atom & Length/A \\
\hline C67 & C68 & $1.382(10)$ \\
C67 & C72 & $1.423(9)$ \\
C68 & C69 & $1.394(10)$ \\
C69 & C70 & $1.395(12)$ \\
C70 & C71 & $1.371(13)$ \\
C71 & C72 & $1.390(10)$ \\
---- & & \\
11-x,+y,-z; 2 2-x,+y,1-z & \\
\end{tabular}


Table2: Bond Angles in .

\begin{tabular}{|c|c|c|c|c|c|c|c|}
\hline Atom & Atom & Atom & Angle $/^{\circ}$ & Atom & Atom & Atom & Angle $/^{\circ}$ \\
\hline$\overline{01}$ & $\mathrm{Nd1}$ & $01^{1}$ & $146.7(3)$ & $\overline{04}$ & $\mathrm{Nd} 2$ & $04^{2}$ & $147.8(2)$ \\
\hline $01^{1}$ & $\mathrm{Nd} 1$ & 05 & $73.35(13)$ & 04 & $\mathrm{Nd} 2$ & 06 & $73.90(12)$ \\
\hline 01 & $\mathrm{Nd} 1$ & 05 & $73.35(13)$ & $04^{2}$ & $\mathrm{Nd} 2$ & 06 & $73.90(12)$ \\
\hline 01 & $\mathrm{Nd} 1$ & 071 & $115.0(2)$ & 04 & $\mathrm{Nd} 2$ & $010^{2}$ & $130.2(2)$ \\
\hline $01^{1}$ & $\mathrm{Nd} 1$ & 071 & $88.5(2)$ & 04 & $\mathrm{Nd} 2$ & 010 & $75.2(3)$ \\
\hline $01^{1}$ & $\mathrm{Nd} 1$ & 07 & $115.0(2)$ & $04^{2}$ & $\mathrm{Nd} 2$ & $010^{2}$ & $75.2(3)$ \\
\hline 01 & $\mathrm{Nd} 1$ & 07 & $88.5(2)$ & $04^{2}$ & $\mathrm{Nd} 2$ & 010 & $130.2(2)$ \\
\hline 01 & $\mathrm{Nd} 1$ & 091 & $75.2(2)$ & $04^{2}$ & $\mathrm{Nd} 2$ & $011^{2}$ & $72.7(2)$ \\
\hline 01 & $\mathrm{Nd} 1$ & 09 & $134.8(2)$ & $04^{2}$ & $\mathrm{Nd} 2$ & 011 & $137.8(2)$ \\
\hline $01^{1}$ & $\mathrm{Nd} 1$ & 091 & $134.8(2)$ & 04 & $\mathrm{Nd} 2$ & 011 & $72.7(2)$ \\
\hline $01^{1}$ & $\mathrm{Nd} 1$ & 09 & $75.2(2)$ & 04 & $\mathrm{Nd} 2$ & $011^{2}$ & $137.8(2)$ \\
\hline $02^{1}$ & $\mathrm{Nd} 1$ & $01^{1}$ & $83.8(2)$ & 06 & $\mathrm{Nd} 2$ & S3 & $148.07(7)$ \\
\hline 02 & $\mathrm{Nd} 1$ & 01 & $83.8(2)$ & 06 & $\mathrm{Nd} 2$ & $\mathrm{~S}^{2}$ & $148.07(8)$ \\
\hline $02^{1}$ & $\mathrm{Nd} 1$ & 01 & $83.3(2)$ & 06 & $\mathrm{Nd} 2$ & 010 & $134.7(2)$ \\
\hline 02 & Nd1 & $01^{1}$ & $83.3(2)$ & 06 & $\mathrm{Nd} 2$ & $010^{2}$ & $134.7(2)$ \\
\hline $02^{1}$ & $\mathrm{Nd} 1$ & 02 & $134.1(3)$ & $010^{2}$ & $\mathrm{Nd} 2$ & $\mathrm{~S}^{2}$ & $25.9(2)$ \\
\hline $02^{1}$ & $\mathrm{Nd} 1$ & 05 & $67.03(15)$ & 010 & $\mathrm{Nd} 2$ & $\mathrm{~S}^{2}$ & $72.9(2)$ \\
\hline 02 & $\mathrm{Nd} 1$ & 05 & $67.03(15)$ & $010^{2}$ & $\mathrm{Nd} 2$ & S3 & $72.9(2)$ \\
\hline 02 & $\mathrm{Nd} 1$ & 07 & $154.1(2)$ & 010 & $\mathrm{Nd} 2$ & S3 & $25.9(2)$ \\
\hline $02^{1}$ & Nd1 & 071 & $154.1(2)$ & $010^{2}$ & $\mathrm{Nd} 2$ & 010 & $90.7(5)$ \\
\hline $02^{1}$ & $\mathrm{Nd} 1$ & 07 & $69.0(3)$ & 011 & $\mathrm{Nd} 2$ & S3 & $21.6(2)$ \\
\hline 02 & $\mathrm{Nd} 1$ & 071 & $68.9(3)$ & $011^{2}$ & $\mathrm{Nd} 2$ & $\mathrm{~S}^{2}$ & $21.6(2)$ \\
\hline 02 & $\mathrm{Nd} 1$ & 09 & $129.7(3)$ & $011^{2}$ & $\mathrm{Nd} 2$ & S3 & $64.55(19)$ \\
\hline $02^{1}$ & $\mathrm{Nd} 1$ & 091 & $129.7(3)$ & 011 & $\mathrm{Nd} 2$ & $\mathrm{~S} 3^{2}$ & $64.54(19)$ \\
\hline 02 & $\mathrm{Nd} 1$ & 091 & $88.5(2)$ & $011^{2}$ & $\mathrm{Nd} 2$ & 06 & $143.23(19)$ \\
\hline $02^{1}$ & $\mathrm{Nd} 1$ & 09 & $88.5(2)$ & 011 & $\mathrm{Nd} 2$ & 06 & $143.23(19)$ \\
\hline 07 & $\mathrm{Nd} 1$ & 05 & $133.8(2)$ & $011^{2}$ & $\mathrm{Nd} 2$ & $010^{2}$ & $47.3(3)$ \\
\hline 071 & $\mathrm{Nd} 1$ & 05 & $133.8(2)$ & 011 & $\mathrm{Nd} 2$ & $010^{2}$ & $63.4(3)$ \\
\hline 07 & $\mathrm{Nd} 1$ & 071 & $92.4(4)$ & $011^{2}$ & $\mathrm{Nd} 2$ & 010 & $63.4(3)$ \\
\hline 09 & $\mathrm{Nd} 1$ & 05 & $141.74(18)$ & 011 & $\mathrm{Nd} 2$ & 010 & $47.3(3)$ \\
\hline 091 & $\mathrm{Nd} 1$ & 05 & $141.74(18)$ & $011^{2}$ & $\mathrm{Nd} 2$ & 011 & $73.5(4)$ \\
\hline 091 & Nd1 & 07 & $65.6(3)$ & $013^{1}$ & $\mathrm{Cl} 1$ & 013 & $81(2)$ \\
\hline 091 & $\mathrm{Nd} 1$ & 071 & $47.6(3)$ & $013^{1}$ & $\mathrm{Cl} 1$ & 014 & $110.7(11)$ \\
\hline 09 & Nd1 & 07 & $47.6(3)$ & 013 & $\mathrm{Cl} 1$ & 014 & $102.3(11)$ \\
\hline 09 & $\mathrm{Nd} 1$ & 071 & $65.6(3)$ & 013 & $\mathrm{Cl} 1$ & $014^{1}$ & $110.7(11)$ \\
\hline 091 & $\mathrm{Nd} 1$ & 09 & $76.5(4)$ & $013^{1}$ & $\mathrm{Cl} 1$ & $014^{1}$ & $102.3(11)$ \\
\hline $\mathrm{S} 3^{2}$ & $\mathrm{Nd} 2$ & S3 & $63.86(15)$ & 014 & $\mathrm{Cl} 1$ & 0141 & $136.4(18)$ \\
\hline $03^{2}$ & $\mathrm{Nd} 2$ & S3 & $108.40(15)$ & $015^{2}$ & $\mathrm{Cl} 2$ & 015 & $108.1(7)$ \\
\hline $03^{2}$ & $\mathrm{Nd} 2$ & $\mathrm{~S}^{2}$ & $95.26(17)$ & $016^{2}$ & $\mathrm{Cl} 2$ & 015 & $118.2(12)$ \\
\hline 03 & $\mathrm{Nd} 2$ & $\mathrm{~S}^{2}$ & $108.40(15)$ & $016^{2}$ & $\mathrm{Cl} 2$ & $015^{2}$ & $102.2(7)$ \\
\hline 03 & $\mathrm{Nd} 2$ & S3 & $95.26(17)$ & 016 & $\mathrm{Cl} 2$ & $015^{2}$ & $118.2(12)$ \\
\hline 03 & $\mathrm{Nd} 2$ & $03^{2}$ & $152.2(3)$ & 016 & $\mathrm{Cl} 2$ & 015 & $102.2(7)$ \\
\hline 03 & $\mathrm{Nd} 2$ & 04 & $85.92(19)$ & $016^{2}$ & $\mathrm{Cl} 2$ & 016 & $109(2)$ \\
\hline $03^{2}$ & $\mathrm{Nd} 2$ & 04 & $86.4(2)$ & S4 & S5 & $\mathrm{S} 4^{2}$ & $31.6(4)$ \\
\hline $03^{2}$ & $\mathrm{Nd} 2$ & $04^{2}$ & $85.93(19)$ & $\mathrm{S} 4$ & S5 & $010^{2}$ & $105.2(4)$ \\
\hline 03 & $\mathrm{Nd} 2$ & $04^{2}$ & $86.4(2)$ & $010^{2}$ & S5 & $\mathrm{S} 4^{2}$ & $117.1(4)$ \\
\hline 03 & $\mathrm{Nd} 2$ & 06 & $76.11(14)$ & $011^{2}$ & S5 & $\mathrm{S} 4^{2}$ & $101.3(4)$ \\
\hline $\mathrm{O}^{2}$ & $\mathrm{Nd} 2$ & 06 & 76.11(14) & $011^{2}$ & S5 & S4 & $127.8(4)$ \\
\hline 03 & $\mathrm{Nd} 2$ & 010 & $69.4(3)$ & $011^{2}$ & S5 & $010^{2}$ & $72.5(5)$ \\
\hline $03^{2}$ & $\mathrm{Nd} 2$ & 010 & $133.6(2)$ & $012 A^{2}$ & S5 & $\mathrm{S} 4^{2}$ & $99.6(7)$ \\
\hline 03 & $\mathrm{Nd} 2$ & $010^{2}$ & $133.6(2)$ & $012 A^{2}$ & S5 & $\mathrm{S} 4$ & $104.9(7)$ \\
\hline $03^{2}$ & $\mathrm{Nd} 2$ & $010^{2}$ & $69.4(3)$ & $012 A^{2}$ & S5 & $010^{2}$ & 143.1(8) \\
\hline $03^{2}$ & $\mathrm{Nd} 2$ & $011^{2}$ & $116.1(3)$ & $012 A^{2}$ & S5 & $011^{2}$ & $104.5(7)$ \\
\hline 03 & $\mathrm{Nd} 2$ & 011 & $116.1(3)$ & S1 & $\mathrm{S} 2$ & $\mathrm{~S} 1^{1}$ & $101.9(2)$ \\
\hline 03 & $\mathrm{Nd} 2$ & $011^{2}$ & $86.8(3)$ & S5 & S4 & $\mathrm{S} 5^{2}$ & $104.7(3)$ \\
\hline $03^{2}$ & $\mathrm{Nd} 2$ & 011 & $86.8(3)$ & $\mathrm{S} 4^{2}$ & S4 & $\mathrm{S} 5^{2}$ & $45.9(3)$ \\
\hline 04 & $\mathrm{Nd} 2$ & $\mathrm{~S} 3^{2}$ & $137.04(14)$ & $\mathrm{S} 4^{2}$ & S4 & S5 & $102.5(5)$ \\
\hline $04^{2}$ & $\mathrm{Nd} 2$ & S3 & $137.04(14)$ & 07 & $\mathrm{~S} 1$ & $\mathrm{~S} 2$ & $114.9(3)$ \\
\hline 04 & $\mathrm{Nd} 2$ & S3 & $74.87(14)$ & 08 & $\mathrm{~S} 1$ & $\mathrm{~S} 2$ & $107.4(6)$ \\
\hline $04^{2}$ & $\mathrm{Nd} 2$ & $\mathrm{~S} 3^{2}$ & $74.87(14)$ & 08 & S1 & 07 & $125.7(7)$ \\
\hline
\end{tabular}




\begin{tabular}{|c|c|c|c|}
\hline Atom & Atom & Atom & Angle $/^{\circ}$ \\
\hline$\overline{08}$ & S1 & 09 & $113.5(5)$ \\
\hline 09 & $\mathrm{~S} 1$ & $\mathrm{~S} 2$ & $103.4(3)$ \\
\hline 09 & $\mathrm{~S} 1$ & 07 & $88.5(6)$ \\
\hline 010 & S3 & $\mathrm{Nd} 2$ & $53.8(4)$ \\
\hline 011 & S3 & $\mathrm{Nd} 2$ & $47.3(4)$ \\
\hline 011 & S3 & 010 & $100.3(6)$ \\
\hline 011 & S3 & 012 & $124.6(7)$ \\
\hline 012 & S3 & $\mathrm{Nd} 2$ & $150.6(5)$ \\
\hline 012 & S3 & 010 & $117.5(7)$ \\
\hline 01 & $\mathrm{P} 1$ & $\mathrm{C} 1$ & $110.5(3)$ \\
\hline 01 & $\mathrm{P} 1$ & $\mathrm{C} 7$ & $109.6(3)$ \\
\hline 01 & $\mathrm{P} 1$ & C13 & $112.4(3)$ \\
\hline $\mathrm{C} 7$ & $\mathrm{P} 1$ & $\mathrm{C} 1$ & $109.2(3)$ \\
\hline $\mathrm{C} 7$ & $\mathrm{P} 1$ & C13 & $107.4(3)$ \\
\hline C13 & $\mathrm{P} 1$ & $\mathrm{C} 1$ & $107.6(3)$ \\
\hline 02 & P2 & C19 & $110.8(4)$ \\
\hline 02 & $\mathrm{P} 2$ & $\mathrm{C} 25$ & $110.0(4)$ \\
\hline 02 & $\mathrm{P} 2$ & C31 & $112.6(3)$ \\
\hline C19 & $\mathrm{P} 2$ & $\mathrm{C} 25$ & $108.7(4)$ \\
\hline C19 & P2 & C31 & $105.9(4)$ \\
\hline C31 & $\mathrm{P} 2$ & $\mathrm{C} 25$ & $108.7(4)$ \\
\hline 03 & P3 & C37 & $112.7(3)$ \\
\hline 03 & P3 & C43 & $110.3(3)$ \\
\hline 03 & P3 & C49 & $111.1(3)$ \\
\hline C37 & P3 & $\mathrm{C} 43$ & $109.1(3)$ \\
\hline C49 & P3 & C37 & $106.3(3)$ \\
\hline C49 & P3 & C43 & $107.1(3)$ \\
\hline 04 & $\mathrm{P} 4$ & C55 & $113.2(3)$ \\
\hline 04 & $\mathrm{P} 4$ & C61 & $110.3(3)$ \\
\hline 04 & $\mathrm{P} 4$ & C67 & 111.1(3) \\
\hline C55 & $\mathrm{P} 4$ & C61 & $105.9(3)$ \\
\hline C55 & $\mathrm{P} 4$ & C67 & $108.2(3)$ \\
\hline C61 & $\mathrm{P} 4$ & C67 & $107.8(3)$ \\
\hline P1 & 01 & $\mathrm{Nd} 1$ & $163.0(3)$ \\
\hline P2 & 02 & $\mathrm{Nd} 1$ & $158.8(4)$ \\
\hline P3 & 03 & $\mathrm{Nd} 2$ & $174.0(4)$ \\
\hline P4 & 04 & $\mathrm{Nd} 2$ & $166.9(3)$ \\
\hline S1 & 07 & $\mathrm{Nd} 1$ & $101.4(4)$ \\
\hline S1 & 09 & $\mathrm{Nd} 1$ & $115.9(6)$ \\
\hline $\mathrm{S} 5^{2}$ & 010 & $\mathrm{Nd} 2$ & $103.5(4)$ \\
\hline S3 & 010 & $\mathrm{Nd} 2$ & $100.3(5)$ \\
\hline$S 5^{2}$ & 011 & $\mathrm{Nd} 2$ & $124.8(6)$ \\
\hline S3 & 011 & $\mathrm{Nd} 2$ & $111.1(6)$ \\
\hline Cl1 & 013 & $013^{1}$ & $49.7(10)$ \\
\hline $\mathrm{C} 2$ & $\mathrm{C} 1$ & $\mathrm{P} 1$ & $118.6(5)$ \\
\hline $\mathrm{C} 2$ & $\mathrm{C} 1$ & C6 & $118.6(6)$ \\
\hline C6 & $\mathrm{C} 1$ & $\mathrm{P} 1$ & $122.7(5)$ \\
\hline $\mathrm{C} 1$ & $\mathrm{C} 2$ & $\mathrm{C} 3$ & $119.9(7)$ \\
\hline $\mathrm{C} 4$ & $\mathrm{C} 3$ & $\mathrm{C} 2$ & $121.0(7)$ \\
\hline $\mathrm{C} 3$ & $\mathrm{C} 4$ & $\mathrm{C} 5$ & $119.8(7)$ \\
\hline $\mathrm{C} 4$ & $\mathrm{C} 5$ & C6 & $120.3(8)$ \\
\hline C5 & C6 & $\mathrm{C} 1$ & $120.3(7)$ \\
\hline C8 & $\mathrm{C} 7$ & $\mathrm{P} 1$ & $120.2(6)$ \\
\hline C12 & $\mathrm{C} 7$ & $\mathrm{P} 1$ & $120.1(7)$ \\
\hline C12 & $\mathrm{C} 7$ & $\mathrm{C} 8$ & $119.4(8)$ \\
\hline $\mathrm{C} 9$ & $\mathrm{C} 8$ & $\mathrm{C} 7$ & $118.6(10)$ \\
\hline C10 & $\mathrm{C} 9$ & $\mathrm{C} 8$ & $120.6(11)$ \\
\hline C11 & C10 & $\mathrm{C} 9$ & $118.5(10)$ \\
\hline C10 & C11 & C12 & $122.5(13)$ \\
\hline $\mathrm{C} 7$ & $\mathrm{C} 12$ & C11 & 119.1(12) \\
\hline C14 & C13 & $\mathrm{P} 1$ & $118.3(5)$ \\
\hline C18 & C13 & $\mathrm{P} 1$ & $122.2(5)$ \\
\hline
\end{tabular}

\begin{tabular}{|c|c|c|c|}
\hline Atom & Atom & Atom & Angle $/^{\circ}$ \\
\hline$\overline{\mathrm{C} 18}$ & C13 & C14 & $119.1(6)$ \\
\hline C13 & C14 & C15 & $120.2(7)$ \\
\hline C16 & C15 & C14 & $120.2(7)$ \\
\hline C17 & C16 & C15 & $119.8(7)$ \\
\hline C16 & C17 & C18 & $120.4(7)$ \\
\hline C13 & C18 & $\mathrm{C} 17$ & $120.3(6)$ \\
\hline C20 & C19 & P2 & $120.6(9)$ \\
\hline C20 & C19 & $\mathrm{C} 24$ & $118.2(9)$ \\
\hline C24 & C19 & $\mathrm{P} 2$ & $121.2(7)$ \\
\hline C19 & $\mathrm{C} 20$ & $\mathrm{C} 21$ & 121.1(11) \\
\hline $\mathrm{C} 22$ & $\mathrm{C} 21$ & $\mathrm{C} 20$ & $119.7(10)$ \\
\hline C21 & $\mathrm{C} 22$ & $\mathrm{C} 23$ & $119.3(11)$ \\
\hline $\mathrm{C} 24$ & $\mathrm{C} 23$ & $\mathrm{C} 22$ & $121.4(13)$ \\
\hline C23 & $\mathrm{C} 24$ & C19 & $120.2(11)$ \\
\hline $\mathrm{C} 26$ & $\mathrm{C} 25$ & $\mathrm{P} 2$ & $122.2(6)$ \\
\hline C30 & $\mathrm{C} 25$ & $\mathrm{P} 2$ & $118.2(6)$ \\
\hline C30 & $\mathrm{C} 25$ & $\mathrm{C} 26$ & $119.6(7)$ \\
\hline $\mathrm{C} 27$ & $\mathrm{C} 26$ & $\mathrm{C} 25$ & $119.6(8)$ \\
\hline C28 & $\mathrm{C} 27$ & $\mathrm{C} 26$ & $121.2(8)$ \\
\hline $\mathrm{C} 27$ & $\mathrm{C} 28$ & $\mathrm{C} 29$ & $119.0(8)$ \\
\hline C30 & $\mathrm{C} 29$ & $\mathrm{C} 28$ & $119.9(9)$ \\
\hline C29 & C30 & $\mathrm{C} 25$ & $120.7(8)$ \\
\hline C32 & C31 & P2 & $123.3(7)$ \\
\hline C32 & C31 & C36 & $118.0(8)$ \\
\hline C36 & C31 & P2 & $118.7(6)$ \\
\hline C31 & C32 & C33 & $120.6(10)$ \\
\hline C34 & C33 & C32 & $120.5(9)$ \\
\hline C33 & C34 & C35 & $120.3(10)$ \\
\hline C36 & C35 & C34 & $118.9(10)$ \\
\hline C35 & C36 & C31 & $121.6(9)$ \\
\hline C38 & C37 & P3 & $121.9(5)$ \\
\hline C38 & C37 & $\mathrm{C} 42$ & $120.0(6)$ \\
\hline C42 & C37 & P3 & $118.1(5)$ \\
\hline C37 & C38 & C39 & $119.3(7)$ \\
\hline $\mathrm{C} 40$ & C39 & C38 & $121.1(8)$ \\
\hline C39 & $\mathrm{C} 40$ & C41 & $118.9(7)$ \\
\hline C42 & $\mathrm{C} 41$ & $\mathrm{C} 40$ & $120.3(7)$ \\
\hline C41 & $\mathrm{C} 42$ & C37 & $120.5(7)$ \\
\hline C44 & $\mathrm{C} 43$ & P3 & $118.4(5)$ \\
\hline C48 & $\mathrm{C} 43$ & P3 & $121.9(5)$ \\
\hline C48 & $\mathrm{C} 43$ & C44 & $119.7(6)$ \\
\hline $\mathrm{C} 45$ & $\mathrm{C} 44$ & $\mathrm{C} 43$ & $119.6(7)$ \\
\hline $\mathrm{C} 46$ & $\mathrm{C} 45$ & C44 & $120.8(8)$ \\
\hline C45 & $\mathrm{C} 46$ & $\mathrm{C} 47$ & $119.9(7)$ \\
\hline C46 & $\mathrm{C} 47$ & C48 & $120.3(7)$ \\
\hline C43 & $\mathrm{C} 48$ & C47 & $119.8(7)$ \\
\hline C50 & $\mathrm{C} 49$ & P3 & $122.8(5)$ \\
\hline C50 & $\mathrm{C} 49$ & C54 & $118.7(7)$ \\
\hline C54 & C49 & P3 & $118.4(6)$ \\
\hline C51 & C50 & C49 & $121.0(7)$ \\
\hline C52 & C51 & $\mathrm{C} 50$ & $118.5(9)$ \\
\hline C53 & C52 & C51 & $120.9(9)$ \\
\hline C52 & C53 & $\mathrm{C} 54$ & $120.6(8)$ \\
\hline C53 & C54 & C49 & $120.2(9)$ \\
\hline C56 & C55 & $\mathrm{P} 4$ & $121.2(6)$ \\
\hline C60 & C55 & $\mathrm{P} 4$ & $119.9(6)$ \\
\hline C60 & C55 & C56 & $118.8(7)$ \\
\hline C57 & C56 & C55 & $119.4(9)$ \\
\hline C56 & C57 & C58 & $121.1(9)$ \\
\hline C59 & C58 & C57 & $119.8(8)$ \\
\hline C58 & C59 & C60 & $119.7(8)$ \\
\hline C55 & $\mathrm{C} 60$ & C59 & $121.2(7)$ \\
\hline
\end{tabular}




\begin{tabular}{llll}
\hline Atom & Atom & Atom & Angle $^{\circ}$ \\
\hline C62 & C61 & P4 & $122.3(6)$ \\
C66 & C61 & P4 & $118.1(6)$ \\
C66 & C61 & C62 & $119.6(7)$ \\
C63 & C62 & C61 & $119.2(8)$ \\
C64 & C63 & C62 & $120.9(8)$ \\
C63 & C64 & C65 & $119.7(8)$ \\
C66 & C65 & C64 & $119.9(8)$ \\
C65 & C66 & C61 & $120.8(8)$ \\
C68 & C67 & P4 & $122.9(5)$
\end{tabular}

\begin{tabular}{llll}
\hline Atom & Atom & Atom & Angle/ $^{\circ}$ \\
\hline C68 & C67 & C72 & $120.6(6)$ \\
C72 & C67 & P4 & $116.5(5)$ \\
C67 & C68 & C69 & $119.6(7)$ \\
C68 & C69 & C70 & $119.9(8)$ \\
C71 & C70 & C69 & $120.5(7)$ \\
C70 & C71 & C72 & $121.0(7)$ \\
C71 & C72 & C67 & $118.3(7)$ \\
---- & & & \\
${ }^{11-x,+y,-z ;}{ }^{2} 2-\mathrm{x},+y, 1-z$ & &
\end{tabular}




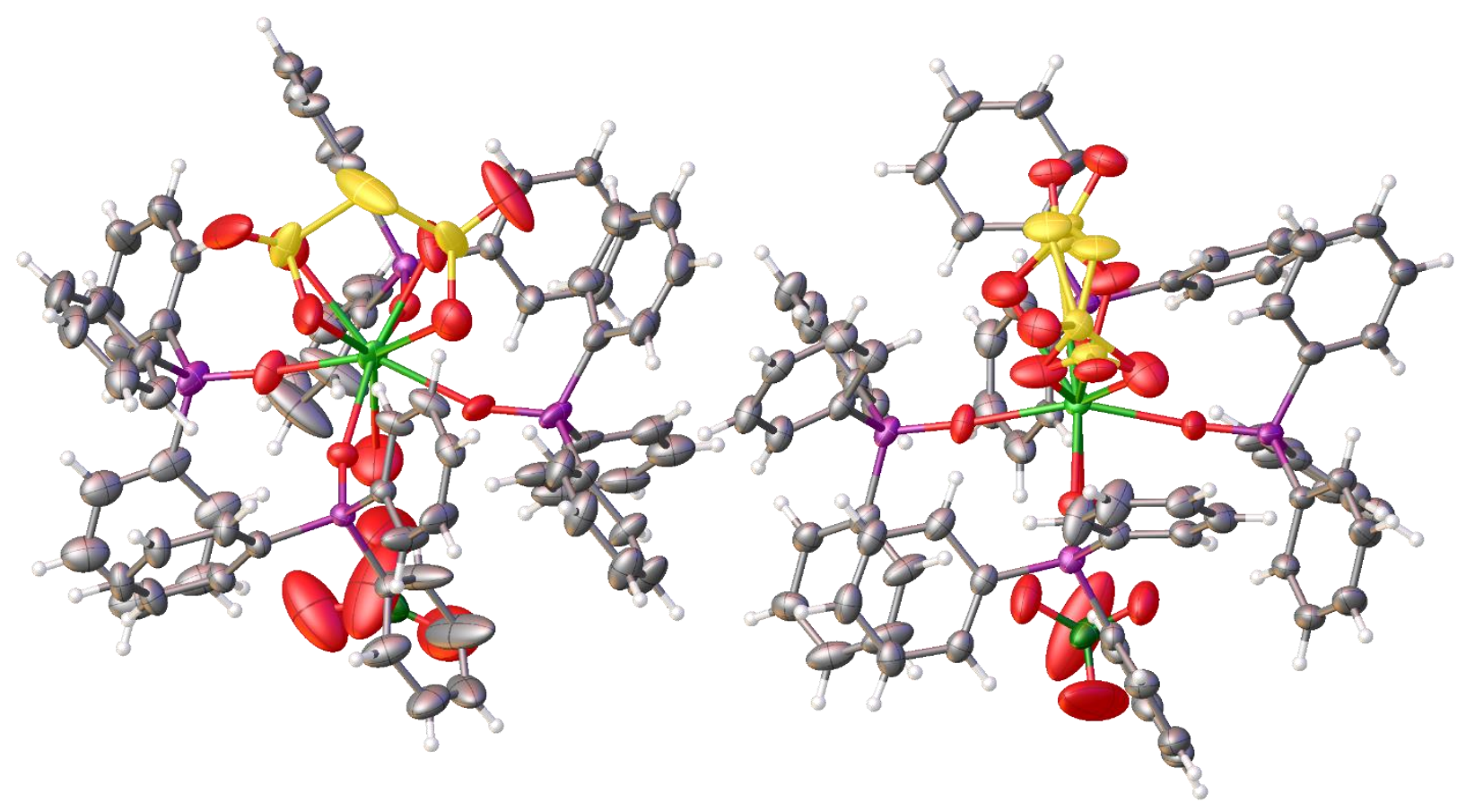




\section{Crystal Data and Experimental $\left[\mathrm{Nd}\left(\kappa^{2}-\mathrm{O}, \mathrm{O}^{\prime}-\mathrm{S}_{4} \mathrm{O}_{6}\right)\left(\mathrm{Ph}_{3} \mathrm{PO}\right)_{4}\left(\mathrm{H}_{2} \mathrm{O}\right)\right] \cdot 3\left[\mathrm{ClO}_{4}\right]$}

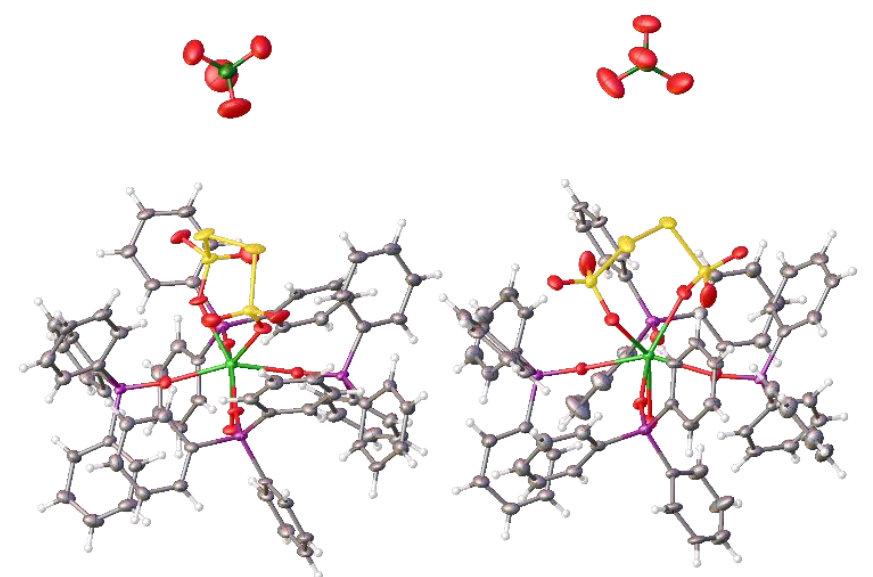

Figure 1: Thermal ellipsoids drawn at the $50 \%$ probability level.

Experimental. A suitable colourless cut shard-shaped crystal $\left(0.220 \times 0.140 \times 0.090 \mathrm{~mm}^{3}\right)$ was selected and mounted on a MITIGEN holder in perfluoroether oil on a Bruker-Nonius FR591 diffractometer equipped with a rotating anode and graphite monochromator and a Bruker-Nonius $95 \mathrm{~mm}$ CCD camera on $\kappa$-goniostat. The crystal was kept at a steady $T=120(2) \mathrm{K}$ during data collection. The structure was solved with the ShelXT (Sheldrick, 2015) structure solution program using the Intrinsic Phasing solution method and by using 0lex2 (Dolomanov et al., 2009) as the graphical interface. The model was refined with version 2014/7 of ShelXL (Sheldrick, 2015) using Least Squares minimisation.

Crystal Data. $\mathrm{C}_{72} \mathrm{H}_{60} \mathrm{ClNdO}_{15} \mathrm{P}_{4} \mathrm{~S}_{4}, \quad M_{r}=1597.01$, monoclinic, $C 2$ (No. 5), a = 20.0936(7) $\AA$, b = 17.3394(7) , $\mathrm{c}=19.6719(7) \AA, \quad \beta=90.081(3)^{\circ}, \quad \alpha=\gamma=90^{\circ}, \quad V=$ 6853.9(4) $\AA^{3}, T=120(2) \mathrm{K}, Z=4, Z^{\prime}=1, \mu\left(\mathrm{MoK}_{\alpha}\right)=1.080$ $\mathrm{mm}^{-1}, 35506$ reflections measured, 15571 unique $\left(R_{\text {int }}=\right.$ 0.0770 ) which were used in all calculations. The final $w R_{2}$ was 0.2016 (all data) and $R_{1}$ was 0.0789 (I > 2(I)).

\begin{tabular}{|c|c|}
\hline Formula & $\mathrm{C}_{72} \mathrm{H}_{60} \mathrm{ClNdO}_{15} \mathrm{P}_{4} \mathrm{~S}_{4}$ \\
\hline$D_{\text {calc. }} / \mathrm{g} \mathrm{cm}^{-3}$ & 1.548 \\
\hline$\mu / \mathrm{mm}^{-1}$ & 1.080 \\
\hline Formula Weight & 1597.01 \\
\hline Colour & colourless \\
\hline Shape & cut shard \\
\hline Size $/ \mathrm{mm}^{3}$ & $0.220 \times 0.140 \times 0.090$ \\
\hline$T / \mathrm{K}$ & $120(2)$ \\
\hline Crystal System & monoclinic \\
\hline Flack Parameter & $0.029(6)$ \\
\hline Hooft Parameter & $0.064(7)$ \\
\hline Space Group & $C 2$ \\
\hline$a / \AA$ & $20.0936(7)$ \\
\hline$b / \AA$ & $17.3394(7)$ \\
\hline$c / \AA$ & $19.6719(7)$ \\
\hline$\alpha /^{\circ}$ & 90 \\
\hline$\beta /^{\circ}$ & $90.081(3)$ \\
\hline$\gamma /^{\circ}$ & 90 \\
\hline $\mathrm{V} / \AA^{3}$ & $6853.9(4)$ \\
\hline$Z$ & 4 \\
\hline$Z^{\prime}$ & 1 \\
\hline Wavelength/Å & 0.71073 \\
\hline Radiation type & $\operatorname{MoK}_{\alpha}$ \\
\hline$\Theta_{\min } /^{\circ}$ & 3.104 \\
\hline$\Theta_{\max } /^{\circ}$ & 27.481 \\
\hline Measured Refl. & 35506 \\
\hline Independent Refl. & 15571 \\
\hline $\begin{array}{l}\text { Reflections with I > } \\
2(\mathrm{I})\end{array}$ & 12518 \\
\hline Rint & 0.0770 \\
\hline Parameters & 877 \\
\hline Restraints & 781 \\
\hline Largest Peak & 5.060 \\
\hline Deepest Hole & -2.450 \\
\hline GooF & 1.084 \\
\hline$w R_{2}$ (all data) & 0.2016 \\
\hline$w R_{2}$ & 0.1904 \\
\hline$R_{1}$ (all data) & 0.1011 \\
\hline$R_{1}$ & 0.0789 \\
\hline
\end{tabular}


Structure Quality Indicators

\section{Reflections: \\ d $\min ($ Mo \\ 0.77 \\ 9.2 \\ Rint $7.70 \%$ \\ complete $\quad 100 \%$ \\ Refinement:

Shift $\quad 0.000$ Max Peak 5.1 Min Peak -2.5 Goof 1.084 Flack 029 (6)

A colourless cut shard-shaped crystal with dimensions $0.220 \times 0.140 \times 0.090 \mathrm{~mm}^{3}$ was mounted on a MITIGEN holder in perfluoroether oil on a Bruker-Nonius FR591 diffractometer equipped with a rotating anode and graphite monochromator and a Bruker-Nonius $95 \mathrm{~mm}$ CCD camera on $\kappa$-goniostat. The crystal was kept at a steady $T=120(2)$ K during data collection.

Data were measured using $\mathrm{MoK}_{\alpha}$ radiation. The maximum resolution that was achieved was $\Theta=27.481^{\circ}$ $(0.77 \AA)$.

The diffraction pattern was indexed and the unit cell was refined on 11302 reflections, $90 \%$ of the observed reflections.

Data reduction, scaling and absorption corrections were performed using DENZO (Otwinowski, 1997). The final completeness is $99.80 \%$ out to $27.481^{\circ}$ in $\Theta$.

An empirical absorption correction was performed using SADABS (Sheldrick, 2003). The absorption coefficient $\mu$ of this material is $1.080 \mathrm{~mm}^{-1}$ at this wavelength $(\lambda=0.711 \AA)$ and the minimum and maximum transmissions are 0.688 and 1.000 .

The structure was solved and the space group C2 (\# 5) determined by the ShelXT (Sheldrick, 2015) structure solution program using Intrinsic Phasing and refined by Least Squares using version 2014/7 of ShelXL (Sheldrick, 2015). All non-hydrogen atoms were refined anisotropically. Hydrogen atom positions were calculated geometrically and refined using the riding model. Some restraints were applied.

There is a 2 half molecules in the asymmetric unit, which is represented by the reported sum formula. In other words: $\mathrm{Z}$ is 4 and $\mathrm{Z}$ ' is 1 .

The Flack parameter was refined to 0.029(6). Determination of absolute structure using Bayesian statistics on Bijvoet differences using the Olex2 results in 0.064(7). Note: The Flack parameter is used to determine chirality of the crystal studied, the value should be near 0 , a value of 1 means that the stereochemistry is wrong and the model should be inverted. A value of 0.5 means that the crystal consists of a racemic mixture of the two enantiomers. 
Reflection Statistics

$\begin{array}{llll}\begin{array}{l}\text { Total reflections (after } \\ \text { filtering) }\end{array} & 35506 & \text { Unique reflections } & 15571 \\ \text { Completeness } & 0.992 & \text { Mean I/ } \sigma & 8.61 \\ \text { hklmax collected } & (26,22,25) & \text { hklmin collected } & (-26,-22,-25) \\ \text { hklmax } \text { used } & (26,22,25) & \text { hklmin } \text { used } & (-26,-22,0) \\ \text { Lim dmax collected } & 7.0 & \text { Lim d } \mathrm{d}_{\min } \text { collected } & 0.77 \\ \mathrm{~d}_{\max } \text { used } & 6.56 & \mathrm{~d}_{\min } \text { used } & 0.77 \\ \text { Friedel pairs } & 11196 & \text { Friedel pairs merged } & 0 \\ \text { Inconsistent equivalents } & 54 & \text { Rint } & 0.077 \\ \text { Rsigma } & 0.1085 & \text { Intensity transformed } & 0 \\ \text { Omitted reflections } & 0 & \text { Omitted by user (OMIT hkl) } & 0 \\ \text { Multiplicity } & (17172,6567,1397,277,2) & \text { Maximum multiplicity } & 8 \\ \text { Removed systematic absences } & 0 & \text { Filtered off (Shel/OMIT) } & 109\end{array}$

Table 1: Bond Lengths in Å.

\begin{tabular}{|c|c|c|c|c|c|}
\hline & \\
\hline Atom & Atom & Length/Å & Atom & Atom & Length/Å \\
\hline$\overline{\mathrm{Nd} 1}$ & 011 & $2.379(9)$ & $\overline{\mathrm{C} 17}$ & C16 & $1.38(2)$ \\
\hline $\mathrm{Nd} 1$ & 01 & $2.379(9)$ & $\mathrm{C} 2$ & $\mathrm{C} 1$ & $1.382(19)$ \\
\hline Nd1 & 03 & $2.535(13)$ & $\mathrm{C} 34$ & C35 & $1.39(2)$ \\
\hline Nd1 & 04 & $2.413(9)$ & $\mathrm{C} 26$ & $\mathrm{C} 25$ & $1.434(19)$ \\
\hline Nd1 & 041 & $2.413(9)$ & $\mathrm{C} 26$ & $\mathrm{C} 27$ & $1.40(2)$ \\
\hline $\mathrm{Nd} 1$ & 02 & $2.308(9)$ & $\mathrm{C} 30$ & $\mathrm{C} 25$ & $1.37(2)$ \\
\hline $\mathrm{Nd} 1$ & $02^{1}$ & $2.308(9)$ & $\mathrm{C} 30$ & $\mathrm{C} 29$ & $1.404(19)$ \\
\hline P1 & 01 & $1.498(9)$ & $\mathrm{C} 10$ & C11 & $1.37(2)$ \\
\hline P1 & $\mathrm{C} 7$ & $1.801(14)$ & C11 & $\mathrm{C} 12$ & $1.38(2)$ \\
\hline P1 & $\mathrm{C} 13$ & $1.820(13)$ & C15 & C16 & $1.40(2)$ \\
\hline $\mathrm{P} 1$ & $\mathrm{C} 1$ & $1.800(14)$ & $\mathrm{C} 5$ & $\mathrm{C} 4$ & $1.40(2)$ \\
\hline S2 & $\mathrm{S} 2^{1}$ & $2.008(8)$ & $\mathrm{C} 27$ & $\mathrm{C} 28$ & $1.36(2)$ \\
\hline S2 & $\mathrm{S} 1$ & $2.123(5)$ & $\mathrm{C} 28$ & $\mathrm{C} 29$ & $1.40(2)$ \\
\hline $\mathrm{P} 2$ & C19 & $1.801(14)$ & $\mathrm{Nd} 2$ & 09 & $2.557(14)$ \\
\hline P2 & 02 & $1.521(9)$ & $\mathrm{Nd} 2$ & 072 & $2.366(9)$ \\
\hline P2 & C31 & $1.787(14)$ & $\mathrm{Nd} 2$ & 07 & $2.365(9)$ \\
\hline $\mathrm{P} 2$ & $\mathrm{C} 25$ & $1.791(14)$ & $\mathrm{Nd} 2$ & $08^{2}$ & $2.339(8)$ \\
\hline S1 & 04 & $1.479(10)$ & $\mathrm{Nd} 2$ & 08 & $2.339(8)$ \\
\hline S1 & 05 & $1.414(10)$ & $\mathrm{Nd} 2$ & $010^{2}$ & $2.448(10)$ \\
\hline S1 & 06 & $1.415(12)$ & $\mathrm{Nd} 2$ & 010 & $2.448(10)$ \\
\hline C19 & $\mathrm{C} 24$ & $1.392(19)$ & S3 & $\mathrm{S}^{2}$ & $2.016(9)$ \\
\hline C19 & $\mathrm{C} 20$ & $1.399(19)$ & S3 & $\mathrm{S} 4$ & $2.117(5)$ \\
\hline C14 & $\mathrm{C} 13$ & $1.387(18)$ & $\mathrm{P} 4$ & $\mathrm{C} 67$ & $1.801(14)$ \\
\hline C14 & $\mathrm{C} 15$ & $1.396(19)$ & $\mathrm{P} 4$ & 08 & $1.512(9)$ \\
\hline C32 & C33 & $1.394(17)$ & $\mathrm{P} 4$ & C55 & $1.799(14)$ \\
\hline C32 & C31 & $1.393(18)$ & $\mathrm{P} 4$ & C61 & $1.804(14)$ \\
\hline C33 & C34 & $1.38(2)$ & P3 & 07 & $1.514(9)$ \\
\hline $\mathrm{C} 7$ & $\mathrm{C} 12$ & $1.400(19)$ & P3 & $\mathrm{C} 43$ & $1.788(14)$ \\
\hline C7 & $\mathrm{C} 8$ & $1.38(2)$ & P3 & $\mathrm{C} 49$ & $1.802(14)$ \\
\hline $\mathrm{C} 9$ & $\mathrm{C} 10$ & $1.40(2)$ & P3 & $\mathrm{C} 37$ & $1.786(14)$ \\
\hline C9 & $\mathrm{C} 8$ & $1.39(2)$ & $\mathrm{S} 4$ & 012 & $1.422(11)$ \\
\hline C3 & $\mathrm{C} 2$ & $1.41(2)$ & $\mathrm{S} 4$ & 010 & $1.475(11)$ \\
\hline $\mathrm{C} 3$ & $\mathrm{C} 4$ & $1.37(2)$ & $\mathrm{S} 4$ & 011 & $1.450(12)$ \\
\hline $\mathrm{C} 24$ & $\mathrm{C} 23$ & $1.39(2)$ & $\mathrm{C} 50$ & C49 & $1.385(19)$ \\
\hline $\mathrm{C} 22$ & $\mathrm{C} 21$ & $1.38(2)$ & $\mathrm{C} 50$ & C51 & $1.398(18)$ \\
\hline $\mathrm{C} 22$ & $\mathrm{C} 23$ & $1.38(2)$ & $\mathrm{C} 43$ & $\mathrm{C} 48$ & $1.411(18)$ \\
\hline C21 & $\mathrm{C} 20$ & $1.40(2)$ & $\mathrm{C} 43$ & $\mathrm{C} 44$ & $1.395(18)$ \\
\hline C6 & $\mathrm{C} 1$ & $1.41(2)$ & C63 & $\mathrm{C} 62$ & $1.385(19)$ \\
\hline C6 & $\mathrm{C} 5$ & $1.39(2)$ & C63 & $\mathrm{C} 64$ & $1.38(2)$ \\
\hline C13 & C18 & $1.387(18)$ & $\mathrm{C} 67$ & $\mathrm{C} 72$ & $1.430(19)$ \\
\hline C36 & C31 & $1.419(18)$ & $\mathrm{C} 67$ & C68 & $1.34(2)$ \\
\hline C36 & $\mathrm{C} 35$ & $1.36(2)$ & C38 & C37 & $1.40(2)$ \\
\hline C17 & C18 & $1.38(2)$ & C38 & C39 & $1.40(2)$ \\
\hline
\end{tabular}




\begin{tabular}{lll}
\hline Atom & Atom & Length/Å \\
\hline C62 & C61 & $1.375(18)$ \\
C55 & C60 & $1.380(19)$ \\
C55 & C56 & $1.411(18)$ \\
C66 & C61 & $1.401(18)$ \\
C66 & C65 & $1.391(19)$ \\
C54 & C49 & $1.39(2)$ \\
C54 & C53 & $1.40(2)$ \\
C60 & C59 & $1.388(19)$ \\
C42 & C41 & $1.39(2)$ \\
C42 & C37 & $1.41(2)$ \\
C71 & C72 & $1.39(2)$ \\
C71 & C70 & $1.33(2)$ \\
C56 & C57 & $1.36(2)$ \\
C51 & C52 & $1.36(2)$ \\
C47 & C48 & $1.39(2)$ \\
C47 & C46 & $1.39(2)$ \\
C46 & C45 & $1.39(2)$ \\
C41 & C40 & $1.40(2)$
\end{tabular}

\begin{tabular}{lll}
\hline Atom & Atom & Length/Å \\
\hline $\mathrm{C} 70$ & $\mathrm{C} 69$ & $1.37(3)$ \\
$\mathrm{C} 44$ & $\mathrm{C} 45$ & $1.39(2)$ \\
$\mathrm{C} 52$ & $\mathrm{C} 53$ & $1.41(2)$ \\
$\mathrm{C} 40$ & $\mathrm{C} 39$ & $1.36(2)$ \\
$\mathrm{C} 57$ & $\mathrm{C} 58$ & $1.38(2)$ \\
$\mathrm{C} 58$ & $\mathrm{C} 59$ & $1.40(2)$ \\
$\mathrm{C} 64$ & $\mathrm{C} 65$ & $1.38(2)$ \\
$\mathrm{C} 68$ & $\mathrm{C} 69$ & $1.39(2)$ \\
$\mathrm{Cl} 1$ & 014 & $1.411(15)$ \\
$\mathrm{Cl} 1$ & 0141 & $1.411(15)$ \\
$\mathrm{Cl} 1$ & $013^{1}$ & $1.407(18)$ \\
$\mathrm{Cl} 1$ & 013 & $1.407(18)$ \\
$\mathrm{Cl} 2$ & $016^{2}$ & $1.457(16)$ \\
$\mathrm{Cl} 2$ & 016 & $1.457(16)$ \\
$\mathrm{Cl} 2$ & $015^{2}$ & $1.392(17)$ \\
$\mathrm{Cl} 2$ & 015 & $1.392(17)$ \\
---- & & \\
1 -x,+y,1-z; & $21-\mathrm{x},+\mathrm{y},-\mathrm{z}$ &
\end{tabular}

Table 2: Bond Angles in ${ }^{\circ}$

\begin{tabular}{|c|c|c|c|}
\hline Atom & Atom & Atom & Angle $/{ }^{\circ}$ \\
\hline$\overline{\mathrm{O} 1^{1}}$ & $\mathrm{Nd1}$ & 01 & $153.8(4)$ \\
\hline $01^{1}$ & $\mathrm{Nd} 1$ & 03 & $76.9(2)$ \\
\hline 01 & $\mathrm{Nd} 1$ & 03 & $76.9(2)$ \\
\hline $01^{1}$ & $\mathrm{Nd} 1$ & 04 & $127.0(3)$ \\
\hline 01 & Nd1 & 041 & $127.0(3)$ \\
\hline 01 & $\mathrm{Nd} 1$ & 04 & $76.1(3)$ \\
\hline $01^{1}$ & $\mathrm{Nd} 1$ & $04^{1}$ & $76.1(3)$ \\
\hline 04 & $\mathrm{Nd} 1$ & 03 & $143.0(2)$ \\
\hline 041 & $\mathrm{Nd} 1$ & 03 & $143.0(2)$ \\
\hline 04 & $\mathrm{Nd} 1$ & $04^{1}$ & $74.1(5)$ \\
\hline $02^{1}$ & $\mathrm{Nd} 1$ & $01^{1}$ & $83.4(3)$ \\
\hline 02 & $\mathrm{Nd} 1$ & 01 & $83.4(3)$ \\
\hline 02 & $\mathrm{Nd} 1$ & $01^{1}$ & $90.7(3)$ \\
\hline $02^{1}$ & $\mathrm{Nd} 1$ & 01 & $90.7(3)$ \\
\hline $02^{1}$ & $\mathrm{Nd} 1$ & 03 & $77.0(2)$ \\
\hline 02 & $\mathrm{Nd} 1$ & 03 & $77.0(2)$ \\
\hline 02 & $\mathrm{Nd} 1$ & 04 & $124.1(3)$ \\
\hline $02^{1}$ & $\mathrm{Nd} 1$ & 041 & $124.1(3)$ \\
\hline $02^{1}$ & $\mathrm{Nd} 1$ & 04 & $78.4(3)$ \\
\hline 02 & Nd1 & $04^{1}$ & $78.4(3)$ \\
\hline $02^{1}$ & $\mathrm{Nd} 1$ & 02 & $154.0(4)$ \\
\hline 01 & $\mathrm{P} 1$ & $\mathrm{C} 7$ & $114.5(6)$ \\
\hline 01 & $\mathrm{P} 1$ & C13 & $108.7(6)$ \\
\hline 01 & $\mathrm{P} 1$ & $\mathrm{C} 1$ & $110.5(6)$ \\
\hline $\mathrm{C} 7$ & $\mathrm{P} 1$ & C13 & $108.7(6)$ \\
\hline $\mathrm{C} 1$ & $\mathrm{P} 1$ & $\mathrm{C} 7$ & $105.5(6)$ \\
\hline $\mathrm{C} 1$ & P1 & C13 & $108.8(6)$ \\
\hline $\mathrm{S} 2^{1}$ & $\mathrm{~S} 2$ & $\mathrm{~S} 1$ & $104.2(2)$ \\
\hline 02 & $\mathrm{P} 2$ & C19 & $110.0(6)$ \\
\hline 02 & $\mathrm{P} 2$ & C31 & $112.8(6)$ \\
\hline 02 & $\mathrm{P} 2$ & $\mathrm{C} 25$ & $110.2(6)$ \\
\hline C31 & $\mathrm{P} 2$ & C19 & $106.5(6)$ \\
\hline C31 & $\mathrm{P} 2$ & $\mathrm{C} 25$ & $109.6(6)$ \\
\hline $\mathrm{C} 25$ & $\mathrm{P} 2$ & C19 & $107.5(6)$ \\
\hline 04 & $\mathrm{~S} 1$ & $\mathrm{~S} 2$ & $103.9(4)$ \\
\hline 05 & $\mathrm{~S} 1$ & $\mathrm{~S} 2$ & $101.5(5)$ \\
\hline 05 & $\mathrm{~S} 1$ & 04 & $112.5(7)$ \\
\hline 05 & $\mathrm{~S} 1$ & 06 & $117.0(8)$ \\
\hline 06 & $\mathrm{~S} 1$ & $\mathrm{~S} 2$ & $109.3(5)$ \\
\hline
\end{tabular}

\begin{tabular}{llll}
\hline Atom & Atom & Atom & Angle ${ }^{\circ}$ \\
\hline O6 & S1 & O4 & $111.3(7)$ \\
P1 & 01 & Nd1 & $171.2(6)$ \\
S1 & O4 & Nd1 & $137.3(6)$ \\
C24 & C19 & P2 & $118.0(10)$ \\
C24 & C19 & C20 & $120.0(13)$ \\
C20 & C19 & P2 & $122.0(11)$ \\
C13 & C14 & C15 & $118.8(13)$ \\
C31 & C32 & C33 & $121.3(13)$ \\
C34 & C33 & C32 & $118.6(14)$ \\
C12 & C7 & P1 & $121.3(11)$ \\
C8 & C7 & P1 & $119.1(10)$ \\
C8 & C7 & C12 & $119.6(13)$ \\
C8 & C9 & C10 & $119.9(14)$ \\
C4 & C3 & C2 & $120.4(15)$ \\
P2 & O2 & Nd1 & $172.6(6)$ \\
C23 & C24 & C19 & $119.2(14)$ \\
C23 & C22 & C21 & $121.3(15)$ \\
C22 & C21 & C20 & $118.5(14)$ \\
C5 & C6 & C1 & $120.3(14)$ \\
C14 & C13 & P1 & $117.2(10)$ \\
C14 & C13 & C18 & $121.3(12)$ \\
C18 & C13 & P1 & $121.5(10)$ \\
C35 & C36 & C31 & $120.3(13)$ \\
C18 & C17 & C16 & $119.4(14)$ \\
C17 & C18 & C13 & $119.9(13)$ \\
C1 & C2 & C3 & $119.5(15)$ \\
C6 & C1 & P1 & $121.2(10)$ \\
C2 & C1 & P1 & $119.0(11)$ \\
C2 & C1 & C6 & $119.8(13)$ \\
C33 & C34 & C35 & $121.2(15)$ \\
C32 & C31 & P2 & $122.3(10)$ \\
C32 & C31 & C36 & $118.3(13)$ \\
C36 & C31 & P2 & $119.3(10)$ \\
C27 & C26 & C25 & $118.1(14)$ \\
C25 & C30 & C29 & $120.4(14)$ \\
C11 & C10 & C9 & $119.0(14)$ \\
C10 & C11 & C12 & $121.8(15)$ \\
C15 & C16 & $119.6(13)$ \\
C5 & C4 & $119.4(16)$
\end{tabular}




\begin{tabular}{|c|c|c|c|}
\hline Atom & Atom & Atom & Angle $/{ }^{\circ}$ \\
\hline$\overline{\mathrm{C} 11}$ & C12 & C7 & 119.1(14) \\
\hline C17 & C16 & C15 & $120.9(14)$ \\
\hline C26 & $\mathrm{C} 25$ & $\mathrm{P} 2$ & $117.3(11)$ \\
\hline C30 & $\mathrm{C} 25$ & $\mathrm{P} 2$ & $123.0(11)$ \\
\hline C30 & $\mathrm{C} 25$ & $\mathrm{C} 26$ & $119.7(13)$ \\
\hline C28 & $\mathrm{C} 27$ & $\mathrm{C} 26$ & $121.9(14)$ \\
\hline $\mathrm{C} 27$ & $\mathrm{C} 28$ & $\mathrm{C} 29$ & $119.9(13)$ \\
\hline C22 & $\mathrm{C} 23$ & $\mathrm{C} 24$ & $120.6(15)$ \\
\hline C36 & C35 & C34 & $120.2(13)$ \\
\hline C28 & $\mathrm{C} 29$ & C30 & $119.8(14)$ \\
\hline C19 & $\mathrm{C} 20$ & $\mathrm{C} 21$ & $120.3(14)$ \\
\hline C7 & $\mathrm{C} 8$ & $\mathrm{C} 9$ & $120.6(13)$ \\
\hline 07 & $\mathrm{Nd} 2$ & 09 & $74.2(2)$ \\
\hline $07^{2}$ & $\mathrm{Nd} 2$ & 09 & $74.2(2)$ \\
\hline 07 & $\mathrm{Nd} 2$ & $07^{2}$ & $148.4(4)$ \\
\hline $07^{2}$ & $\mathrm{Nd} 2$ & $010^{2}$ & $77.5(3)$ \\
\hline 07 & $\mathrm{Nd} 2$ & $010^{2}$ & $130.1(3)$ \\
\hline $07^{2}$ & $\mathrm{Nd} 2$ & 010 & $130.1(3)$ \\
\hline 07 & $\mathrm{Nd} 2$ & 010 & $77.5(3)$ \\
\hline $08^{2}$ & $\mathrm{Nd} 2$ & 09 & $75.5(2)$ \\
\hline 08 & $\mathrm{Nd} 2$ & 09 & $75.5(2)$ \\
\hline $08^{2}$ & $\mathrm{Nd} 2$ & $07^{2}$ & $85.8(3)$ \\
\hline $08^{2}$ & $\mathrm{Nd} 2$ & 07 & $86.3(3)$ \\
\hline 08 & $\mathrm{Nd} 2$ & 07 & $85.8(3)$ \\
\hline 08 & $\mathrm{Nd} 2$ & $07^{2}$ & $86.3(3)$ \\
\hline 08 & $\mathrm{Nd} 2$ & $08^{2}$ & $151.0(5)$ \\
\hline 08 & $\mathrm{Nd} 2$ & 010 & $77.2(3)$ \\
\hline 08 & $\mathrm{Nd} 2$ & $010^{2}$ & $128.0(4)$ \\
\hline $08^{2}$ & $\mathrm{Nd} 2$ & $010^{2}$ & $77.2(3)$ \\
\hline $08^{2}$ & $\mathrm{Nd} 2$ & 010 & $128.0(4)$ \\
\hline $010^{2}$ & $\mathrm{Nd} 2$ & 09 & $141.6(2)$ \\
\hline 010 & $\mathrm{Nd} 2$ & 09 & $141.6(2)$ \\
\hline 010 & $\mathrm{Nd} 2$ & $010^{2}$ & $76.7(5)$ \\
\hline $\mathrm{S} 3^{2}$ & S3 & $\mathrm{S} 4$ & $104.4(3)$ \\
\hline C67 & $\mathrm{P} 4$ & C61 & $108.4(6)$ \\
\hline 08 & $\mathrm{P} 4$ & C67 & $110.3(6)$ \\
\hline 08 & $\mathrm{P} 4$ & C55 & $111.5(6)$ \\
\hline 08 & $\mathrm{P} 4$ & C61 & $110.6(6)$ \\
\hline C55 & $\mathrm{P} 4$ & C67 & $106.9(6)$ \\
\hline C55 & $\mathrm{P} 4$ & C61 & $109.0(6)$ \\
\hline 07 & P3 & C43 & $110.4(6)$ \\
\hline 07 & P3 & C49 & $110.7(6)$ \\
\hline 07 & P3 & C37 & $113.6(6)$ \\
\hline C43 & P3 & C49 & $109.2(6)$ \\
\hline C37 & P3 & C43 & $108.7(7)$ \\
\hline C37 & P3 & C49 & $104.1(6)$ \\
\hline 012 & $\mathrm{~S} 4$ & S3 & $100.9(4)$ \\
\hline 012 & $\mathrm{~S} 4$ & 010 & $112.2(6)$ \\
\hline 012 & S4 & 011 & $115.9(7)$ \\
\hline 010 & S4 & S3 & $104.1(5)$ \\
\hline 011 & S4 & S3 & $108.9(6)$ \\
\hline 011 & S4 & 010 & $113.2(8)$ \\
\hline P3 & 07 & $\mathrm{Nd} 2$ & $166.0(6)$ \\
\hline C49 & $\mathrm{C} 50$ & C51 & $120.7(14)$ \\
\hline C48 & $\mathrm{C} 43$ & P3 & $118.3(10)$ \\
\hline C44 & $\mathrm{C} 43$ & P3 & $122.0(11)$ \\
\hline C44 & $\mathrm{C} 43$ & C48 & $119.7(13)$ \\
\hline C64 & C63 & C62 & $120.7(13)$ \\
\hline C72 & C67 & $\mathrm{P} 4$ & $122.0(11)$ \\
\hline C68 & C67 & $\mathrm{P} 4$ & $119.0(11)$ \\
\hline C68 & C67 & C72 & $118.5(13)$ \\
\hline P4 & 08 & $\mathrm{Nd} 2$ & $164.9(6)$ \\
\hline
\end{tabular}

\begin{tabular}{|c|c|c|c|}
\hline Atom & Atom & Atom & Angle ${ }^{\circ}$ \\
\hline$\overline{\text { C39 }}$ & C38 & C37 & $119.0(16)$ \\
\hline C61 & C62 & C63 & $120.3(14)$ \\
\hline C60 & C55 & $\mathrm{P} 4$ & $122.7(11)$ \\
\hline C60 & C55 & C56 & $118.2(13)$ \\
\hline C56 & C55 & $\mathrm{P} 4$ & $118.9(10)$ \\
\hline C65 & C66 & C61 & $119.6(13)$ \\
\hline S4 & 010 & $\mathrm{Nd} 2$ & $133.0(7)$ \\
\hline C49 & C54 & C53 & $120.5(13)$ \\
\hline C55 & C60 & C59 & $121.6(14)$ \\
\hline C41 & C42 & C37 & $120.0(14)$ \\
\hline C50 & C49 & P3 & $119.9(11)$ \\
\hline C50 & C49 & C54 & $119.1(13)$ \\
\hline C54 & C49 & P3 & $120.9(10)$ \\
\hline $\mathrm{C} 70$ & C71 & C72 & $119.5(15)$ \\
\hline C57 & C56 & C55 & $120.5(13)$ \\
\hline C52 & C51 & C50 & $120.3(13)$ \\
\hline C48 & $\mathrm{C} 47$ & C46 & $120.0(15)$ \\
\hline $\mathrm{C} 47$ & C48 & C43 & $119.6(14)$ \\
\hline $\mathrm{C} 47$ & C46 & $\mathrm{C} 45$ & $121.0(16)$ \\
\hline $\mathrm{C} 42$ & C41 & $\mathrm{C} 40$ & $119.1(16)$ \\
\hline C62 & C61 & $\mathrm{P} 4$ & $118.8(11)$ \\
\hline C62 & C61 & C66 & $119.6(13)$ \\
\hline C66 & C61 & $\mathrm{P} 4$ & $121.5(10)$ \\
\hline C71 & C72 & C67 & $119.5(15)$ \\
\hline C71 & C70 & C69 & $122.4(16)$ \\
\hline C45 & C44 & $\mathrm{C} 43$ & $120.6(14)$ \\
\hline C38 & C37 & P3 & $123.1(12)$ \\
\hline C38 & C37 & $\mathrm{C} 42$ & $119.8(14)$ \\
\hline $\mathrm{C} 42$ & C37 & P3 & $117.1(11)$ \\
\hline C51 & C52 & C53 & $120.2(13)$ \\
\hline C39 & $\mathrm{C} 40$ & C41 & $121.5(15)$ \\
\hline C56 & C57 & C58 & $120.9(15)$ \\
\hline C54 & C53 & C52 & $119.1(14)$ \\
\hline C57 & C58 & C59 & $120.0(15)$ \\
\hline C65 & C64 & C63 & $119.3(13)$ \\
\hline C40 & C39 & C38 & $120.6(15)$ \\
\hline C67 & C68 & C69 & $121.5(16)$ \\
\hline C60 & C59 & C58 & $118.8(15)$ \\
\hline C46 & $\mathrm{C} 45$ & C44 & $119.0(15)$ \\
\hline $014^{1}$ & $\mathrm{Cl} 1$ & 014 & $109.1(14)$ \\
\hline 013 & $\mathrm{Cl} 1$ & 014 & $111.7(12)$ \\
\hline $013^{1}$ & $\mathrm{Cl} 1$ & 014 & $107.3(12)$ \\
\hline $013^{1}$ & $\mathrm{Cl} 1$ & 0141 & $111.7(12)$ \\
\hline 013 & $\mathrm{Cl} 1$ & $014^{1}$ & $107.3(12)$ \\
\hline 013 & $\mathrm{Cl} 1$ & $013^{1}$ & $110(2)$ \\
\hline 016 & $\mathrm{Cl} 2$ & $016^{2}$ & $105.6(13)$ \\
\hline 015 & $\mathrm{Cl} 2$ & 016 & 108.1(10) \\
\hline $015^{2}$ & $\mathrm{Cl} 2$ & 016 & $110.2(12)$ \\
\hline $015^{2}$ & $\mathrm{Cl} 2$ & $016^{2}$ & 108.1(10) \\
\hline 015 & $\mathrm{Cl} 2$ & $016^{2}$ & $110.2(12)$ \\
\hline 015 & $\mathrm{Cl} 2$ & $015^{2}$ & $114.2(18)$ \\
\hline $\mathrm{C} 3$ & $\mathrm{C} 4$ & $\mathrm{C} 5$ & $120.5(14)$ \\
\hline C64 & C65 & C66 & $120.5(14)$ \\
\hline $\mathrm{C} 70$ & C69 & C68 & $118.4(18)$ \\
\hline
\end{tabular}



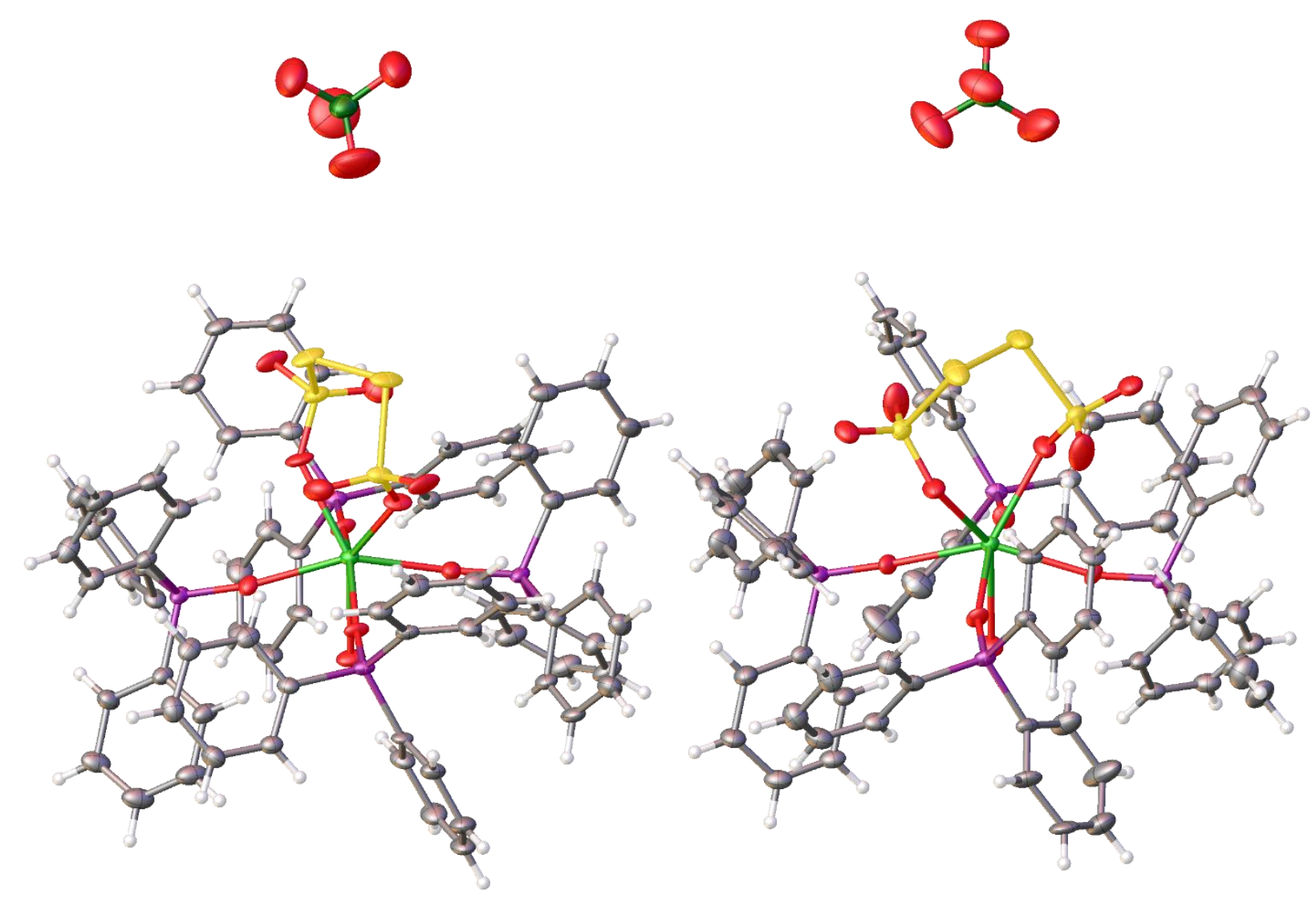


\section{Crystal Data and Experimental $\left[\operatorname{Er}\left(\mathrm{Ph}_{3} \mathrm{PO}\right)_{2}\left(\mathrm{H}_{2} \mathrm{O}\right)_{5}\right] \cdot 5\left(\mathrm{Ph}_{3} \mathrm{PO}\right) \cdot 3\left[\mathrm{ClO}_{4}\right]$}

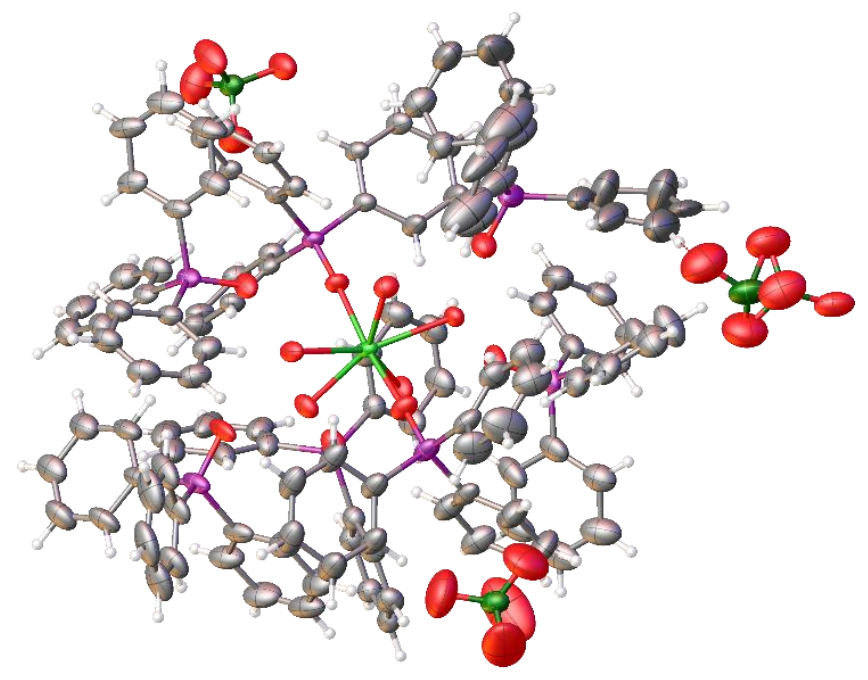

Figure 1: Thermal ellipsoids drawn at the $50 \%$ probability level.

Experimental. A suitable colourless block-shaped crystal $\left(0.120 \times 0.080 \times 0.040 \mathrm{~mm}^{3}\right)$ was selected and mounted on a suitable support on the Daresbury SRS station 9.8 diffractometer. The crystal was kept at a steady $T=$ 120(2) K during data collection. The structure was solved with the ShelXT (Sheldrick, 2015) structure solution program using the Intrinsic Phasing solution method and by using 0lex2 (Dolomanov et al., 2009) as the graphical interface. The model was refined with version 2014/7 of ShelXL (Sheldrick, 2015) using Least Squares minimisation.

Crystal Data. $\mathrm{C}_{126} \mathrm{H}_{105} \mathrm{Cl}_{2.5} \mathrm{ErO}_{22} \mathrm{P}_{7}, \quad M_{r}=2443.77$, orthorhombic, Pbcn (No. 60), $\mathrm{a}=43.152(15) \AA, \mathrm{b}=$ 23.773(8) $\AA, \quad \mathrm{c}=23.799(8) \AA, \quad \alpha=\beta=\gamma=90^{\circ}, \quad V=$ 24414(14) $\AA^{3}, T=120(2) \mathrm{K}, Z=8, Z^{\prime}=1, \mu=0.844 \mathrm{~mm}^{-1}$, 174628 reflections measured, 21554 unique $\left(R_{\text {int }}=\right.$ 0.0711 ) which were used in all calculations. The final $w R_{2}$ was 0.1731 (all data) and $R_{1}$ was 0.0781 (I > 2(I)).

\begin{tabular}{|c|c|}
\hline Formula & $\mathrm{C}_{126} \mathrm{H}_{105} \mathrm{Cl}_{2.5} \mathrm{ErO}_{22} \mathrm{P}_{7}$ \\
\hline$D_{\text {calc. }} / \mathrm{g} \mathrm{cm}^{-3}$ & 1.330 \\
\hline$\mu / \mathrm{mm}^{-1}$ & 0.844 \\
\hline Formula Weight & 2443.77 \\
\hline Colour & colourless \\
\hline Shape & block \\
\hline Size $/ \mathrm{mm}^{3}$ & $0.120 \times 0.080 \times 0.040$ \\
\hline$T / \mathrm{K}$ & $120(2)$ \\
\hline Crystal System & orthorhombic \\
\hline Space Group & Pbcn \\
\hline$a / \AA ̊$ & $43.152(15)$ \\
\hline$b / \AA$ & $23.773(8)$ \\
\hline$c / \AA ̊$ & $23.799(8)$ \\
\hline$\left.\alpha\right|^{\circ}$ & 90 \\
\hline$\beta /^{\circ}$ & 90 \\
\hline$\gamma /^{\circ}$ & 90 \\
\hline $\mathrm{V} / \AA^{3}$ & $24414(14)$ \\
\hline$Z$ & 8 \\
\hline$Z^{\prime}$ & 1 \\
\hline Wavelength/Å & 0.6911 \\
\hline Radiation type & $?$ \\
\hline$\Theta_{\min } /^{\circ}$ & 2.181 \\
\hline$\Theta_{\max } /^{\circ}$ & 24.291 \\
\hline Measured Refl. & 174628 \\
\hline Independent Refl. & 21554 \\
\hline $\begin{array}{l}\text { Reflections with I > } \\
2(\mathrm{I})\end{array}$ & 18416 \\
\hline$R_{\text {int }}$ & 0.0711 \\
\hline Parameters & 1447 \\
\hline Restraints & 1273 \\
\hline Largest Peak & 1.460 \\
\hline Deepest Hole & -2.420 \\
\hline GooF & 1.200 \\
\hline$w R_{2}$ (all data) & 0.1731 \\
\hline$w R_{2}$ & 0.1675 \\
\hline$R_{1}$ (all data) & 0.0894 \\
\hline$R_{1}$ & 0.0781 \\
\hline
\end{tabular}




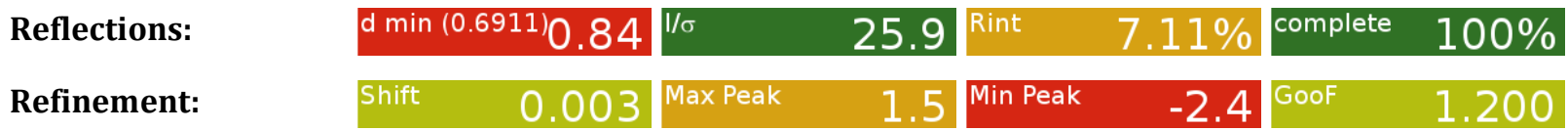

A colourless block-shaped crystal with dimensions $0.120 \times 0.080 \times 0.040 \mathrm{~mm}^{3}$ was mounted on a suitable support. Data were collected using the Daresbury SRS station 9.8 diffractometer, equipped with a Si(111) monochromator and Bruker SMART APEX2 CCD detector (Bruker, 2004), and operating at $T=120(2) \mathrm{K}$.

Data were measured using synchrotron radiation at a wavelength of $0.6911 \AA$ A The maximum resolution that was achieved was $\Theta=24.291^{\circ}(0.84 \AA$ ) .

The diffraction pattern was indexed using SAINT (Bruker, 2004) and the unit cell was refined on 977 reflections, $5 \%$ of the observed reflections.

Data reduction, scaling and absorption corrections were performed using Bruker SAINT. The final completeness is $99.80 \%$ out to $24.291^{\circ}$ in $\Theta$. An empirical absorption correction was performed using SADABS (Bruker, 2004). The absorption coefficient $\mu$ of this material is $0.844 \mathrm{~mm}^{-1}$ at this wavelength $(\lambda=$ $0.6911 \AA$ ) and the minimum and maximum transmissions are 1.000 and 0.860 .

The structure was solved and the space group Pbcn (\# 60) determined by the ShelXT (Sheldrick, 2015) structure solution program using Intrinsic Phasing and refined by Least Squares using version 2014/7 of ShelXL (Sheldrick, 2015). All non-hydrogen atoms were refined anisotropically. Hydrogen atom positions were calculated geometrically and refined using the riding model. There are some small cavities available for solvents. No solvents were identified, possibly some water molecules, so the structure was SQUEEZEd.

There is a single molecule in the asymmetric unit, which is represented by the reported sum formula. In other words: $\mathrm{Z}$ is 8 and $\mathrm{Z}^{\prime}$ is 1 .

\section{Reflection Statistics}

$\begin{array}{ll}\begin{array}{l}\text { Total reflections (after } \\ \text { filtering) }\end{array} & 181102 \\ \text { Completeness } & 0.999 \\ \text { hkl max }_{\text {max }} \text { collected } & (51,28,28) \\ \text { hklmax used } & (51,28,28) \\ \text { Lim dmax collected } & 10.0 \\ \text { d }_{\max } \text { used } & 9.16 \\ \text { Friedel pairs } & 71278 \\ \text { Inconsistent equivalents } & 0 \\ \text { Rsigma }_{\text {Omitted reflections }} & 0.0386 \\ \text { Multiplicity } & 0 \\ \text { Removed systematic absences } & 6474\end{array}$

$\begin{array}{ll}\text { Unique reflections } & 21554 \\ \text { Mean I/ } \sigma & 17.04 \\ \text { hklmin collected }_{\text {hklmin used }} & (-51,-28,-28) \\ \text { Lim dmin collected } & 0.83 \\ \mathrm{~d}_{\text {min }} \text { used } & 0.84 \\ \text { Friedel pairs merged } & 1 \\ \text { Rint }_{\text {Intensity transformed }} & 0.0711 \\ \text { Omitted by user (OMIT hkl) } & 0 \\ \text { Maximum multiplicity } & 12 \\ \text { Filtered off (Shel/OMIT) } & 0\end{array}$

Table 1: Bond Lengths in $\AA$.

\begin{tabular}{lll}
\hline Atom & Atom & Length/Å \\
\hline Er1 & 01 & $2.170(5)$ \\
Er1 & 02 & $2.188(5)$ \\
Er1 & 08 & $2.344(5)$ \\
Er1 & 09 & $2.337(5)$ \\
Er1 & 010 & $2.320(5)$ \\
Er1 & 011 & $2.312(5)$ \\
Er1 & 012 & $2.374(4)$ \\
P1 & 01 & $1.511(5)$
\end{tabular}

\begin{tabular}{lll}
\hline Atom & Atom & Length/Å \\
\hline P1 & C1 & $1.784(10)$ \\
P1 & C7 & $1.796(8)$ \\
P1 & C13 & $1.788(8)$ \\
P2 & O2 & $1.502(5)$ \\
P2 & C19 & $1.802(7)$ \\
P2 & C25 & $1.782(8)$ \\
P2 & C31 & $1.798(7)$ \\
C1 & C2 & $1.383(13)$
\end{tabular}




\begin{tabular}{|c|c|c|}
\hline Atom & Atom & Length/Å \\
\hline$\overline{\mathrm{C} 1}$ & C6 & $1.398(12)$ \\
\hline $\mathrm{C} 2$ & $\mathrm{C} 3$ & $1.363(14)$ \\
\hline C3 & $\mathrm{C} 4$ & $1.408(15)$ \\
\hline $\mathrm{C} 4$ & $\mathrm{C} 5$ & $1.390(17)$ \\
\hline $\mathrm{C} 5$ & $\mathrm{C} 6$ & $1.363(16)$ \\
\hline $\mathrm{C} 7$ & $\mathrm{C} 8$ & $1.397(12)$ \\
\hline $\mathrm{C} 7$ & C12 & $1.376(11)$ \\
\hline $\mathrm{C} 8$ & $\mathrm{C} 9$ & $1.384(12)$ \\
\hline C9 & C10 & $1.399(13)$ \\
\hline C10 & C11 & $1.372(14)$ \\
\hline C11 & C12 & $1.360(12)$ \\
\hline C13 & C14 & $1.369(12)$ \\
\hline C13 & C18 & $1.393(13)$ \\
\hline C14 & C15 & $1.402(14)$ \\
\hline C15 & C16 & $1.396(15)$ \\
\hline C16 & C17 & $1.365(14)$ \\
\hline C17 & C18 & $1.384(12)$ \\
\hline C19 & $\mathrm{C} 20$ & $1.393(10)$ \\
\hline C19 & $\mathrm{C} 24$ & $1.398(10)$ \\
\hline C20 & $\mathrm{C} 21$ & $1.390(10)$ \\
\hline C21 & $\mathrm{C} 22$ & $1.369(11)$ \\
\hline C22 & $\mathrm{C} 23$ & $1.392(11)$ \\
\hline C23 & $\mathrm{C} 24$ & $1.392(10)$ \\
\hline $\mathrm{C} 25$ & $\mathrm{C} 26$ & $1.389(10)$ \\
\hline $\mathrm{C} 25$ & C30 & $1.386(10)$ \\
\hline C26 & $\mathrm{C} 27$ & $1.372(11)$ \\
\hline $\mathrm{C} 27$ & C28 & $1.378(11)$ \\
\hline C28 & C29 & $1.385(11)$ \\
\hline C29 & C30 & $1.389(11)$ \\
\hline C31 & C32 & $1.383(9)$ \\
\hline C31 & C36 & $1.384(10)$ \\
\hline C32 & C33 & $1.384(10)$ \\
\hline C33 & C34 & $1.365(11)$ \\
\hline C34 & C35 & $1.364(11)$ \\
\hline C35 & C36 & $1.396(11)$ \\
\hline $\mathrm{Cl} 1$ & 013 & $1.437(6)$ \\
\hline $\mathrm{Cl} 1$ & 014 & $1.412(7)$ \\
\hline $\mathrm{Cl} 1$ & 015 & $1.412(7)$ \\
\hline $\mathrm{Cl} 1$ & 016 & $1.416(8)$ \\
\hline $\mathrm{Cl} 2$ & $017^{1}$ & $1.368(9)$ \\
\hline $\mathrm{Cl} 2$ & 017 & $1.369(9)$ \\
\hline $\mathrm{Cl} 2$ & 018 & $1.329(10)$ \\
\hline $\mathrm{Cl} 2$ & $018^{1}$ & $1.329(10)$ \\
\hline $\mathrm{Cl} 3$ & 019 & $1.370(9)$ \\
\hline $\mathrm{Cl} 3$ & 020 & $1.484(10)$ \\
\hline $\mathrm{Cl} 3$ & 021 & $1.467(11)$ \\
\hline $\mathrm{Cl} 3$ & 022 & $1.381(15)$ \\
\hline $\mathrm{Cl} 3 \mathrm{~A}$ & 019 & $1.573(12)$ \\
\hline $\mathrm{Cl} 3 \mathrm{~A}$ & 020 & $1.417(12)$ \\
\hline $\mathrm{Cl} 3 \mathrm{~A}$ & 021 & $1.233(12)$ \\
\hline $\mathrm{Cl} 3 \mathrm{~A}$ & O22A & $1.409(17)$ \\
\hline P3 & 03 & $1.500(5)$ \\
\hline P3 & C37 & $1.796(8)$ \\
\hline P3 & $\mathrm{C} 43$ & $1.783(8)$ \\
\hline P3 & C49 & $1.807(7)$ \\
\hline C37 & C38 & $1.379(11)$ \\
\hline C37 & $\mathrm{C} 42$ & $1.405(11)$ \\
\hline C38 & C39 & $1.394(12)$ \\
\hline C39 & $\mathrm{C} 40$ & $1.391(13)$ \\
\hline $\mathrm{C} 40$ & C41 & $1.354(13)$ \\
\hline C41 & C42 & $1.382(11)$ \\
\hline C43 & C44 & $1.370(11)$ \\
\hline
\end{tabular}

\begin{tabular}{|c|c|c|}
\hline Atom & Atom & Length/Å \\
\hline$\overline{\mathrm{C} 43}$ & C48 & $1.418(11)$ \\
\hline C44 & $\mathrm{C} 45$ & $1.399(12)$ \\
\hline C45 & $\mathrm{C} 46$ & $1.401(13)$ \\
\hline C46 & $\mathrm{C} 47$ & $1.362(13)$ \\
\hline $\mathrm{C} 47$ & $\mathrm{C} 48$ & $1.368(13)$ \\
\hline C49 & $\mathrm{C} 50$ & $1.402(12)$ \\
\hline C49 & C54 & $1.370(12)$ \\
\hline $\mathrm{C} 50$ & C51 & $1.412(11)$ \\
\hline C51 & C52 & $1.352(15)$ \\
\hline C52 & C53 & $1.369(15)$ \\
\hline C53 & C54 & $1.425(12)$ \\
\hline $\mathrm{P} 4$ & 04 & $1.498(5)$ \\
\hline $\mathrm{P} 4$ & C55 & $1.805(9)$ \\
\hline P4 & C61 & $1.802(10)$ \\
\hline P4 & C67 & $1.816(8)$ \\
\hline C55 & C56 & $1.413(13)$ \\
\hline C55 & $\mathrm{C} 60$ & $1.378(13)$ \\
\hline C56 & C57 & $1.393(15)$ \\
\hline C57 & C58 & $1.351(17)$ \\
\hline C58 & C59 & $1.370(16)$ \\
\hline C59 & $\mathrm{C} 60$ & $1.404(13)$ \\
\hline C61 & C62 & $1.382(11)$ \\
\hline C61 & C66 & $1.397(12)$ \\
\hline C62 & C63 & $1.383(14)$ \\
\hline C63 & C64 & $1.361(14)$ \\
\hline C64 & C65 & $1.369(13)$ \\
\hline C65 & C66 & $1.383(14)$ \\
\hline C67 & C68 & $1.391(14)$ \\
\hline C67 & $\mathrm{C} 72$ & $1.373(13)$ \\
\hline C68 & C69 & $1.406(13)$ \\
\hline C69 & C70 & $1.371(15)$ \\
\hline $\mathrm{C} 70$ & C71 & $1.348(16)$ \\
\hline C71 & C72 & $1.410(13)$ \\
\hline P5 & 05 & $1.496(5)$ \\
\hline P5 & C73 & $1.795(8)$ \\
\hline P5 & C79 & $1.790(8)$ \\
\hline P5 & C85 & $1.790(8)$ \\
\hline C73 & C74 & $1.394(11)$ \\
\hline C73 & C78 & $1.368(12)$ \\
\hline C74 & C75 & $1.386(13)$ \\
\hline C75 & C76 & $1.358(16)$ \\
\hline C76 & $\mathrm{C} 77$ & $1.331(16)$ \\
\hline C77 & C78 & $1.407(12)$ \\
\hline C79 & $\mathrm{C} 80$ & $1.389(11)$ \\
\hline C79 & C84 & $1.384(10)$ \\
\hline $\mathrm{C} 80$ & C81 & $1.384(12)$ \\
\hline C81 & $\mathrm{C} 82$ & $1.385(11)$ \\
\hline C82 & C83 & $1.383(11)$ \\
\hline C83 & C84 & $1.402(11)$ \\
\hline C85 & C86 & $1.381(11)$ \\
\hline C85 & C90 & $1.366(11)$ \\
\hline C86 & $\mathrm{C} 87$ & $1.390(12)$ \\
\hline C87 & C88 & $1.374(13)$ \\
\hline C88 & C89 & $1.367(13)$ \\
\hline C89 & C90 & $1.405(12)$ \\
\hline P6 & 06 & $1.508(6)$ \\
\hline P6 & C91 & $1.778(9)$ \\
\hline P6 & C97 & $1.798(9)$ \\
\hline P6 & C103 & $1.778(9)$ \\
\hline C91 & C92 & $1.393(13)$ \\
\hline C91 & C96 & $1.397(11)$ \\
\hline C92 & C93 & $1.377(13)$ \\
\hline
\end{tabular}




\begin{tabular}{lll}
\hline Atom & Atom & Length/A \\
\hline C93 & C94 & $1.373(13)$ \\
C94 & C95 & $1.378(14)$ \\
C95 & C96 & $1.396(12)$ \\
C97 & C98 & $1.396(13)$ \\
C97 & C102 & $1.408(14)$ \\
C98 & C99 & $1.399(14)$ \\
C99 & C100 & $1.370(17)$ \\
C100 & C101 & $1.369(16)$ \\
C101 & C102 & $1.398(13)$ \\
C103 & C104 & $1.390(12)$ \\
C103 & C108 & $1.385(12)$ \\
C104 & C105 & $1.378(14)$ \\
C105 & C106 & $1.378(14)$ \\
C106 & C107 & $1.353(14)$ \\
C107 & C108 & $1.420(13)$ \\
P7 & 07 & $1.495(6)$ \\
P7 & C109 & $1.782(10)$ \\
P7 & C115 & $1.766(12)$ \\
P7 & C121 & $1.803(13)$ \\
C109 & C110 & $1.359(13)$
\end{tabular}

\begin{tabular}{lll}
\hline Atom & Atom & Length/A \\
\hline $\mathrm{C} 109$ & $\mathrm{C} 114$ & $1.397(15)$ \\
$\mathrm{C} 110$ & $\mathrm{C} 111$ & $1.392(13)$ \\
$\mathrm{C} 111$ & $\mathrm{C} 112$ & $1.358(15)$ \\
$\mathrm{C} 112$ & $\mathrm{C} 113$ & $1.372(19)$ \\
$\mathrm{C} 113$ & $\mathrm{C} 114$ & $1.398(19)$ \\
$\mathrm{C} 115$ & $\mathrm{C} 116$ & $1.41(2)$ \\
$\mathrm{C} 115$ & $\mathrm{C} 120$ & $1.394(19)$ \\
$\mathrm{C} 116$ & $\mathrm{C} 117$ & $1.47(2)$ \\
$\mathrm{C} 117$ & $\mathrm{C} 118$ & $1.52(3)$ \\
$\mathrm{C} 118$ & $\mathrm{C} 119$ & $1.35(3)$ \\
$\mathrm{C} 119$ & $\mathrm{C} 120$ & $1.31(2)$ \\
$\mathrm{C} 121$ & $\mathrm{C} 122$ & $1.405(17)$ \\
$\mathrm{C} 121$ & $\mathrm{C} 126$ & $1.350(19)$ \\
$\mathrm{C} 122$ & $\mathrm{C} 123$ & $1.36(2)$ \\
$\mathrm{C} 123$ & $\mathrm{C} 124$ & $1.36(3)$ \\
$\mathrm{C} 124$ & $\mathrm{C} 125$ & $1.47(2)$ \\
$\mathrm{C} 125$ & $\mathrm{C} 126$ & $1.445(18)$ \\
---- & & \\
$11-\mathrm{x},+\mathrm{y}, 1 / 2-\mathrm{z}$ &
\end{tabular}

Table 3: Bond Angles in .

\begin{tabular}{|c|c|c|c|}
\hline Atom & Atom & Atom & Angle ${ }^{\circ}$ \\
\hline$\overline{01}$ & Er1 & 02 & $172.5(2)$ \\
\hline 01 & Er1 & 08 & $87.0(2)$ \\
\hline 01 & Er1 & 09 & 89.61(19) \\
\hline 01 & Er1 & 010 & $96.3(2)$ \\
\hline 01 & Er1 & 011 & $92.2(2)$ \\
\hline 01 & Er1 & 012 & $86.30(19)$ \\
\hline 02 & Er1 & 08 & 86.08(18) \\
\hline 02 & Er1 & 09 & $91.74(18)$ \\
\hline 02 & Er1 & 010 & $91.14(19)$ \\
\hline 02 & Er1 & 011 & $91.53(19)$ \\
\hline 02 & Er1 & 012 & $88.72(17)$ \\
\hline 08 & Er1 & 012 & $71.74(17)$ \\
\hline 09 & Er1 & 08 & $77.07(18)$ \\
\hline 09 & Er1 & 012 & $148.71(17)$ \\
\hline 010 & Er1 & 08 & $147.70(18)$ \\
\hline 010 & Er1 & 09 & $70.84(18)$ \\
\hline 010 & Er1 & 012 & $140.45(17)$ \\
\hline 011 & Er1 & 08 & $143.06(18)$ \\
\hline 011 & Er1 & 09 & $139.86(18)$ \\
\hline 011 & Er1 & 010 & $69.10(17)$ \\
\hline 011 & Er1 & 012 & $71.36(17)$ \\
\hline 01 & $\mathrm{P} 1$ & $\mathrm{C} 1$ & $111.1(4)$ \\
\hline 01 & $\mathrm{P} 1$ & $\mathrm{C} 7$ & $110.1(4)$ \\
\hline 01 & $\mathrm{P} 1$ & C13 & $109.5(3)$ \\
\hline $\mathrm{C} 1$ & $\mathrm{P} 1$ & $\mathrm{C} 7$ & $108.0(4)$ \\
\hline $\mathrm{C} 1$ & $\mathrm{P} 1$ & C13 & $108.3(4)$ \\
\hline C13 & P1 & $\mathrm{C} 7$ & $109.8(4)$ \\
\hline 02 & $\mathrm{P} 2$ & C19 & $110.0(3)$ \\
\hline 02 & $\mathrm{P} 2$ & $\mathrm{C} 25$ & $110.7(3)$ \\
\hline 02 & $\mathrm{P} 2$ & C31 & $109.2(3)$ \\
\hline $\mathrm{C} 25$ & $\mathrm{P} 2$ & C19 & $108.6(3)$ \\
\hline $\mathrm{C} 25$ & $\mathrm{P} 2$ & C31 & $110.6(3)$ \\
\hline C31 & $\mathrm{P} 2$ & C19 & $107.8(3)$ \\
\hline $\mathrm{P} 1$ & 01 & Er1 & $177.0(4)$ \\
\hline $\mathrm{P} 2$ & 02 & Er1 & $176.2(3)$ \\
\hline $\mathrm{C} 2$ & $\mathrm{C} 1$ & $\mathrm{P} 1$ & $120.1(7)$ \\
\hline
\end{tabular}

\begin{tabular}{|c|c|c|c|}
\hline Atom & Atom & Atom & Angle $/^{\circ}$ \\
\hline$\overline{\mathrm{C} 2}$ & C1 & C6 & $120.4(10)$ \\
\hline $\mathrm{C} 6$ & $\mathrm{C} 1$ & $\mathrm{P} 1$ & $119.4(8)$ \\
\hline $\mathrm{C} 3$ & $\mathrm{C} 2$ & $\mathrm{C} 1$ & $121.0(9)$ \\
\hline $\mathrm{C} 2$ & $\mathrm{C} 3$ & $\mathrm{C} 4$ & $118.8(11)$ \\
\hline $\mathrm{C} 5$ & $\mathrm{C} 4$ & C3 & $119.8(12)$ \\
\hline $\mathrm{C} 6$ & $\mathrm{C} 5$ & $\mathrm{C} 4$ & $121.0(12)$ \\
\hline $\mathrm{C} 5$ & C6 & C1 & $118.9(11)$ \\
\hline C8 & $\mathrm{C} 7$ & P1 & $121.9(6)$ \\
\hline C12 & $\mathrm{C} 7$ & P1 & $118.5(7)$ \\
\hline C12 & $\mathrm{C} 7$ & $\mathrm{C} 8$ & $119.5(8)$ \\
\hline $\mathrm{C} 9$ & $\mathrm{C} 8$ & $\mathrm{C} 7$ & $119.7(8)$ \\
\hline $\mathrm{C} 8$ & $\mathrm{C} 9$ & $\mathrm{C} 10$ & $119.0(9)$ \\
\hline C11 & C10 & C9 & $120.8(9)$ \\
\hline C12 & C11 & C10 & $119.5(9)$ \\
\hline C11 & C12 & $\mathrm{C} 7$ & $121.4(9)$ \\
\hline C14 & C13 & P1 & $122.4(8)$ \\
\hline C14 & C13 & C18 & $120.0(8)$ \\
\hline C18 & C13 & P1 & $117.5(6)$ \\
\hline C13 & C14 & C15 & $118.9(10)$ \\
\hline C16 & C15 & C14 & $120.6(10)$ \\
\hline C17 & C16 & C15 & $119.8(9)$ \\
\hline C16 & $\mathrm{C} 17$ & C18 & $119.7(10)$ \\
\hline C17 & C18 & C13 & $120.9(9)$ \\
\hline $\mathrm{C} 20$ & C19 & $\mathrm{P} 2$ & $117.8(5)$ \\
\hline $\mathrm{C} 20$ & C19 & $\mathrm{C} 24$ & $121.0(6)$ \\
\hline $\mathrm{C} 24$ & C19 & $\mathrm{P} 2$ & $121.2(5)$ \\
\hline $\mathrm{C} 21$ & $\mathrm{C} 20$ & C19 & $118.6(7)$ \\
\hline $\mathrm{C} 22$ & $\mathrm{C} 21$ & $\mathrm{C} 20$ & $121.2(8)$ \\
\hline $\mathrm{C} 21$ & $\mathrm{C} 22$ & $\mathrm{C} 23$ & $120.1(7)$ \\
\hline $\mathrm{C} 24$ & $\mathrm{C} 23$ & $\mathrm{C} 22$ & $120.2(7)$ \\
\hline $\mathrm{C} 23$ & $\mathrm{C} 24$ & C19 & $118.8(7)$ \\
\hline $\mathrm{C} 26$ & $\mathrm{C} 25$ & $\mathrm{P} 2$ & $117.5(5)$ \\
\hline C30 & $\mathrm{C} 25$ & $\mathrm{P} 2$ & $123.1(6)$ \\
\hline C30 & $\mathrm{C} 25$ & C26 & $119.4(7)$ \\
\hline $\mathrm{C} 27$ & $\mathrm{C} 26$ & $\mathrm{C} 25$ & $120.4(7)$ \\
\hline C26 & $\mathrm{C} 27$ & $\mathrm{C} 28$ & $120.8(8)$ \\
\hline
\end{tabular}




\begin{tabular}{|c|c|c|c|}
\hline Atom & Atom & Atom & Angle $/{ }^{\circ}$ \\
\hline$\overline{\mathrm{C} 27}$ & $\mathrm{C} 28$ & $\mathrm{C} 29$ & $119.1(8)$ \\
\hline $\mathrm{C} 28$ & $\mathrm{C} 29$ & $\mathrm{C} 30$ & $120.7(8)$ \\
\hline $\mathrm{C} 25$ & C30 & $\mathrm{C} 29$ & $119.6(7)$ \\
\hline C32 & C31 & $\mathrm{P} 2$ & $121.9(5)$ \\
\hline C32 & C31 & C36 & $120.6(7)$ \\
\hline C36 & C31 & $\mathrm{P} 2$ & $117.4(5)$ \\
\hline C31 & C32 & C33 & $119.3(7)$ \\
\hline C34 & C33 & C32 & $120.2(7)$ \\
\hline C35 & C34 & C33 & $120.9(8)$ \\
\hline C34 & C35 & C36 & $120.1(8)$ \\
\hline C31 & C36 & C35 & $118.9(7)$ \\
\hline 014 & $\mathrm{Cl} 1$ & 013 & $111.0(4)$ \\
\hline 014 & $\mathrm{Cl} 1$ & 015 & $110.7(5)$ \\
\hline 014 & $\mathrm{Cl} 1$ & 016 & $110.1(5)$ \\
\hline 015 & $\mathrm{Cl} 1$ & 013 & $108.9(4)$ \\
\hline 015 & $\mathrm{Cl} 1$ & 016 & $104.8(5)$ \\
\hline 016 & $\mathrm{Cl} 1$ & 013 & $111.2(5)$ \\
\hline $017^{1}$ & $\mathrm{Cl} 2$ & 017 & $115.5(10)$ \\
\hline $018^{1}$ & $\mathrm{Cl} 2$ & 017 & $105.5(7)$ \\
\hline 018 & $\mathrm{Cl} 2$ & 017 & $109.0(8)$ \\
\hline 018 & $\mathrm{Cl} 2$ & $017^{1}$ & $105.5(7)$ \\
\hline $018^{1}$ & $\mathrm{Cl} 2$ & $017^{1}$ & $109.0(8)$ \\
\hline 018 & $\mathrm{Cl} 2$ & $018^{1}$ & $112.7(16)$ \\
\hline 019 & $\mathrm{Cl} 3$ & 020 & $108.2(7)$ \\
\hline 019 & $\mathrm{Cl} 3$ & 021 & $107.6(7)$ \\
\hline 019 & $\mathrm{Cl} 3$ & 022 & $112.8(8)$ \\
\hline 021 & $\mathrm{Cl} 3$ & 020 & $105.3(8)$ \\
\hline 022 & $\mathrm{Cl} 3$ & 020 & $110.8(7)$ \\
\hline 022 & $\mathrm{Cl} 3$ & 021 & $111.8(7)$ \\
\hline 020 & $\mathrm{Cl} 3 \mathrm{~A}$ & 019 & $101.2(9)$ \\
\hline 021 & $\mathrm{Cl} 3 \mathrm{~A}$ & 019 & $108.8(9)$ \\
\hline 021 & $\mathrm{Cl} 3 \mathrm{~A}$ & 020 & $124.5(10)$ \\
\hline 021 & $\mathrm{Cl} 3 \mathrm{~A}$ & O22A & $121.8(16)$ \\
\hline 022A & $\mathrm{Cl} 3 \mathrm{~A}$ & 019 & $89.8(13)$ \\
\hline 022A & $\mathrm{Cl} 3 \mathrm{~A}$ & 020 & $103.5(14)$ \\
\hline 03 & P3 & C37 & $110.3(4)$ \\
\hline 03 & P3 & $\mathrm{C} 43$ & $113.7(3)$ \\
\hline 03 & P3 & C49 & $109.4(3)$ \\
\hline C37 & P3 & C49 & $107.3(3)$ \\
\hline C43 & P3 & C37 & $109.5(4)$ \\
\hline C43 & P3 & C49 & $106.4(4)$ \\
\hline C38 & C37 & P3 & $119.0(6)$ \\
\hline C38 & C37 & C42 & $119.8(8)$ \\
\hline C42 & C37 & P3 & $121.3(6)$ \\
\hline C37 & C38 & C39 & $120.4(8)$ \\
\hline $\mathrm{C} 40$ & C39 & C38 & $119.3(9)$ \\
\hline C41 & $\mathrm{C} 40$ & C39 & $120.0(9)$ \\
\hline $\mathrm{C} 40$ & $\mathrm{C} 41$ & C42 & $121.9(9)$ \\
\hline C41 & $\mathrm{C} 42$ & C37 & $118.6(8)$ \\
\hline C44 & $\mathrm{C} 43$ & P3 & $125.2(6)$ \\
\hline C44 & $\mathrm{C} 43$ & $\mathrm{C} 48$ & $118.5(8)$ \\
\hline C48 & $\mathrm{C} 43$ & P3 & $116.2(6)$ \\
\hline C43 & $\mathrm{C} 44$ & $\mathrm{C} 45$ & $120.7(8)$ \\
\hline C44 & $\mathrm{C} 45$ & $\mathrm{C} 46$ & $119.2(9)$ \\
\hline C47 & $\mathrm{C} 46$ & $\mathrm{C} 45$ & $120.7(9)$ \\
\hline C46 & $\mathrm{C} 47$ & $\mathrm{C} 48$ & $120.0(9)$ \\
\hline $\mathrm{C} 47$ & $\mathrm{C} 48$ & $\mathrm{C} 43$ & 121.1(8) \\
\hline C50 & C49 & P3 & $121.2(6)$ \\
\hline C54 & $\mathrm{C} 49$ & P3 & $117.7(7)$ \\
\hline C54 & $\mathrm{C} 49$ & $\mathrm{C} 50$ & $121.1(8)$ \\
\hline C49 & C50 & C51 & $117.8(9)$ \\
\hline C52 & C51 & C50 & $120.6(10)$ \\
\hline
\end{tabular}

\begin{tabular}{|c|c|c|c|}
\hline Atom & Atom & Atom & Angle $/^{\circ}$ \\
\hline$\overline{\mathrm{C} 51}$ & C52 & C53 & $122.4(9)$ \\
\hline C52 & C53 & C54 & $118.2(10)$ \\
\hline C49 & $\mathrm{C} 54$ & C53 & $119.9(10)$ \\
\hline 04 & $\mathrm{P} 4$ & C55 & $111.8(4)$ \\
\hline 04 & $\mathrm{P} 4$ & C61 & $110.6(4)$ \\
\hline 04 & $\mathrm{P} 4$ & C67 & $112.7(3)$ \\
\hline C55 & $\mathrm{P} 4$ & C67 & $106.1(4)$ \\
\hline C61 & $\mathrm{P} 4$ & C55 & $108.0(4)$ \\
\hline C61 & $\mathrm{P} 4$ & C67 & $107.4(4)$ \\
\hline C56 & C55 & $\mathrm{P} 4$ & $117.0(8)$ \\
\hline C60 & C55 & $\mathrm{P} 4$ & $122.5(7)$ \\
\hline C60 & C55 & C56 & $120.5(9)$ \\
\hline C57 & C56 & C55 & $117.7(11)$ \\
\hline C58 & C57 & C56 & $120.8(12)$ \\
\hline C57 & C58 & C59 & $122.5(11)$ \\
\hline C58 & C59 & $\mathrm{C} 60$ & $118.1(12)$ \\
\hline C55 & C60 & C59 & $120.3(10)$ \\
\hline C62 & C61 & $\mathrm{P} 4$ & $121.5(8)$ \\
\hline C62 & C61 & C66 & $119.7(10)$ \\
\hline C66 & C61 & $\mathrm{P} 4$ & $118.8(6)$ \\
\hline C61 & C62 & C63 & $119.8(9)$ \\
\hline C64 & C63 & C62 & $120.1(9)$ \\
\hline C63 & C64 & C65 & $121.1(11)$ \\
\hline C64 & C65 & C66 & $119.9(10)$ \\
\hline C65 & C66 & C61 & $119.5(8)$ \\
\hline C68 & C67 & $\mathrm{P} 4$ & $115.8(7)$ \\
\hline C72 & C67 & $\mathrm{P} 4$ & $122.7(8)$ \\
\hline C72 & C67 & C68 & $121.5(9)$ \\
\hline C67 & C68 & C69 & $118.3(10)$ \\
\hline C70 & C69 & C68 & $119.7(12)$ \\
\hline C71 & C70 & C69 & $121.5(10)$ \\
\hline C70 & C71 & C72 & $120.3(11)$ \\
\hline C67 & C72 & C71 & $118.5(11)$ \\
\hline 05 & P5 & C73 & $111.8(4)$ \\
\hline 05 & P5 & C79 & $110.4(3)$ \\
\hline 05 & P5 & $\mathrm{C} 85$ & $111.4(3)$ \\
\hline C79 & P5 & C73 & $107.8(4)$ \\
\hline C79 & P5 & C85 & $109.0(4)$ \\
\hline C85 & P5 & C73 & $106.4(4)$ \\
\hline C74 & C73 & P5 & $119.6(7)$ \\
\hline C78 & C73 & P5 & $122.0(7)$ \\
\hline C78 & C73 & C74 & $118.4(8)$ \\
\hline $\mathrm{C} 75$ & C74 & C73 & $120.4(10)$ \\
\hline C76 & C75 & C74 & $120.5(11)$ \\
\hline C77 & C76 & C75 & $119.5(11)$ \\
\hline C76 & C77 & C78 & $122.0(12)$ \\
\hline C73 & C78 & C77 & $119.2(10)$ \\
\hline C80 & C79 & P5 & $118.7(6)$ \\
\hline C84 & C79 & P5 & $122.1(6)$ \\
\hline C84 & C79 & $\mathrm{C} 80$ & $119.2(7)$ \\
\hline C81 & $\mathrm{C} 80$ & C79 & $120.8(8)$ \\
\hline C80 & C81 & C82 & $119.7(8)$ \\
\hline C83 & C82 & C81 & $120.4(8)$ \\
\hline C82 & C83 & C84 & $119.5(8)$ \\
\hline C79 & $\mathrm{C} 84$ & C83 & $120.4(7)$ \\
\hline C86 & $\mathrm{C} 85$ & P5 & $123.8(6)$ \\
\hline C90 & C85 & P5 & $117.1(6)$ \\
\hline C90 & C85 & C86 & $119.1(8)$ \\
\hline C85 & C86 & C87 & 118.7(9) \\
\hline C88 & C87 & C86 & $121.8(8)$ \\
\hline C89 & C88 & C87 & $120.0(9)$ \\
\hline C88 & C89 & C90 & $118.0(9)$ \\
\hline
\end{tabular}




\begin{tabular}{|c|c|c|c|}
\hline Atom & Atom & Atom & Angle $/^{\circ}$ \\
\hline$\overline{\mathrm{C} 85}$ & C90 & C89 & $122.4(8)$ \\
\hline 06 & P6 & C91 & $109.7(4)$ \\
\hline 06 & P6 & C97 & $113.0(4)$ \\
\hline 06 & P6 & C103 & $109.2(4)$ \\
\hline C91 & P6 & C97 & $106.0(4)$ \\
\hline C91 & P6 & C103 & 111.1(4) \\
\hline C103 & P6 & C97 & $107.9(5)$ \\
\hline C92 & C91 & P6 & $120.0(6)$ \\
\hline C92 & C91 & C96 & $117.6(8)$ \\
\hline C96 & C91 & P6 & 122.4(7) \\
\hline C93 & C92 & C91 & $121.9(9)$ \\
\hline C94 & C93 & C92 & $120.0(10)$ \\
\hline C93 & C94 & C95 & $119.7(9)$ \\
\hline C94 & C95 & C96 & $120.6(9)$ \\
\hline C95 & C96 & C91 & $120.2(9)$ \\
\hline C98 & C97 & P6 & $118.6(8)$ \\
\hline C98 & C97 & C102 & $121.0(9)$ \\
\hline C102 & C97 & P6 & $120.3(7)$ \\
\hline C97 & C98 & C99 & 118.3(12) \\
\hline C100 & C99 & C98 & $121.3(12)$ \\
\hline C101 & C100 & C99 & $119.9(11)$ \\
\hline C100 & C101 & C102 & $121.7(13)$ \\
\hline C101 & C102 & C97 & $117.7(11)$ \\
\hline C104 & C103 & P6 & 124.4(7) \\
\hline C108 & C103 & P6 & $117.3(7)$ \\
\hline C108 & C103 & C104 & 118.1(9) \\
\hline C105 & C104 & C103 & $120.9(10)$ \\
\hline C104 & C105 & C106 & $121.0(10)$ \\
\hline C107 & C106 & C105 & $119.5(10)$ \\
\hline C106 & C107 & C108 & $120.3(9)$ \\
\hline C103 & C108 & C107 & $120.2(9)$ \\
\hline 07 & P7 & C109 & $111.4(4)$ \\
\hline 07 & P7 & C115 & $114.0(4)$ \\
\hline 07 & P7 & C121 & $110.4(6)$ \\
\hline C109 & P7 & C121 & $107.2(5)$ \\
\hline C115 & P7 & C109 & $108.5(6)$ \\
\hline C115 & P7 & C121 & $105.0(7)$ \\
\hline C110 & C109 & P7 & $117.4(8)$ \\
\hline C110 & C109 & C114 & $119.6(10)$ \\
\hline C114 & C109 & P7 & $123.1(9)$ \\
\hline C109 & C110 & C111 & 119.6(10) \\
\hline C112 & C111 & C110 & $120.9(11)$ \\
\hline C111 & C112 & C113 & $120.8(12)$ \\
\hline C112 & C113 & C114 & 118.5(14) \\
\hline C109 & C114 & C113 & $120.5(13)$ \\
\hline C116 & C115 & P7 & $118.9(12)$ \\
\hline C120 & C115 & P7 & $120.2(14)$ \\
\hline C120 & C115 & C116 & $120.0(14)$ \\
\hline C115 & C116 & C117 & $118.0(19)$ \\
\hline C116 & C117 & C118 & 110.8(19) \\
\hline C119 & C118 & C117 & 129.3(17) \\
\hline C120 & C119 & C118 & $112(2)$ \\
\hline C119 & C120 & C115 & $128(2)$ \\
\hline C122 & C121 & P7 & $119.3(14)$ \\
\hline C126 & C121 & P7 & 119.3(9) \\
\hline C126 & C121 & C122 & $121.3(14)$ \\
\hline C123 & C122 & C121 & $122(2)$ \\
\hline C124 & C123 & C122 & $118(2)$ \\
\hline C123 & C124 & C125 & $124.2(18)$ \\
\hline C126 & C125 & C124 & 113.5(18) \\
\hline C121 & C126 & C125 & $121.2(14)$ \\
\hline
\end{tabular}




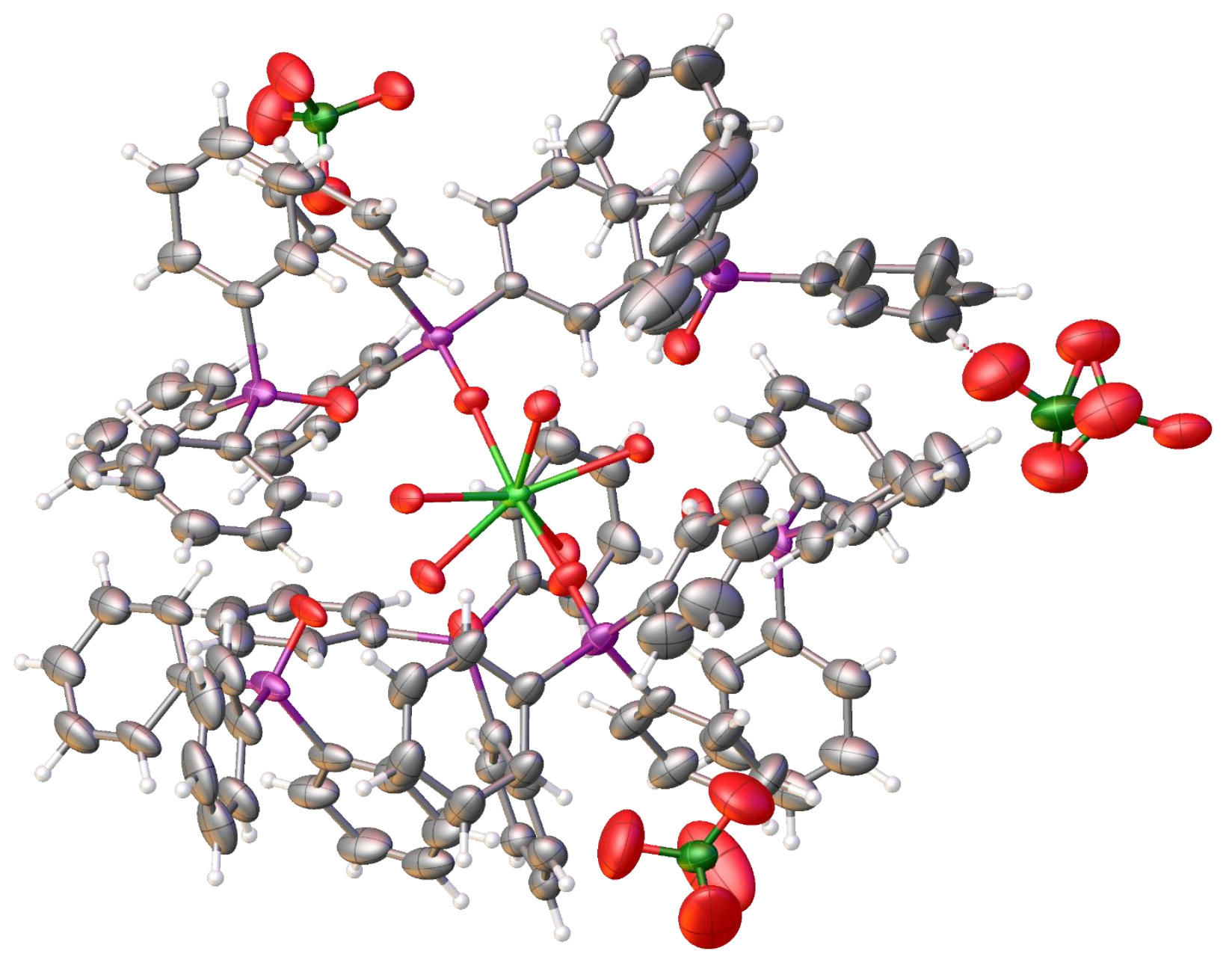




\section{Crystal Data and Experimental $\left[\mathrm{Nd}\left(\mathrm{k}^{3}-\mathrm{O}, \mathrm{O}, \mathrm{O}^{\prime}-\mathrm{S}_{3} \mathrm{O}_{6}\right)\left(\mathrm{Ph}_{3} \mathrm{PO}\right)_{4}\left(\mathrm{H}_{2} \mathrm{O}\right)\right] \cdot 3\left[\mathrm{ClO}_{4}\right]$}

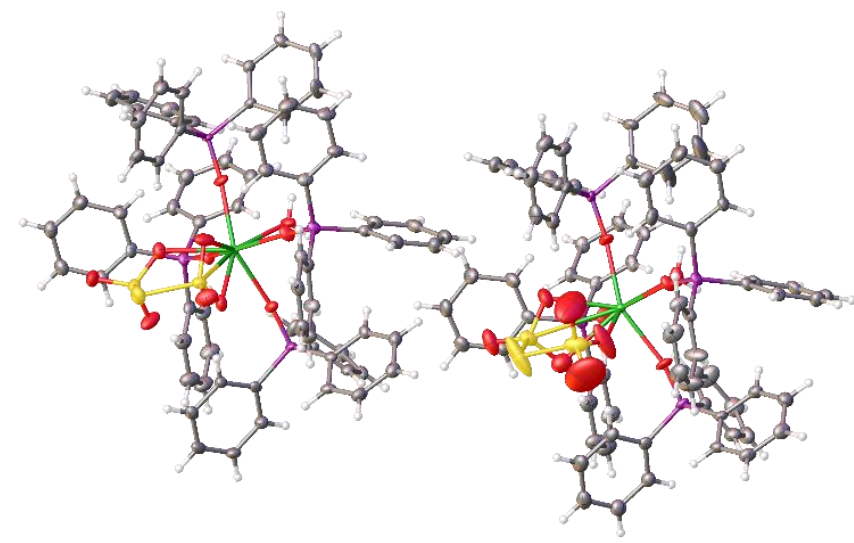

Figure 1: Thermal ellipsoids drawn at the $50 \%$ probability level.

Experimental. A suitable colourless block-shaped crystal $\left(0.300 \times 0.250 \times 0.200 \mathrm{~mm}^{3}\right)$ was selected and mounted on a MITIGEN holder in perfluoroether oil on a Rigaku FRE+ diffractometer equipped with VHF Varimax confocal mirrors and an AFC12 goniometer and HyPix 6000HE detector. The crystal was kept at a steady $T=100(2) \mathrm{K}$ during data collection. The structure was solved with the ShelXT (Sheldrick, 2015) structure solution program using the Intrinsic Phasing solution method and by using Olex2 (Dolomanov et al., 2009) as the graphical interface. The model was refined with version 2014/7 of ShelXL (Sheldrick, 2015) using Least Squares minimisation.

Crystal Data. $\mathrm{C}_{72} \mathrm{H}_{62} \mathrm{NdO}_{11} \mathrm{P}_{4} \mathrm{~S}_{3}, M_{r}=1467.51$, tetragonal, $P 4_{3}$ (No. 78), $\mathrm{a}=19.8658(2) \AA, \mathrm{b}=19.8658(2) \AA, \mathrm{c}=$ 34.7894(6) $\AA, \alpha=\beta=\gamma=90^{\circ}, V=13729.6(4) \AA^{3}, T=$ $100(2) \mathrm{K}, Z=8, Z^{\prime}=2, \mu\left(\mathrm{MoK}_{\alpha}\right)=1.000 \mathrm{~mm}^{-1}, 145177$ reflections measured, 31470 unique $\left(R_{\text {int }}=0.0395\right)$ which were used in all calculations. The final $w R_{2}$ was 0.1438 (all data) and $R_{1}$ was 0.0550 (I > 2(I)).

\begin{tabular}{|c|c|}
\hline Formula & $\mathrm{C}_{72} \mathrm{H}_{62} \mathrm{NdO}_{11} \mathrm{P}_{4} \mathrm{~S}_{3}$ \\
\hline$D_{\text {calc. }} / \mathrm{g} \mathrm{cm}^{-3}$ & 1.420 \\
\hline$\mu / \mathrm{mm}^{-1}$ & 1.000 \\
\hline Formula Weight & 1467.51 \\
\hline Colour & colourless \\
\hline Shape & block \\
\hline Size $/ \mathrm{mm}^{3}$ & $0.300 \times 0.250 \times 0.200$ \\
\hline$T / \mathrm{K}$ & $100(2)$ \\
\hline Crystal System & tetragonal \\
\hline Flack Parameter & $0.338(12)$ \\
\hline Hooft Parameter & $0.362(2)$ \\
\hline Space Group & $P 4_{3}$ \\
\hline$a / \AA ̊$ & $19.8658(2)$ \\
\hline$b / \AA$ & $19.8658(2)$ \\
\hline$c / \AA ̊$ & $34.7894(6)$ \\
\hline$\alpha /^{\circ}$ & 90 \\
\hline$\beta /^{\circ}$ & 90 \\
\hline$\gamma /^{\circ}$ & 90 \\
\hline $\mathrm{V} / \AA^{3}$ & $13729.6(4)$ \\
\hline$Z$ & 8 \\
\hline$Z^{\prime}$ & 2 \\
\hline Wavelength/A & 0.71075 \\
\hline Radiation type & $\mathrm{MoK}_{\alpha}$ \\
\hline$\Theta_{\min } /^{\circ}$ & 1.556 \\
\hline$\Theta_{\max } /^{\circ}$ & 27.483 \\
\hline Measured Refl. & 145177 \\
\hline Independent Refl. & 31470 \\
\hline $\begin{array}{l}\text { Reflections with I > } \\
2(\mathrm{I})\end{array}$ & 28997 \\
\hline$R_{\text {int }}$ & 0.0395 \\
\hline Parameters & 1632 \\
\hline Restraints & 1375 \\
\hline Largest Peak & 3.812 \\
\hline Deepest Hole & -1.784 \\
\hline GooF & 1.038 \\
\hline$w R_{2}$ (all data) & 0.1438 \\
\hline$w R_{2}$ & 0.1414 \\
\hline$R_{1}$ (all data) & 0.0590 \\
\hline$R_{1}$ & 0.0550 \\
\hline
\end{tabular}


Structure Quality Indicators

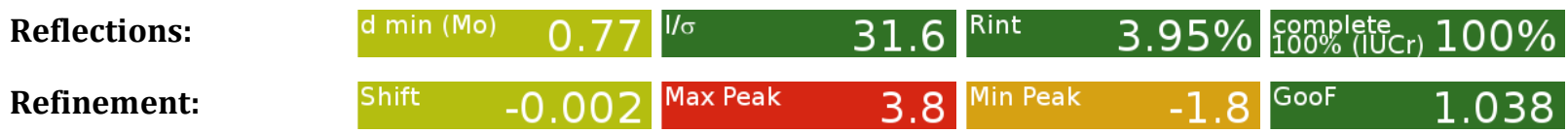

A colourless block-shaped crystal with dimensions $0.300 \times 0.250 \times 0.200 \mathrm{~mm}^{3}$ was mounted on a MITIGEN holder in perfluoroether oil. Data were collected using a Rigaku FRE+ diffractometer equipped with VHF Varimax confocal mirrors and an AFC12 goniometer and HyPix 6000HE detector, and equipped with an Oxford Cryosystems low-temperature device operating at $T=100(2) \mathrm{K}$.

Data were measured using $\omega$ scans of $0.5^{\circ}$ per frame for $0.5 \mathrm{~s}$ using $\mathrm{MoK}_{\alpha}$ radiation. The total number of runs and images was based on the strategy calculation from the program CrysAlisPro (Rigaku, V1.171.40.39a, 2019). The maximum resolution that was achieved was $\Theta=27.483^{\circ}(0.77 \AA$ ) .

The diffraction pattern was indexed and the unit cell was refined using CrysAlisPro (Rigaku, V1.171.40.39a, 2019) on 49914 reflections, $34 \%$ of the observed reflections.

Data reduction, scaling and absorption corrections were performed using CrysAlisPro (Rigaku, V1.171.40.39a, 2019). The final completeness is $99.90 \%$ out to $27.483^{\circ}$ in $\Theta$.

A multi-scan absorption correction was performed using CrysAlisPro 1.171.40.39a (Rigaku Oxford Diffraction, 2019) using spherical harmonics as implemented in SCALE3 ABSPACK. The absorption coefficient $\mu$ of this material is $1.000 \mathrm{~mm}^{-1}$ at this wavelength $(\lambda=0.71075 \AA)$ and the minimum and maximum transmissions are 0.815 and 1.000 .

The structure was solved and the space group $\mathrm{P4}_{3}$ (\# 78) determined by the ShelXT (Sheldrick, 2015) structure solution program using Intrinsic Phasing and refined by Least Squares using version 2014/7 of ShelXL (Sheldrick, 2015). All non-hydrogen atoms were refined anisotropically, apart from the two Nd atoms, which were refined isotropically. Hydrogen atom positions were calculated geometrically and refined using the riding model. There are some cavities available for solvents (532 and $600 \AA^{3}$ ). No solvents were identified and the structure was SQUEEZEd.

_refine_special_details: Refined as a 2-component inversion twin.

The value of $Z^{\prime}$ is 2 . This means that there are two independent molecules in the asymmetric unit.

The Flack parameter was refined to 0.338(12). Determination of absolute structure using Bayesian statistics on Bijvoet differences using the Olex2 results in 0.362(2). Note: The Flack parameter is used to determine chirality of the crystal studied, the value should be near 0 , a value of 1 means that the stereochemistry is wrong and the model should be inverted. A value of 0.5 means that the crystal consists of a racemic mixture of the two enantiomers. 
Reflection Statistics

$\begin{array}{llll}\begin{array}{l}\text { Total reflections (after } \\ \text { filtering) }\end{array} & 145281 & \text { Unique reflections } & 31470 \\ \text { Completeness } & 0.999 & \text { Mean I/ } \sigma & 19.46 \\ \text { hklmax collected } & (24,25,45) & \text { hklmin collected } & (-25,-19,-45) \\ \text { hklmax used } & (18,25,45) & \text { hklmin } \text { used } & (-17,0,-45) \\ \text { Lim } \mathrm{d}_{\max } \text { collected } & 100.0 & \text { Lim } \mathrm{d}_{\min } \text { collected } & 0.36 \\ \mathrm{~d}_{\max } \text { used } & 13.09 & \mathrm{~d}_{\min } \text { used } & 0.77 \\ \text { Friedel pairs } & 20730 & \text { Friedel pairs merged } & 0 \\ \text { Inconsistent equivalents } & 83 & \text { Rint } & 0.0395 \\ \text { Rsigma } & 0.0316 & \text { Intensity transformed } & 0 \\ \text { Omitted reflections } & 0 & \text { Omitted by user (OMIT hkl) } & 11 \\ \text { Multiplicity } & (38200,32603,9231,2152, & \text { Maximum multiplicity } & 17 \\ & 651,194,165) & & \\ \text { Removed systematic absences } & 93 & \text { Filtered off (Shel/OMIT) } & 0\end{array}$


Table 1: Bond Lengths in $\AA$

\begin{tabular}{|c|c|c|c|c|c|}
\hline Atom & Atom & Length/Å & Atom & Atom & Length/Å \\
\hline$\overline{\mathrm{Nd} 1}$ & $\mathrm{~S} 1$ & $3.2607(18)$ & $\overline{\mathrm{C} 27}$ & $\mathrm{C} 28$ & $1.393(10)$ \\
\hline Nd1 & 01 & $2.534(5)$ & $\mathrm{C} 28$ & C29 & $1.368(11)$ \\
\hline Nd1 & 02 & $2.840(7)$ & $\mathrm{C} 29$ & C30 & $1.379(11)$ \\
\hline $\mathrm{Nd} 1$ & 06 & $2.499(5)$ & $\mathrm{C} 31$ & C32 & $1.401(10)$ \\
\hline Nd1 & 07 & $2.360(5)$ & $\mathrm{C} 31$ & C36 & $1.381(10)$ \\
\hline $\mathrm{Nd} 1$ & 08 & $2.353(5)$ & $\mathrm{C} 32$ & C33 & $1.380(11)$ \\
\hline $\mathrm{Nd} 1$ & 09 & $2.365(5)$ & C33 & C34 & $1.401(12)$ \\
\hline Nd1 & 010 & $2.371(5)$ & $\mathrm{C} 34$ & C35 & $1.390(11)$ \\
\hline Nd1 & 011 & $2.478(6)$ & $\mathrm{C} 35$ & C36 & $1.393(10)$ \\
\hline S1 & $\mathrm{S} 2$ & $2.102(3)$ & $\mathrm{C} 37$ & C38 & $1.394(11)$ \\
\hline S1 & 01 & $1.475(5)$ & $\mathrm{C} 37$ & $\mathrm{C} 42$ & $1.385(10)$ \\
\hline S1 & 02 & $1.452(6)$ & C38 & C39 & $1.387(10)$ \\
\hline S1 & 03 & $1.414(6)$ & C39 & $\mathrm{C} 40$ & $1.363(13)$ \\
\hline S2 & S3 & $2.144(3)$ & $\mathrm{C} 40$ & C41 & $1.367(13)$ \\
\hline S3 & 04 & $1.411(6)$ & $\mathrm{C} 41$ & $\mathrm{C} 42$ & $1.391(10)$ \\
\hline S3 & 05 & $1.440(6)$ & $\mathrm{C} 43$ & C44 & $1.386(10)$ \\
\hline S3 & 06 & $1.442(5)$ & $\mathrm{C} 43$ & $\mathrm{C} 48$ & $1.416(10)$ \\
\hline P1 & 07 & $1.498(5)$ & $\mathrm{C} 44$ & $\mathrm{C} 45$ & $1.396(11)$ \\
\hline P1 & $\mathrm{C} 1$ & $1.797(7)$ & $\mathrm{C} 45$ & C46 & $1.391(12)$ \\
\hline $\mathrm{P} 1$ & $\mathrm{C} 7$ & $1.786(7)$ & $\mathrm{C} 46$ & $\mathrm{C} 47$ & $1.411(12)$ \\
\hline $\mathrm{P} 1$ & $\mathrm{C} 13$ & $1.797(8)$ & $\mathrm{C} 47$ & C48 & $1.377(11)$ \\
\hline P2 & 08 & $1.502(5)$ & $\mathrm{C} 49$ & C50 & $1.406(10)$ \\
\hline P2 & C19 & $1.791(7)$ & $\mathrm{C} 49$ & C54 & $1.370(9)$ \\
\hline P2 & $\mathrm{C} 25$ & $1.814(7)$ & $\mathrm{C} 50$ & C51 & $1.388(10)$ \\
\hline P2 & C31 & $1.789(7)$ & C51 & C52 & $1.379(11)$ \\
\hline P3 & 09 & $1.502(5)$ & C52 & C53 & $1.396(11)$ \\
\hline P3 & C37 & $1.795(7)$ & C53 & C54 & $1.396(10)$ \\
\hline P3 & $\mathrm{C} 43$ & $1.789(8)$ & C55 & C56 & $1.399(9)$ \\
\hline P3 & $\mathrm{C} 49$ & $1.798(7)$ & C55 & C60 & $1.402(9)$ \\
\hline $\mathrm{P} 4$ & 010 & $1.498(5)$ & C56 & C57 & $1.384(10)$ \\
\hline P4 & C55 & $1.794(7)$ & $\mathrm{C} 57$ & C58 & $1.385(11)$ \\
\hline P4 & C61 & $1.797(7)$ & C58 & C59 & $1.385(11)$ \\
\hline P4 & C67 & $1.803(7)$ & C59 & C60 & $1.386(10)$ \\
\hline $\mathrm{C} 1$ & $\mathrm{C} 2$ & $1.385(11)$ & C61 & C62 & $1.396(10)$ \\
\hline $\mathrm{C} 1$ & $\mathrm{C} 6$ & $1.391(10)$ & C61 & C66 & $1.388(10)$ \\
\hline $\mathrm{C} 2$ & $\mathrm{C} 3$ & $1.392(11)$ & $\mathrm{C} 62$ & C63 & $1.385(10)$ \\
\hline C3 & $\mathrm{C} 4$ & $1.376(12)$ & C63 & C64 & $1.380(11)$ \\
\hline $\mathrm{C} 4$ & $\mathrm{C} 5$ & $1.343(13)$ & $\mathrm{C} 64$ & C65 & $1.372(11)$ \\
\hline $\mathrm{C} 5$ & C6 & $1.407(10)$ & $\mathrm{C} 65$ & C66 & $1.397(10)$ \\
\hline C7 & $\mathrm{C} 8$ & $1.369(10)$ & $\mathrm{C} 67$ & C68 & $1.397(9)$ \\
\hline C7 & $\mathrm{C} 12$ & $1.410(10)$ & $\mathrm{C} 67$ & C72 & $1.375(10)$ \\
\hline $\mathrm{C} 8$ & C9 & $1.391(10)$ & C68 & C69 & $1.387(9)$ \\
\hline $\mathrm{C} 9$ & $\mathrm{C} 10$ & $1.396(11)$ & C69 & $\mathrm{C} 70$ & $1.390(11)$ \\
\hline C10 & $\mathrm{C} 11$ & $1.379(11)$ & $\mathrm{C} 70$ & C71 & $1.368(11)$ \\
\hline C11 & $\mathrm{C} 12$ & $1.372(11)$ & C71 & C72 & $1.405(10)$ \\
\hline C13 & $\mathrm{C} 14$ & $1.409(10)$ & $\mathrm{Nd} 2$ & S6 & $3.243(2)$ \\
\hline C13 & C18 & $1.386(10)$ & $\mathrm{Nd} 2$ & 012 & $2.431(7)$ \\
\hline C14 & C15 & $1.397(11)$ & $\mathrm{Nd} 2$ & 016 & $2.538(6)$ \\
\hline C15 & C16 & $1.390(12)$ & $\mathrm{Nd} 2$ & 017 & $2.692(7)$ \\
\hline C16 & $\mathrm{C} 17$ & $1.373(11)$ & $\mathrm{Nd} 2$ & 018 & $2.358(5)$ \\
\hline C17 & C18 & $1.393(11)$ & $\mathrm{Nd} 2$ & 019 & $2.332(5)$ \\
\hline C19 & $\mathrm{C} 20$ & $1.395(10)$ & $\mathrm{Nd} 2$ & 020 & $2.368(5)$ \\
\hline C19 & $\mathrm{C} 24$ & $1.406(10)$ & $\mathrm{Nd} 2$ & 021 & $2.360(5)$ \\
\hline $\mathrm{C} 20$ & $\mathrm{C} 21$ & $1.383(9)$ & $\mathrm{Nd} 2$ & 022 & $2.542(6)$ \\
\hline C21 & $\mathrm{C} 22$ & $1.410(13)$ & $\mathrm{S} 4$ & S5 & $2.151(4)$ \\
\hline $\mathrm{C} 22$ & $\mathrm{C} 23$ & $1.379(12)$ & $\mathrm{S} 4$ & 012 & $1.415(8)$ \\
\hline C23 & $\mathrm{C} 24$ & $1.388(10)$ & $\mathrm{S} 4$ & 013 & $1.382(11)$ \\
\hline $\mathrm{C} 25$ & $\mathrm{C} 26$ & $1.384(9)$ & $\mathrm{S} 4$ & 014 & $1.373(12)$ \\
\hline C25 & $\mathrm{C} 30$ & $1.386(10)$ & S5 & S6 & $2.076(4)$ \\
\hline $\mathrm{C} 26$ & $\mathrm{C} 27$ & $1.383(10)$ & S6 & 015 & $1.402(7)$ \\
\hline
\end{tabular}




\begin{tabular}{|c|c|c|}
\hline Atom & Atom & Length/Å \\
\hline$\overline{\mathrm{S} 6}$ & 016 & $1.453(6)$ \\
\hline S6 & 017 & $1.484(7)$ \\
\hline P5 & 018 & $1.500(5)$ \\
\hline P5 & C73 & $1.786(7)$ \\
\hline P5 & C79 & $1.793(7)$ \\
\hline P5 & C85 & $1.803(7)$ \\
\hline P6 & 019 & $1.514(5)$ \\
\hline P6 & C91 & $1.795(8)$ \\
\hline P6 & C97 & $1.790(7)$ \\
\hline P6 & C103 & $1.797(8)$ \\
\hline P7 & 020 & $1.502(5)$ \\
\hline P7 & C109 & $1.809(8)$ \\
\hline P7 & C115 & $1.779(7)$ \\
\hline P7 & C121 & $1.784(7)$ \\
\hline P8 & 021 & $1.495(5)$ \\
\hline P8 & C127 & $1.800(7)$ \\
\hline P8 & C133 & $1.797(7)$ \\
\hline P8 & C139 & $1.795(8)$ \\
\hline C73 & C74 & $1.405(11)$ \\
\hline C73 & $\mathrm{C} 78$ & $1.394(10)$ \\
\hline C74 & C75 & $1.391(11)$ \\
\hline C75 & C76 & $1.373(13)$ \\
\hline C76 & C77 & $1.387(12)$ \\
\hline C77 & C78 & $1.387(11)$ \\
\hline C79 & $\mathrm{C} 80$ & $1.381(10)$ \\
\hline C79 & C84 & $1.385(10)$ \\
\hline C80 & C81 & $1.394(11)$ \\
\hline C81 & C82 & $1.354(12)$ \\
\hline C82 & C83 & $1.389(11)$ \\
\hline C83 & C84 & $1.386(11)$ \\
\hline C85 & C86 & $1.397(9)$ \\
\hline C85 & C90 & $1.379(10)$ \\
\hline C86 & C87 & $1.401(10)$ \\
\hline C87 & C88 & $1.406(10)$ \\
\hline C88 & C89 & $1.402(11)$ \\
\hline C89 & C90 & $1.395(10)$ \\
\hline C91 & C92 & $1.387(10)$ \\
\hline C91 & C96 & $1.400(11)$ \\
\hline C92 & C93 & $1.393(10)$ \\
\hline C93 & C94 & $1.372(12)$ \\
\hline C94 & C95 & $1.380(12)$ \\
\hline C95 & C96 & $1.390(11)$ \\
\hline C97 & C98 & $1.373(10)$ \\
\hline C97 & C102 & $1.381(12)$ \\
\hline C98 & C99 & $1.384(11)$ \\
\hline
\end{tabular}

\begin{tabular}{|c|c|c|}
\hline Atom & Atom & Length/Å \\
\hline$\overline{\text { C99 }}$ & C100 & $1.382(16)$ \\
\hline C100 & C101 & $1.377(18)$ \\
\hline C101 & C102 & $1.351(15)$ \\
\hline C103 & C104 & $1.399(10)$ \\
\hline C103 & C108 & $1.389(11)$ \\
\hline C104 & C105 & $1.398(11)$ \\
\hline C105 & C106 & $1.385(12)$ \\
\hline C106 & C107 & $1.359(13)$ \\
\hline C107 & C108 & $1.383(12)$ \\
\hline C109 & C110 & $1.399(10)$ \\
\hline C109 & C114 & $1.389(9)$ \\
\hline C110 & C111 & $1.376(11)$ \\
\hline C111 & C112 & $1.412(12)$ \\
\hline C112 & C113 & $1.383(11)$ \\
\hline C113 & C114 & $1.391(10)$ \\
\hline C115 & C116 & $1.399(10)$ \\
\hline C115 & C120 & $1.417(9)$ \\
\hline C116 & C117 & $1.392(10)$ \\
\hline C117 & C118 & $1.395(11)$ \\
\hline C118 & C119 & $1.398(11)$ \\
\hline C119 & $\mathrm{C} 120$ & $1.369(10)$ \\
\hline C121 & C122 & $1.383(10)$ \\
\hline C121 & C126 & $1.387(10)$ \\
\hline C122 & C123 & $1.378(12)$ \\
\hline C123 & C124 & $1.376(13)$ \\
\hline C124 & $\mathrm{C} 125$ & $1.375(13)$ \\
\hline C125 & C126 & $1.385(11)$ \\
\hline C127 & C128 & $1.406(10)$ \\
\hline C127 & C132 & $1.388(9)$ \\
\hline C128 & C129 & $1.380(10)$ \\
\hline C129 & C130 & $1.391(11)$ \\
\hline C130 & C131 & $1.387(12)$ \\
\hline C131 & C132 & $1.394(10)$ \\
\hline C133 & C134 & $1.383(10)$ \\
\hline C133 & C138 & $1.409(10)$ \\
\hline C134 & C135 & $1.377(11)$ \\
\hline C135 & C136 & $1.399(12)$ \\
\hline C136 & C137 & $1.401(13)$ \\
\hline C137 & C138 & $1.378(12)$ \\
\hline C139 & $\mathrm{C} 140$ & $1.400(10)$ \\
\hline C139 & C144 & $1.377(11)$ \\
\hline C140 & C141 & $1.387(11)$ \\
\hline C141 & C142 & $1.364(13)$ \\
\hline C142 & C143 & $1.386(13)$ \\
\hline C143 & C144 & $1.366(12)$ \\
\hline
\end{tabular}


Table 2: Bond Angles in ${ }^{\circ}$

\begin{tabular}{|c|c|c|c|}
\hline Atom & Atom & Atom & Angle $/^{\circ}$ \\
\hline$\overline{01}$ & $\mathrm{Nd} 1$ & S1 & $25.80(12)$ \\
\hline 01 & $\mathrm{Nd} 1$ & 02 & $51.56(16)$ \\
\hline 02 & $\mathrm{Nd} 1$ & $\mathrm{~S} 1$ & $26.41(11)$ \\
\hline 06 & $\mathrm{Nd} 1$ & $\mathrm{~S} 1$ & $64.23(12)$ \\
\hline 06 & $\mathrm{Nd} 1$ & 01 & $76.15(17)$ \\
\hline 06 & $\mathrm{Nd} 1$ & 02 & $64.69(17)$ \\
\hline 07 & $\mathrm{Nd} 1$ & $\mathrm{~S} 1$ & $130.54(13)$ \\
\hline 07 & $\mathrm{Nd} 1$ & 01 & $121.23(17)$ \\
\hline 07 & $\mathrm{Nd} 1$ & 02 & $138.25(17)$ \\
\hline 07 & $\mathrm{Nd} 1$ & 06 & $73.64(17)$ \\
\hline 07 & $\mathrm{Nd} 1$ & 09 & $150.86(19)$ \\
\hline 07 & $\mathrm{Nd} 1$ & 010 & $81.10(17)$ \\
\hline 07 & $\mathrm{Nd} 1$ & 011 & $76.09(17)$ \\
\hline 08 & $\mathrm{Nd} 1$ & $\mathrm{~S} 1$ & $108.73(13)$ \\
\hline 08 & $\mathrm{Nd} 1$ & 01 & $134.52(17)$ \\
\hline 08 & $\mathrm{Nd} 1$ & 02 & $83.22(17)$ \\
\hline 08 & $\mathrm{Nd} 1$ & 06 & $80.41(18)$ \\
\hline 08 & $\mathrm{Nd} 1$ & 07 & $87.61(18)$ \\
\hline 08 & $\mathrm{Nd} 1$ & 09 & $87.64(18)$ \\
\hline 08 & $\mathrm{Nd} 1$ & 010 & $149.31(18)$ \\
\hline 08 & $\mathrm{Nd} 1$ & 011 & $74.69(16)$ \\
\hline 09 & $\mathrm{Nd} 1$ & $\mathrm{~S} 1$ & $78.00(13)$ \\
\hline 09 & $\mathrm{Nd} 1$ & 01 & $81.32(17)$ \\
\hline 09 & $\mathrm{Nd} 1$ & 02 & $69.44(18)$ \\
\hline 09 & $\mathrm{Nd} 1$ & 06 & $133.57(17)$ \\
\hline 09 & $\mathrm{Nd} 1$ & 010 & $88.55(18)$ \\
\hline 09 & $\mathrm{Nd} 1$ & 011 & $74.92(17)$ \\
\hline 010 & $\mathrm{Nd} 1$ & $\mathrm{~S} 1$ & $100.19(12)$ \\
\hline 010 & $\mathrm{Nd} 1$ & 01 & $74.68(17)$ \\
\hline 010 & $\mathrm{Nd} 1$ & 02 & $123.50(17)$ \\
\hline 010 & $\mathrm{Nd} 1$ & 06 & $122.62(17)$ \\
\hline 010 & $\mathrm{Nd} 1$ & 011 & $74.92(16)$ \\
\hline 011 & $\mathrm{Nd} 1$ & S1 & $152.56(12)$ \\
\hline 011 & $\mathrm{Nd} 1$ & 01 & $141.54(16)$ \\
\hline 011 & $\mathrm{Nd} 1$ & 02 & $138.53(16)$ \\
\hline 011 & $\mathrm{Nd} 1$ & 06 & $141.26(17)$ \\
\hline $\mathrm{S} 2$ & $\mathrm{~S} 1$ & $\mathrm{Nd} 1$ & $106.60(9)$ \\
\hline 01 & $\mathrm{~S} 1$ & $\mathrm{Nd} 1$ & $48.4(2)$ \\
\hline 01 & $\mathrm{~S} 1$ & $\mathrm{~S} 2$ & $106.1(2)$ \\
\hline 02 & $\mathrm{~S} 1$ & $\mathrm{Nd} 1$ & $60.4(3)$ \\
\hline 02 & $\mathrm{~S} 1$ & $\mathrm{~S} 2$ & $106.9(3)$ \\
\hline 02 & $\mathrm{~S} 1$ & 01 & $107.0(4)$ \\
\hline 03 & $\mathrm{~S} 1$ & $\mathrm{Nd} 1$ & $149.0(3)$ \\
\hline 03 & $\mathrm{~S} 1$ & $\mathrm{~S} 2$ & $103.5(3)$ \\
\hline 03 & $\mathrm{~S} 1$ & 01 & $115.5(3)$ \\
\hline 03 & $\mathrm{~S} 1$ & 02 & $116.9(4)$ \\
\hline S1 & $\mathrm{S} 2$ & S3 & $100.04(12)$ \\
\hline 04 & S3 & $\mathrm{S} 2$ & $103.0(3)$ \\
\hline 04 & S3 & 05 & $115.8(4)$ \\
\hline 04 & S3 & 06 & $114.9(3)$ \\
\hline 05 & S3 & $\mathrm{S} 2$ & $106.1(3)$ \\
\hline 05 & S3 & 06 & $112.0(4)$ \\
\hline 06 & S3 & $\mathrm{S} 2$ & $103.3(2)$ \\
\hline 07 & $\mathrm{P} 1$ & $\mathrm{C} 1$ & $109.7(3)$ \\
\hline 07 & $\mathrm{P} 1$ & $\mathrm{C} 7$ & $114.2(3)$ \\
\hline 07 & P1 & C13 & $109.9(3)$ \\
\hline $\mathrm{C} 1$ & P1 & C13 & $109.0(3)$ \\
\hline $\mathrm{C} 7$ & P1 & $\mathrm{C} 1$ & $108.6(3)$ \\
\hline $\mathrm{C} 7$ & P1 & C13 & $105.2(4)$ \\
\hline 08 & P2 & C19 & $113.0(3)$ \\
\hline
\end{tabular}




\begin{tabular}{|c|c|c|c|}
\hline Atom & Atom & Atom & Angle $/{ }^{\circ}$ \\
\hline$\overline{08}$ & P2 & $\mathrm{C} 25$ & $111.6(3)$ \\
\hline 08 & P2 & C31 & $110.2(3)$ \\
\hline C19 & P2 & $\mathrm{C} 25$ & $106.4(3)$ \\
\hline C31 & P2 & C19 & $108.1(3)$ \\
\hline C31 & P2 & $\mathrm{C} 25$ & $107.3(3)$ \\
\hline 09 & P3 & C37 & $110.6(3)$ \\
\hline 09 & P3 & $\mathrm{C} 43$ & $109.9(3)$ \\
\hline 09 & P3 & C49 & $112.8(3)$ \\
\hline C37 & P3 & C49 & $108.0(3)$ \\
\hline $\mathrm{C} 43$ & P3 & C37 & $109.3(3)$ \\
\hline $\mathrm{C} 43$ & P3 & C49 & $106.1(3)$ \\
\hline 010 & $\mathrm{P} 4$ & C55 & $112.0(3)$ \\
\hline 010 & $\mathrm{P} 4$ & C61 & $111.8(3)$ \\
\hline 010 & $\mathrm{P} 4$ & C67 & $111.3(3)$ \\
\hline C55 & $\mathrm{P} 4$ & C61 & $109.0(3)$ \\
\hline $\mathrm{C} 55$ & P4 & C67 & $106.9(3)$ \\
\hline C61 & $\mathrm{P} 4$ & C67 & $105.6(3)$ \\
\hline S1 & 01 & $\mathrm{Nd} 1$ & $105.8(3)$ \\
\hline $\mathrm{S} 1$ & 02 & $\mathrm{Nd} 1$ & $93.2(3)$ \\
\hline S3 & 06 & $\mathrm{Nd} 1$ & $127.4(3)$ \\
\hline $\mathrm{P} 1$ & 07 & $\mathrm{Nd} 1$ & $167.6(3)$ \\
\hline P2 & 08 & $\mathrm{Nd} 1$ & $169.9(3)$ \\
\hline P3 & 09 & $\mathrm{Nd} 1$ & $166.9(3)$ \\
\hline $\mathrm{P} 4$ & 010 & $\mathrm{Nd} 1$ & $170.3(3)$ \\
\hline $\mathrm{C} 2$ & $\mathrm{C} 1$ & $\mathrm{P} 1$ & $118.3(6)$ \\
\hline $\mathrm{C} 2$ & $\mathrm{C} 1$ & $\mathrm{C} 6$ & $120.2(7)$ \\
\hline C6 & $\mathrm{C} 1$ & $\mathrm{P} 1$ & $121.3(6)$ \\
\hline $\mathrm{C} 1$ & $\mathrm{C} 2$ & C3 & $119.0(7)$ \\
\hline $\mathrm{C} 4$ & $\mathrm{C} 3$ & $\mathrm{C} 2$ & $120.9(8)$ \\
\hline $\mathrm{C} 5$ & $\mathrm{C} 4$ & $\mathrm{C} 3$ & $120.2(8)$ \\
\hline $\mathrm{C} 4$ & $\mathrm{C} 5$ & $\mathrm{C} 6$ & $121.0(8)$ \\
\hline $\mathrm{C} 1$ & C6 & $\mathrm{C} 5$ & $118.8(7)$ \\
\hline $\mathrm{C} 8$ & $\mathrm{C} 7$ & $\mathrm{P} 1$ & $123.6(6)$ \\
\hline $\mathrm{C} 8$ & $\mathrm{C} 7$ & $\mathrm{C} 12$ & $118.8(7)$ \\
\hline $\mathrm{C} 12$ & $\mathrm{C} 7$ & $\mathrm{P} 1$ & $117.6(5)$ \\
\hline $\mathrm{C} 7$ & C8 & C9 & $121.5(7)$ \\
\hline $\mathrm{C} 8$ & $\mathrm{C} 9$ & C10 & $119.0(7)$ \\
\hline C11 & $\mathrm{C} 10$ & $\mathrm{C} 9$ & $119.8(7)$ \\
\hline $\mathrm{C} 12$ & C11 & C10 & $120.8(7)$ \\
\hline C11 & $\mathrm{C} 12$ & $\mathrm{C} 7$ & $120.0(7)$ \\
\hline C14 & C13 & $\mathrm{P} 1$ & $121.3(6)$ \\
\hline C18 & C13 & P1 & $119.6(6)$ \\
\hline C18 & C13 & C14 & 119.1(7) \\
\hline C15 & C14 & C13 & $120.1(7)$ \\
\hline C16 & C15 & C14 & $119.5(8)$ \\
\hline C17 & C16 & C15 & $120.7(8)$ \\
\hline C16 & C17 & C18 & $120.2(7)$ \\
\hline C13 & C18 & C17 & $120.4(7)$ \\
\hline $\mathrm{C} 20$ & C19 & $\mathrm{P} 2$ & $121.9(5)$ \\
\hline $\mathrm{C} 20$ & C19 & $\mathrm{C} 24$ & $120.1(6)$ \\
\hline $\mathrm{C} 24$ & C19 & $\mathrm{P} 2$ & $118.0(5)$ \\
\hline $\mathrm{C} 21$ & $\mathrm{C} 20$ & C19 & $120.2(8)$ \\
\hline $\mathrm{C} 20$ & $\mathrm{C} 21$ & $\mathrm{C} 22$ & $119.4(8)$ \\
\hline $\mathrm{C} 23$ & $\mathrm{C} 22$ & $\mathrm{C} 21$ & $120.5(7)$ \\
\hline $\mathrm{C} 22$ & $\mathrm{C} 23$ & $\mathrm{C} 24$ & $120.3(8)$ \\
\hline $\mathrm{C} 23$ & $\mathrm{C} 24$ & C19 & $119.5(7)$ \\
\hline $\mathrm{C} 26$ & $\mathrm{C} 25$ & $\mathrm{P} 2$ & $121.8(5)$ \\
\hline $\mathrm{C} 26$ & $\mathrm{C} 25$ & C30 & $119.5(7)$ \\
\hline C30 & $\mathrm{C} 25$ & $\mathrm{P} 2$ & $118.7(5)$ \\
\hline $\mathrm{C} 27$ & $\mathrm{C} 26$ & $\mathrm{C} 25$ & $119.6(6)$ \\
\hline $\mathrm{C} 26$ & $\mathrm{C} 27$ & $\mathrm{C} 28$ & $120.3(7)$ \\
\hline $\mathrm{C} 29$ & $\mathrm{C} 28$ & $\mathrm{C} 27$ & $120.0(7)$ \\
\hline
\end{tabular}




\begin{tabular}{|c|c|c|c|}
\hline Atom & Atom & Atom & Angle $/^{\circ}$ \\
\hline$\overline{\mathrm{C} 28}$ & $\mathrm{C} 29$ & C30 & $119.8(7)$ \\
\hline $\mathrm{C} 29$ & $\mathrm{C} 30$ & $\mathrm{C} 25$ & $120.9(7)$ \\
\hline C32 & C31 & $\mathrm{P} 2$ & $121.3(6)$ \\
\hline C36 & C31 & $\mathrm{P} 2$ & $118.1(5)$ \\
\hline C36 & C31 & C32 & $120.6(7)$ \\
\hline C33 & $\mathrm{C} 32$ & C31 & $120.1(7)$ \\
\hline C32 & $\mathrm{C} 33$ & C34 & $118.9(7)$ \\
\hline C35 & C34 & C33 & $121.2(7)$ \\
\hline C34 & C35 & C36 & $119.2(7)$ \\
\hline C31 & C36 & C35 & $119.9(7)$ \\
\hline C38 & C37 & P3 & $118.1(6)$ \\
\hline $\mathrm{C} 42$ & C37 & P3 & $122.3(6)$ \\
\hline $\mathrm{C} 42$ & $\mathrm{C} 37$ & C38 & $119.5(7)$ \\
\hline C39 & $\mathrm{C} 38$ & C37 & $119.5(7)$ \\
\hline $\mathrm{C} 40$ & C39 & $\mathrm{C} 38$ & $120.5(8)$ \\
\hline C39 & $\mathrm{C} 40$ & $\mathrm{C} 41$ & $120.6(8)$ \\
\hline $\mathrm{C} 40$ & $\mathrm{C} 41$ & $\mathrm{C} 42$ & $120.1(8)$ \\
\hline C37 & $\mathrm{C} 42$ & $\mathrm{C} 41$ & $119.8(8)$ \\
\hline $\mathrm{C} 44$ & $\mathrm{C} 43$ & P3 & $119.0(6)$ \\
\hline $\mathrm{C} 44$ & $\mathrm{C} 43$ & C48 & $119.6(7)$ \\
\hline $\mathrm{C} 48$ & $\mathrm{C} 43$ & P3 & $121.3(6)$ \\
\hline $\mathrm{C} 43$ & $\mathrm{C} 44$ & $\mathrm{C} 45$ & $120.6(7)$ \\
\hline $\mathrm{C} 46$ & $\mathrm{C} 45$ & $\mathrm{C} 44$ & $119.8(7)$ \\
\hline $\mathrm{C} 45$ & $\mathrm{C} 46$ & $\mathrm{C} 47$ & $119.7(7)$ \\
\hline $\mathrm{C} 48$ & $\mathrm{C} 47$ & $\mathrm{C} 46$ & $120.4(8)$ \\
\hline $\mathrm{C} 47$ & $\mathrm{C} 48$ & $\mathrm{C} 43$ & $119.8(7)$ \\
\hline $\mathrm{C} 50$ & $\mathrm{C} 49$ & P3 & $116.7(5)$ \\
\hline C54 & $\mathrm{C} 49$ & P3 & $122.9(6)$ \\
\hline C54 & $\mathrm{C} 49$ & C50 & $120.3(7)$ \\
\hline C51 & $\mathrm{C} 50$ & C49 & $119.4(7)$ \\
\hline C52 & C51 & C50 & $119.8(7)$ \\
\hline C51 & $\mathrm{C} 52$ & C53 & 121.1(7) \\
\hline C54 & C53 & C52 & $118.7(7)$ \\
\hline C49 & C54 & C53 & $120.6(7)$ \\
\hline $\mathrm{C} 56$ & C55 & $\mathrm{P} 4$ & $118.2(5)$ \\
\hline C56 & C55 & C60 & $120.0(6)$ \\
\hline C60 & C55 & $\mathrm{P} 4$ & $121.8(5)$ \\
\hline $\mathrm{C} 57$ & C56 & C55 & $119.5(7)$ \\
\hline C56 & C57 & C58 & $120.6(7)$ \\
\hline C59 & C58 & C57 & $119.9(7)$ \\
\hline C58 & C59 & $\mathrm{C} 60$ & $120.7(7)$ \\
\hline C59 & $\mathrm{C} 60$ & C55 & $119.3(7)$ \\
\hline $\mathrm{C} 62$ & C61 & $\mathrm{P} 4$ & $121.8(5)$ \\
\hline C66 & C61 & $\mathrm{P} 4$ & $118.7(5)$ \\
\hline C66 & C61 & C62 & $119.5(6)$ \\
\hline C63 & $\mathrm{C} 62$ & C61 & $120.5(7)$ \\
\hline C64 & C63 & C62 & $119.3(7)$ \\
\hline $\mathrm{C} 65$ & C64 & C63 & $121.0(7)$ \\
\hline C64 & $\mathrm{C} 65$ & C66 & $120.0(7)$ \\
\hline C61 & C66 & C65 & $119.6(7)$ \\
\hline C68 & C67 & $\mathrm{P} 4$ & $121.7(5)$ \\
\hline $\mathrm{C} 72$ & $\mathrm{C} 67$ & $\mathrm{P} 4$ & $119.1(5)$ \\
\hline $\mathrm{C} 72$ & C67 & C68 & 119.1(7) \\
\hline C69 & C68 & C67 & $120.4(7)$ \\
\hline C68 & C69 & $\mathrm{C} 70$ & $119.3(7)$ \\
\hline C71 & $\mathrm{C} 70$ & C69 & $121.2(7)$ \\
\hline $\mathrm{C} 70$ & C71 & $\mathrm{C} 72$ & $119.0(7)$ \\
\hline $\mathrm{C} 67$ & $\mathrm{C} 72$ & C71 & $120.9(7)$ \\
\hline 012 & $\mathrm{Nd} 2$ & S6 & $64.98(17)$ \\
\hline 012 & $\mathrm{Nd} 2$ & 016 & $74.9(3)$ \\
\hline 012 & $\mathrm{Nd} 2$ & 017 & $67.3(2)$ \\
\hline 012 & $\mathrm{Nd} 2$ & 022 & $144.4(2)$ \\
\hline
\end{tabular}




\begin{tabular}{|c|c|c|c|}
\hline Atom & Atom & Atom & Angle $/^{\circ}$ \\
\hline$\overline{016}$ & $\mathrm{Nd} 2$ & S6 & $25.58(15)$ \\
\hline 016 & $\mathrm{Nd} 2$ & 017 & $52.0(2)$ \\
\hline 016 & $\mathrm{Nd} 2$ & 022 & $137.4(2)$ \\
\hline 017 & $\mathrm{Nd} 2$ & S6 & $26.97(15)$ \\
\hline 018 & $\mathrm{Nd} 2$ & S6 & $79.72(14)$ \\
\hline 018 & $\mathrm{Nd} 2$ & 012 & $136.8(2)$ \\
\hline 018 & $\mathrm{Nd} 2$ & 016 & $84.0(2)$ \\
\hline 018 & $\mathrm{Nd} 2$ & 017 & $70.0(2)$ \\
\hline 018 & $\mathrm{Nd} 2$ & 020 & $146.80(19)$ \\
\hline 018 & $\mathrm{Nd} 2$ & 021 & $88.75(18)$ \\
\hline 018 & $\mathrm{Nd} 2$ & 022 & $72.71(17)$ \\
\hline 019 & $\mathrm{Nd} 2$ & S6 & $96.39(14)$ \\
\hline 019 & $\mathrm{Nd} 2$ & 012 & $117.6(3)$ \\
\hline 019 & $\mathrm{Nd} 2$ & 016 & $71.2(2)$ \\
\hline 019 & $\mathrm{Nd} 2$ & 017 & $120.2(2)$ \\
\hline 019 & $\mathrm{Nd} 2$ & 018 & $89.1(2)$ \\
\hline 019 & $\mathrm{Nd} 2$ & 020 & $81.40(19)$ \\
\hline 019 & $\mathrm{Nd} 2$ & 021 & $152.5(2)$ \\
\hline 019 & $\mathrm{Nd} 2$ & 022 & $73.3(2)$ \\
\hline 020 & $\mathrm{Nd} 2$ & S6 & $132.76(13)$ \\
\hline 020 & $\mathrm{Nd} 2$ & 012 & $74.4(2)$ \\
\hline 020 & $\mathrm{Nd} 2$ & 016 & $121.9(2)$ \\
\hline 020 & $\mathrm{Nd} 2$ & 017 & $141.46(19)$ \\
\hline 020 & $\mathrm{Nd} 2$ & 022 & $74.09(17)$ \\
\hline 021 & $\mathrm{Nd} 2$ & S6 & $110.15(14)$ \\
\hline 021 & $\mathrm{Nd} 2$ & 012 & $81.4(3)$ \\
\hline 021 & $\mathrm{Nd} 2$ & 016 & $135.7(2)$ \\
\hline 021 & $\mathrm{Nd} 2$ & 017 & $84.5(2)$ \\
\hline 021 & $\mathrm{Nd} 2$ & 020 & $85.51(18)$ \\
\hline 021 & $\mathrm{Nd} 2$ & 022 & $80.0(2)$ \\
\hline 022 & $\mathrm{Nd} 2$ & S6 & $150.50(11)$ \\
\hline 022 & $\mathrm{Nd} 2$ & 017 & $139.7(2)$ \\
\hline 012 & $\mathrm{~S} 4$ & S5 & $107.3(3)$ \\
\hline 013 & S4 & S5 & $102.5(5)$ \\
\hline 013 & S4 & 012 & $108.9(8)$ \\
\hline 014 & $\mathrm{~S} 4$ & S5 & $110.2(8)$ \\
\hline 014 & S4 & 012 & $112.3(8)$ \\
\hline 014 & S4 & 013 & $114.8(10)$ \\
\hline S6 & S5 & S4 & $101.89(16)$ \\
\hline S5 & S6 & $\mathrm{Nd} 2$ & $108.56(12)$ \\
\hline 015 & S6 & $\mathrm{Nd} 2$ & $147.3(3)$ \\
\hline 015 & S6 & S5 & $103.9(3)$ \\
\hline 015 & S6 & 016 & $116.9(4)$ \\
\hline 015 & S6 & 017 & $117.4(4)$ \\
\hline 016 & S6 & $\mathrm{Nd} 2$ & $49.0(3)$ \\
\hline 016 & S6 & S5 & $108.3(3)$ \\
\hline 016 & S6 & 017 & $102.8(4)$ \\
\hline 017 & S6 & $\mathrm{Nd} 2$ & $55.4(3)$ \\
\hline 017 & S6 & S5 & $107.0(3)$ \\
\hline 018 & P5 & C73 & $109.6(3)$ \\
\hline 018 & P5 & C79 & $113.0(3)$ \\
\hline 018 & P5 & C85 & $110.8(3)$ \\
\hline C73 & P5 & C79 & $108.1(3)$ \\
\hline C73 & P5 & C85 & $109.0(3)$ \\
\hline C79 & P5 & C85 & $106.2(3)$ \\
\hline 019 & P6 & C91 & $111.3(3)$ \\
\hline 019 & P6 & $\mathrm{C} 97$ & $110.9(4)$ \\
\hline 019 & P6 & C103 & $111.0(3)$ \\
\hline C91 & P6 & C103 & $110.3(3)$ \\
\hline
\end{tabular}




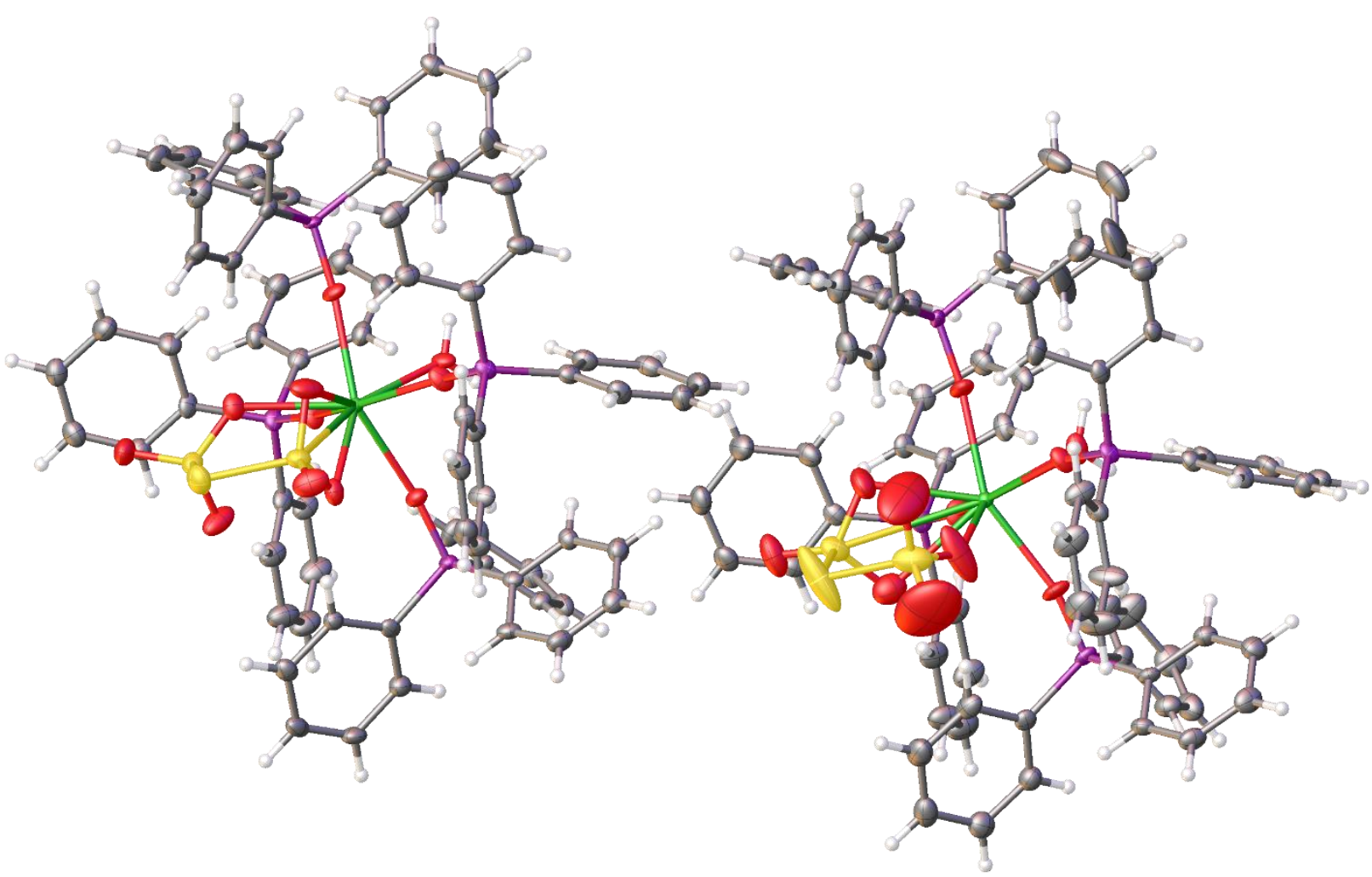

\section{Citations}

CrysAlisPro Software System, Rigaku Oxford Diffraction, (2019).

O.V. Dolomanov and L.J. Bourhis and R.J. Gildea and J.A.K. Howard and H. Puschmann, Olex2: A complete structure solution, refinement and analysis program, J. Appl. Cryst., (2009), 42, 339-341.

Sheldrick, G.M., Crystal structure refinement with ShelXL, Acta Cryst., (2015), C27, 3-8.

Sheldrick, G.M., ShelXT-Integrated space-group and crystal-structure determination, Acta Cryst., (2015), A71, 3-8. 